\title{
Weighted Essentially Non- Oscillatory Simulations and Modeling of the Complex Hydrodynamic Flows - Part 1. Regular Shock Refraction
}

\author{
O. Schilling (LLNL) and M. Latini (CalTech)
}

\section{April 30, 2004}




\section{DISCLAIMER}

This document was prepared as an account of work sponsored by an agency of the United States Government. Neither the United States Government nor the University of California nor any of their employees, makes any warranty, express or implied, or assumes any legal liability or responsibility for the accuracy, completeness, or usefulness of any information, apparatus, product, or process disclosed, or represents that its use would not infringe privately owned rights. Reference herein to any specific commercial product, process, or service by trade name, trademark, manufacturer, or otherwise, does not necessarily constitute or imply its endorsement, recommendation, or favoring by the United States Government or the University of California. The views and opinions of authors expressed herein do not necessarily state or reflect those of the United States Government or the University of California, and shall not be used for advertising or product endorsement purposes.

Work performed under the auspices of the U.S. Department of Energy by Lawrence Livermore National Laboratory under Contract W-7405-ENG-48. 


\title{
Weighted Essentially Non-Oscillatory Simulations and Modeling of Complex Hydrodynamic Flows. Part 1. Regular Shock Refraction
}

\author{
Marco Latini* $\quad$ Oleg Schilling ${ }^{\dagger}$
}

April 30, 2004

\begin{abstract}
Shock refraction is a fundamental shock phenomenon observed when shocks interact with a material interface separating gases with different properties. Following refraction, a transmitted shock enters the second gas and a reflected wave returns back into the first gas. In the case of regular shock refraction all waves meet at a single point called the triplepoint, creating five different states for the two gases. Analytical methods based on shock polar analysis $[9,16]$ have been developed to determine the state of two ideal gases in each of the five refraction regions. Furthermore, shock refraction constitutes a basic example of complex hydrodynamic flows. For this reason, shock refraction is used in this report as one validation of the high-order accurate weighted essentially non-oscillatory (WENO) shock-capturing method, as implemented in the HOPE code. The following two-step validation process is adopted. First, analytical results are obtained for the normal and oblique shock refraction (with shock-interface angle $\beta_{i n t}=75^{\circ}$ ) observed for a $M a=1.2$ shock. To validate the single-fluid and the two-fluid implementations of the WENO method, two pairs of gases, argon/xenon, having equal adiabatic exponents $\gamma$ and air(acetone)/sulfur hexafluoride, having different adiabatic exponents $\gamma$, are considered. Both the light-toheavy and heavy-to-light configurations are considered. Second, numerical simulations are performed using the fifth-order WENO method and values of the density, pressure, temperature, speed of sound, and flow velocity in each of the five refraction regions are compared with the analytical predictions from shock polar analysis. In all cases considered, excellent agreement between the simulation results and the analytical predictions was found. The results from this investigation suggest that the WENO method is a very useful numerical method for the simulation and modeling of complex hydrodynamic flows.
\end{abstract}

\footnotetext{
${ }^{*}$ Applied and Computational Mathematics, California Institute of Technology, Pasadena, CA 91125. E-mail: mlatini@caltech.edu

${ }^{\dagger}$ University of California, Lawrence Livermore National Laboratory, Livermore, CA 94551. E-mail: schilling1@llnl.gov
} 


\section{Contents}

1 Introduction $\quad 5$

2 Review of shock relations and refraction theory $\quad 6$

2.1 Review of the normal and oblique shock relations for an ideal gas . . . . . . . 6

2.1.1 The normal shock relations . . . . . . . . . . . . . 7

2.1.2 The oblique shock relations . . . . . . . . . . . . . 7

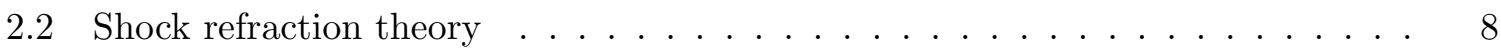

2.2.1 Normal shock refraction . . . . . . . . . . . . . . . . . 11

2.2 .2 Oblique shock refraction . . . . . . . . . . . . . . 15

3 The weighted essentially non-oscillatory (WENO) method 19

3.1 Overview of the WENO method implementation for the Euler equations . . . . 19

3.1.1 The two-dimensional Euler equations of gas dynamics . . . . . . . . 19

3.1.2 Predecessor of the flux-averaged WENO method: the ENO method and the cell-averaged WENO method . . . . . . . . . . . . . . . . 21

3.1.3 The flux-averaged WENO method . . . . . . . . . . . . . . . . 24

3.1.4 The third-order TVD Runge-Kutta time-evolution scheme . . . . . . . 25

3.1.5 Summary of algorithm . . . . . . . . . . . . . . . 25

3.2 The (High-Order Polynomial Expansion) HOPE code . . . . . . . . . . 27

3.2 .1 Overview of the HOPE code . . . . . . . . . . . . . . . 27

3.2 .2 Features of the HOPE code . . . . . . . . . . . . . . . 28

4 Numerical simulations of shock refraction $\quad 31$

4.1 Initial conditions . . . . . . . . . . . . . . . . . . . . . . 31

4.2 Boundary Conditions . . . . . . . . . . . . . . . . . . 33

4.3 Refraction for gases with the same adiabatic exponent: the case of argon and

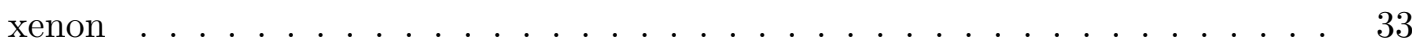

4.3.1 Normal shock refraction at an Ar-Xe interface with a reflected shock . . 33

4.3.2 Normal shock refraction at an Xe-Ar interface with a reflected rarefaction wave ............................ 34

4.3.3 Oblique shock refraction at an Ar-Xe interface with a reflected shock . . 38

4.3.4 Oblique shock refraction at an Xe-Ar interface with a reflected rarefaction wave . . . . . . . . . . . . . . . . 42

4.4 Refraction with different adiabatic exponents: the case of air(acetone) and $\mathrm{SF}_{6} \quad 51$

4.4.1 Normal shock refraction at an air(acetone)-SF $\mathrm{SF}_{6}$ interface with a reflected shock wave . . . . . . . . . . . . . . . . 5 51

4.4.2 Normal shock refraction at an $\mathrm{SF}_{6}$-air(acetone) interface with a reflected rarefaction wave ..................... 51

4.4.3 Oblique shock refraction at an air(acetone)-SF $\mathrm{SF}_{6}$ interface with a reflected shock ........................... 51

4.4.4 Oblique shock refraction at an $\mathrm{SF}_{6}$-air(acetone) interface with a reflected rarefaction wave ....................... 59

5 Conclusions $\quad 65$ 


\section{List of Figures}

1 Shock adiabat for air . . . . . . . . . . . . . . . . . 9

2 Flow across an oblique shock . . . . . . . . . . . . . . . . 9

3 The flow turning angle $\beta$ and shock angle $\beta$ in air . . . . . . . . . . . . . . 10

4 Shock polar for air . . . . . . . . . . . . . . . . . . . 10

$5 \quad$ Normal shock refraction in $(x, t)$-plane I . . . . . . . . . . . . . . 13

6 Normal shock refraction in $(x, t)$-plane II . . . . . . . . . . . . . . . 13

7 Normal shock waves in $(u, p)$-plane . . . . . . . . . . . . . 16

8 Rarefaction waves in $(u, p)$-plane . . . . . . . . . . . . . . 16

9 Oblique shock refraction in $(x, y)$-plane I . . . . . . . . . . . . . . 20

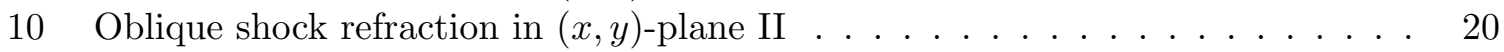

11 Ar-Xe normal shock refraction in $(u, p)$-plane . . . . . . . . . . . . . . . . 35

12 WENO simulation of Ar-Xe normal shock refraction . . . . . . . . . . 36

13 Xe-Ar normal shock refraction in $(u, p)$-plane . . . . . . . . . . . . . 39

14 WENO simulation of Xe-Ar normal shock refraction . . . . . . . . . . . . 40

15 Ar-Xe oblique shock refraction in $(\theta, p)$-plane . . . . . . . . . . . . . . 43

16 Ar-Xe oblique shock refraction in $(\theta, p)$-plane (close up) . . . . . . . . . . . 43

17 WENO simulation of Ar-Xe oblique shock refraction . . . . . . . . . . . 44

18 Xe-Ar oblique shock refraction in $(\theta, p)$-plane . . . . . . . . . . . . . . . 47

19 Xe-Ar oblique shock refraction in $(\theta, p)$-plane (close up) . . . . . . . . . . 47

20 WENO simulation of Xe-Ar oblique shock refraction . . . . . . . . . . . 49

21 air(acetone)-SF 6 normal shock refraction in $(u, p)$-plane . . . . . . . . . . 52

22 WENO simulation of air(acetone)-SF $\mathrm{SF}_{6}$ normal shock refraction . . . . . . . . 53

$23 \mathrm{SF}_{6}$-air(acetone) normal shock refraction in $(u, p)$-plane $\ldots \ldots \ldots \ldots$

24 WENO simulation of $\mathrm{SF}_{6}$-air(acetone) normal shock refraction $\ldots \ldots \ldots$

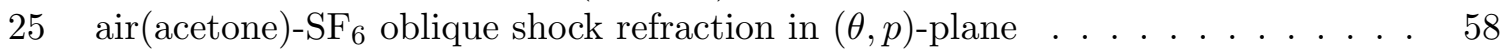

26 air(acetone)-SF 6 oblique shock refraction in $(\theta, p)$-plane (close up) . . . . . . 58

27 WENO simulation of air(acetone)-SF $\mathrm{SF}_{6}$ oblique shock refraction . . . . . . . . 61

$28 \mathrm{SF}_{6}$-air(acetone) oblique shock refraction in $(\theta, p)$-plane . . . . . . . . . . 62

$29 \mathrm{SF}_{6}$-air(acetone) oblique shock refraction in $(\theta, p)$-plane (close up) . . . . . . 62

30 WENO simulation of $\mathrm{SF}_{6}$-air(acetone) oblique shock refraction $\ldots \ldots$. . . . . 63

\section{List of Tables}

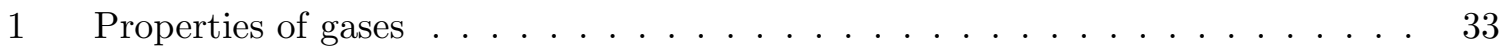

2 Shock Mach numbers for normal Ar-Xe refraction . . . . . . . . . . . . . . . . . . . . . . . 35

3 Results for Ar-Xe normal shock refraction . . . . . . . . . . . . . . . . 37

4 Shock Mach numbers for normal Xe-Ar refraction . . . . . . . . . . . . . . 39

5 Results for Xe-Ar normal shock refraction . . . . . . . . . . . . . . . . 41

6 Shock Mach numbers for oblique Ar-Xe refraction . . . . . . . . . . . . . . 42

7 Results for Ar-Xe oblique shock refraction . . . . . . . . . . . . . . . . 45

8 Shock Mach numbers for oblique Xe-Ar shock refraction . . . . . . . . . . . 46

9 Mach numbers of flow ahead of and behind reflected rarefaction wave in oblique

Xe-Ar shock refraction . . . . . . . . . . . . . . . . 46

10 Results for Xe-Ar oblique shock refraction . . . . . . . . . . . . . . 50

11 Shock Mach numbers for normal air(acetone)-SF ${ }_{6}$ refraction . . . . . . . . . . . 52 
12 Results for air(acetone) $-\mathrm{SF}_{6}$ normal shock refraction . . . . . . . . . . . 54

13 Shock Mach numbers for $\mathrm{SF}_{6}$-air(acetone) refraction . . . . . . . . . . . 55

14 Results for $\mathrm{SF}_{6}$-air(acetone) normal shock refraction . . . . . . . . . . . 57

15 Shock Mach numbers for oblique air(acetone)- $\mathrm{SF}_{6}$ shock refraction . . . . . . . 59

16 Results for air(acetone)-SF 6 oblique shock refraction . . . . . . . . . . . . 60

17 Shock Mach numbers for $\mathrm{SF}_{6}$-air(acetone) oblique shock refraction . . . . . . . 61

18 Mach numbers of flows ahead of and behind reflected rarefaction wave in oblique

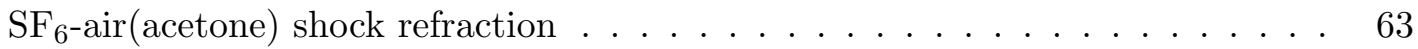

19 Results for $\mathrm{SF}_{6}$-air(acetone) oblique shock refraction . . . . . . . . . . . 64 


\section{Introduction}

Shock refraction is observed when shocks interact with a material interface separating gases with different properties. Following the interaction, a transmitted shock enters the second gas and a reflected wave returns back into the first gas. The reflected wave can be either a rarefaction wave, a Mach wave, or a shock wave. The material interface can be oriented normal to the direction of shock propagation or inclined at an angle $\beta$. When the interface is inclined at an angle, the incident shock, the transmitted shock and the reflected rarefaction waves all coalesce at a single point-the triple-point. Such a configuration is referred to as regular shock refraction. Note that regular shock refraction always occurs in the case of normal shocks as a triple-point is observed in the $(x, t)$-plane. However, in the case of oblique shock refraction, for certain Mach numbers and angles $\beta$ it is found that the waves no longer meet at a single point: this is referred to as irregular shock refraction. The challenge of shock refraction is understanding and modeling the complex wave structures and predicting the state of the gases in the different refraction regions. In the case of regular shock refraction, five refraction regions are observed and shock polar analysis has been used to analytically determine the state of the gas $[9,16]$.

In the present work, regular shock refraction is proposed and adopted as a validation for shock-capturing methods, and in particular, the weighted essentially non-oscillatory (WENO) method-a modern reconstruction-evolution method currently being used in a variety of applications, including shock-induced mixing and supersonic turbulence. The choice of this flow configuration is largely motivated by current work on the Richtmyer-Meshkov instability observed when an oblique perturbed interface interacts with a shock [25]. First, two pairs of gases having equal adiabatic exponents $\gamma$ are considered, argon and xenon, to validate the single-fluid implementation of the WENO method; then, an air-acetone mixture and $\mathrm{SF}_{6}$ having different adiabatic exponents is considered to validate the two-fluid ('gamma-blending') implementation. Two configurations are considered for each pair of gases, the light-to-heavy and the heavy-to-light cases, to validate the method in the presence of a reflected shock wave and a reflected rarefaction wave. Next, both the case of a normal and of an oblique shock refraction are considered to validate the code in the presence of geometric changes. Thus, a total of eight different configurations are considered in the present investigation. For each configuration, analytical predictions based on shock polar analysis for the density, pressure, speed of sound, temperature, and flow velocity are obtained in each of the five refraction regions. Next, numerical simulations are performed and values from each of the refraction regions are compared with the analytical predictions. Excellent agreement between the numerical simulation results and analytical predictions is observed in all eight cases. The results lend confidence to the numerical implementation of the WENO method in the HOPE code, and indicate that this method (code) is very well-suited for the investigation of complex hydrodynamics flows generated by shocks interacting with material interfaces. Furthermore, the investigation suggests that shock refraction constitutes a suitable complex hydrodynamic validation for which exact analytical solutions are available. Subsequent parts of this report describe numerical simulations, analysis, and modeling of shock-induced mixing in various flow configurations, including the single-mode Richtmyer-Meshkov instability with reshock (Part 2 [24]), the oblique single-mode Richtmyer-Meshkov instability (Part 3 [25]), and the three-dimensional multimode Richtmyer-Meshkov instability (Part 4 [26]).

The organization of this report is as follows. Presented in $\S 2$ is a review of the normal and oblique shock relations, followed by the theory of shock refraction based on shock polar 
analysis used to determine the states of the gases in each of the five refraction regions. The WENO method and details of the HOPE code used in this investigation are presented in $\S 3$. Although presently used for the simulation of two-dimensional shock refraction, HOPE provides a framework for the multi-dimensional numerical simulation of the fully-nonlinear evolution of hydrodynamic instabilities and late-time mixing. Finally, in $\S 4$ refraction theory is applied to the eight cases described above, numerical simulation results corresponding to these cases are presented, and comparisons from the five refraction regions are made to the analytical predictions.

\section{Review of shock relations and refraction theory}

The basic properties of shocks and the shock jump conditions needed for the subsequent analysis are reviewed in this section. Next, shock refraction theory is developed based on shock jump conditions and matching the pressure and normal velocity across material interfaces. Results from the theory are applied to specific problems and compared with numerical simulations in $\S 4$.

\subsection{Review of the normal and oblique shock relations for an ideal gas}

Shocks can be regarded as thin regions of rapid state variations, and can therefore be idealized as discontinuity surfaces of essentially zero thickness moving through space. All thermodynamic properties such as temperature, density, pressure, and velocity experience a 'jump' across a shock. The jump in properties must satisfy the normal and oblique shock relations, which provide a statement of the conservation laws for mass, momentum, and energy. These relations are obtained when conservation laws are applied in a control volume around a shock, and have extensive applications in the analysis of shock dynamics and in the construction of numerical methods for hyperbolic conservation laws admitting discontinuous solutions (see $[27])$.

Let the subscripts 1 and 2 denote the state ahead of and behind the shock, respectively. Let brackets denote the difference of a quantity $f$ across the shock: $[f] \equiv f_{2}-f_{1}$. Then the shock jump conditions express the conservation of mass, momentum, and energy as [28]

$$
\begin{aligned}
{[\rho u] } & =0 \\
{\left[\rho u^{2}+p\right] } & =0 \\
{\left[h+\frac{u^{2}}{2}\right] } & =0 \\
{[s] } & >0
\end{aligned}
$$

where $\rho$ is the density, $u$ is the flow velocity, $p$ is the pressure, $h=U+p / \rho$ is the enthalpy ( $U$ is the internal energy), and $s$ is the entropy. Note that Eq. (3) expresses total enthalpy conservation across a shock (so that a shock is adiabatic). The last equation expresses the fact that entropy increases across a shock; this is expected as a shock can be regarded as an instantaneous, irreversible change. Note that the entropy condition shows that only compression shocks with $[p]>0$ can be observed.

Combining the above conservation equations to eliminate the velocity yields the RankineHugoniot relation

$$
[h]=\frac{V_{1}+V_{2}}{2}[p],
$$


where $V_{i}=1 / \rho_{i}$ is the specific volume of fluid $i$. Note that this equation contains only thermodynamic quantities. If the upstream conditions are known and the enthalpy can be computed from the equation of state, then a functional relation $p_{2}=p\left(V_{2}\right)$ can be obtained. This relation represents a curve in the $(p, V)$-plane (the adiabat) and determines the possible final states given the initial states $\left(p_{1}, V_{1}\right)$. For an ideal gas, the adiabat is given by

$$
\frac{p_{2}}{p_{1}}=\frac{\frac{\gamma+1}{\gamma-1}-\frac{V_{2}}{V_{1}}}{\frac{\gamma+1}{\gamma-1} \frac{V_{2}}{V_{1}}-1},
$$

where the adiabatic exponent (ratio of specific heats) $\gamma=c_{p} / c_{v}$ is the ratio of the specific heats at constant pressure $c_{p}$ and constant volume $c_{v}$. Figure 1 shows a plot of the adiabat for air.

Let

$$
\Pi \equiv \frac{[p]}{\rho_{1} c_{s, 1}^{2}}
$$

be the non-dimensional pressure jump (where $c_{s, 1}$ is the sound speed in fluid 1), which measures the strength of a shock. A shock is weak if $\Pi \ll 1$ and is strong if $\Pi \gg 1$.

\subsubsection{The normal shock relations}

For an ideal gas, the dimensionless pressure jump $\Pi$ can be expressed as functions of the shock Mach number $M a_{1}$ and the adiabatic exponent $\gamma$ thus yielding

$$
\begin{aligned}
\Pi & =\frac{2}{\gamma+1}\left(M a_{1}^{2}-1\right) \\
\frac{[p]}{p_{1}} & =\gamma \Pi, \\
-\frac{[u]}{c_{s, 1}} & =\frac{\Pi}{M a_{1}}, \\
-\frac{[V]}{V_{1}} & =\frac{\Pi}{M a_{1}^{2}}, \\
\frac{[T]}{T_{1}} & =(\gamma-1) \frac{1+\frac{\gamma}{2} \Pi}{1+\frac{\gamma+1}{2} \Pi} \Pi, \\
M a_{2}^{2} & =\frac{M a_{1}^{2}+\frac{2}{\gamma-1}}{\gamma-1} M a_{1}^{2}-1 \\
\frac{[s]}{R_{g}} & =\ln \left\{\left[1+\frac{2 \gamma}{\gamma+1}\left(M a_{1}^{2}-1\right)\right]^{1 /(\gamma-1)}\left[\frac{(\gamma+1) M a_{1}^{2}}{(\gamma-1) M a_{1}^{2}+1}\right]^{-\gamma /(\gamma-1)}\right\}
\end{aligned}
$$

\subsubsection{The oblique shock relations}

Oblique shocks can be considered as normal shocks observed in a reference frame moving together with the shock, as illustrated in Fig. 2. An oblique shock is characterized by the shock angle $\beta$, which measures the inclination of the shock with respect to the velocity $\mathbf{U}_{1}$, where $\mathbf{U}_{1}=\left(u_{1}, v_{1}\right)$ denotes the velocity ahead of the shock. Applying the normal shock relations to $u_{1}=\left\|\mathbf{U}_{1}\right\| \sin \beta$, the component of the velocity field normal to the shock, yields $u_{2}$. Note that in order to observe a shock, $u_{1}$ must be supersonic. Thus, $M a_{1} \sin \beta>1$ or

$$
\beta>\mu=\sin ^{-1}\left(\frac{1}{M a_{1}}\right),
$$


where $\mu$ is the Mach angle. As the tangential components are equal, $v_{2}=v_{1}=\left\|U_{1}\right\| \cos \beta$ and $u_{2}<u_{1}$, it follows that an oblique shock rotates the flow by an angle $\theta$. Geometrical considerations show that $\tan \beta=u_{1} / v_{1}$ and $\tan (\beta-\theta)=u_{2} / v_{1}$, which yields the relation

$$
-\frac{[u]}{v_{1}}=\frac{1}{\cos ^{2} \beta(\cot \theta+\tan \beta)} .
$$

The above relations can be further simplified for an ideal gas. In particular, the flow turning angle $\theta=\theta\left(\beta, M a_{1}\right)$ can be determined by

$$
\tan \theta=\frac{\cot \beta}{\frac{\gamma+1}{2}\left(\sin ^{2} \beta-\frac{1}{M a_{1}^{2}}\right)^{-1}-1} .
$$

Note that for any $\theta$ there are two possible shock angles $\beta$, as shown in Fig. 3. The smaller and larger $\beta$ correspond to the weaker and stronger shock, respectively. In practice, the weaker shock corresponding to the smaller $\beta$ is observed when downstream conditions permit. See [40] for additional information on this topic.

Similarly, the change in pressure $\Delta p$ ahead of and behind the shock can be plotted as a function of the flow turning angle $\theta$. This yields the shock polar shown in Fig. 4. This form of the shock polar is very important for the oblique shock refraction, as will be shown later. As expected, the point of maximum change in pressure corresponds to the normal shock.

\subsection{Shock refraction theory}

Shock refraction is observed whenever a shock interacts with a material contact surface, and is a fundamental shock phenomenon that has been extensively investigated. The first theoretical work on shock refraction is due to Taub [39]. Taub used the Rankine-Hugoniot conditions to enforce the continuity of pressure and of the normal component of the velocity across the interface, which yields an algebraic system for the tangent of the deflection angle with five independent parameters. Taub was first to realize that this system of equations admits multiple solutions, and that no solutions exist for certain configurations. Polachek and Seeger [32] further investigated the equations and conducted a numerical survey of the parameters to determine which solutions are physically observed and under what conditions the solutions do not exist. When solutions do not exist, irregular shock refraction is observed: this case was first investigated by Henderson [16]. Henderson was also the first to introduce shock polar analysis in the investigation of shock refraction phenomena.

The first experimental work on planar shock refraction is the work of Jahn [12], who conducted shock tube experiments using air- $\mathrm{CH}_{4}$ and air- $\mathrm{CO}_{2}$. Jahn compared the angles of refraction with the theoretical prediction of Polachek and Seeger [32] and found excellent agreement. Henderson and collaborators subsequently conducted extensive experimental, theoretical, and numerical studies for $\mathrm{CO}_{2}-\mathrm{He}$, air- $\mathrm{SF}_{6}$, and $\mathrm{CO}_{2}-\mathrm{CH}_{4}$ to elucidate the complex hydrodynamics and wave behavior of shock refraction (see [15, 16, 18, 17, 19, 2, 1, 30]). More recently, Samtaney and Pullin [33] numerically investigated shock refraction at an interface with shock Mach numbers larger than 10.

Henderson broadly classifies shock refraction as 'fast-slow' and 'slow-fast' depending on whether the speed of the wave in the incident medium $U_{i}$ is lesser or greater than the speed of the transmitted shock $U_{t}$. Let

$$
n=\frac{\left|U_{i}\right|}{\left|U_{t}\right|}
$$




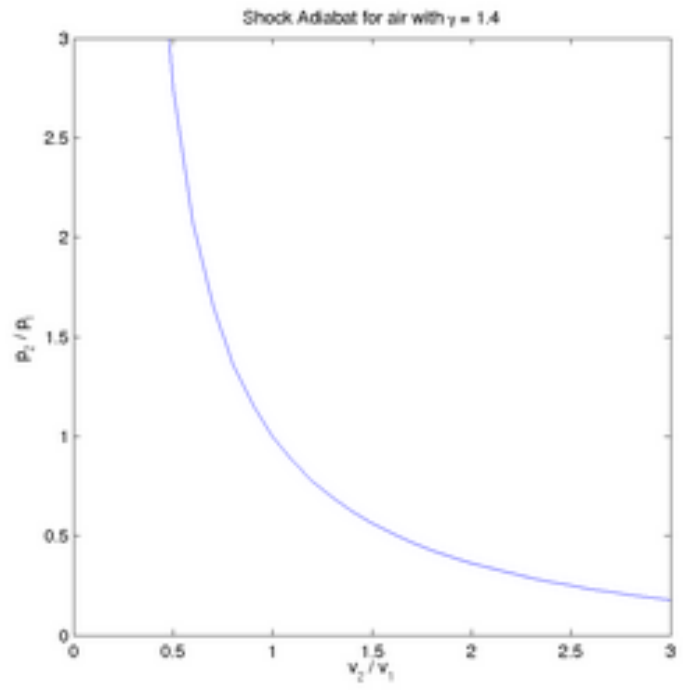

Figure 1: The shock adiabat for air with $\gamma=1.4$ in the $(V, p)$-plane. Each point represents a state, and a shock can only connect states on the adiabat.

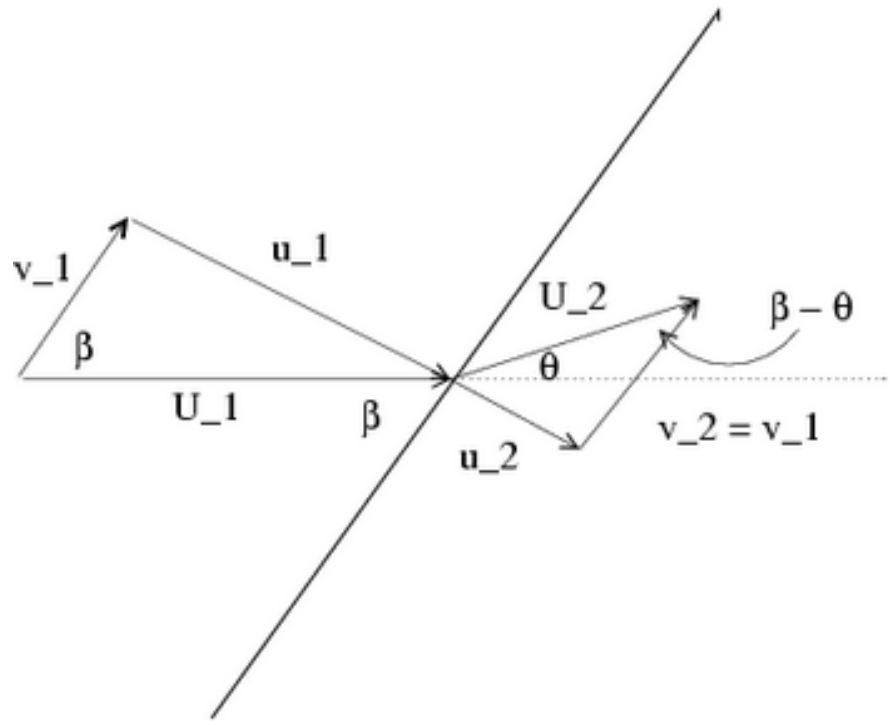

Figure 2: The flow across an oblique shock. Note the oblique shock angle $\beta$ and the flow turning angle $\theta$. 


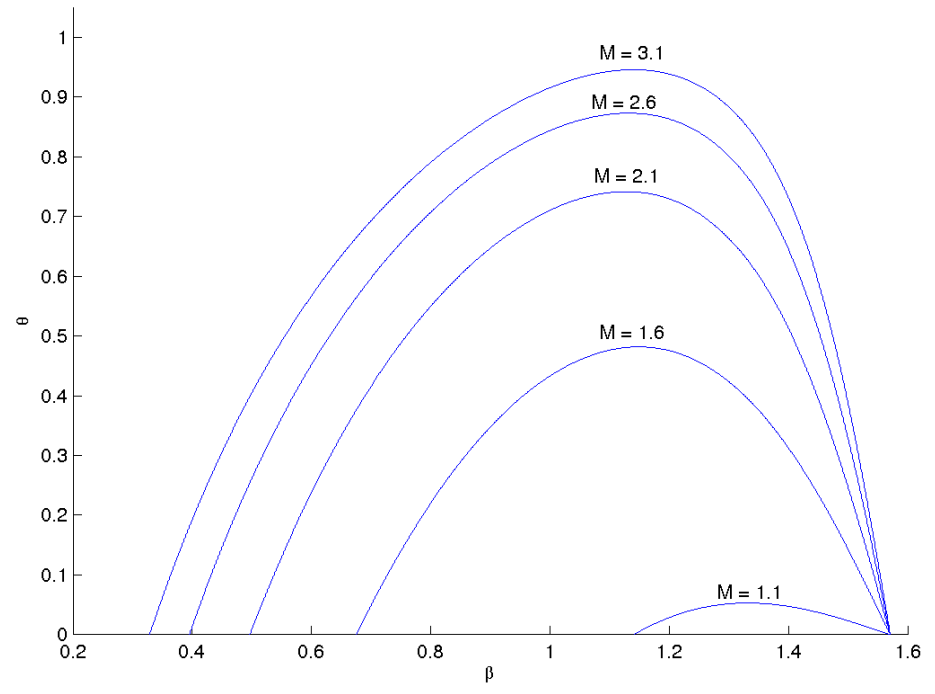

Figure 3: The flow turning angle $\theta$ as a function of the shock angle $\beta$ at different Mach numbers for oblique shocks in air with $\gamma=1.4$. The shock angle varies between $\beta_{\text {min }}=\sin ^{-1}\left(1 / M_{s}\right)$ and $\beta_{\max }=\pi / 2$. As the shock Mach number increases, $\beta_{\min }$ decreases and $\theta_{\max }$ increases. Also note that, in general, for every $\theta$ there are two possible shock angles $\beta$.

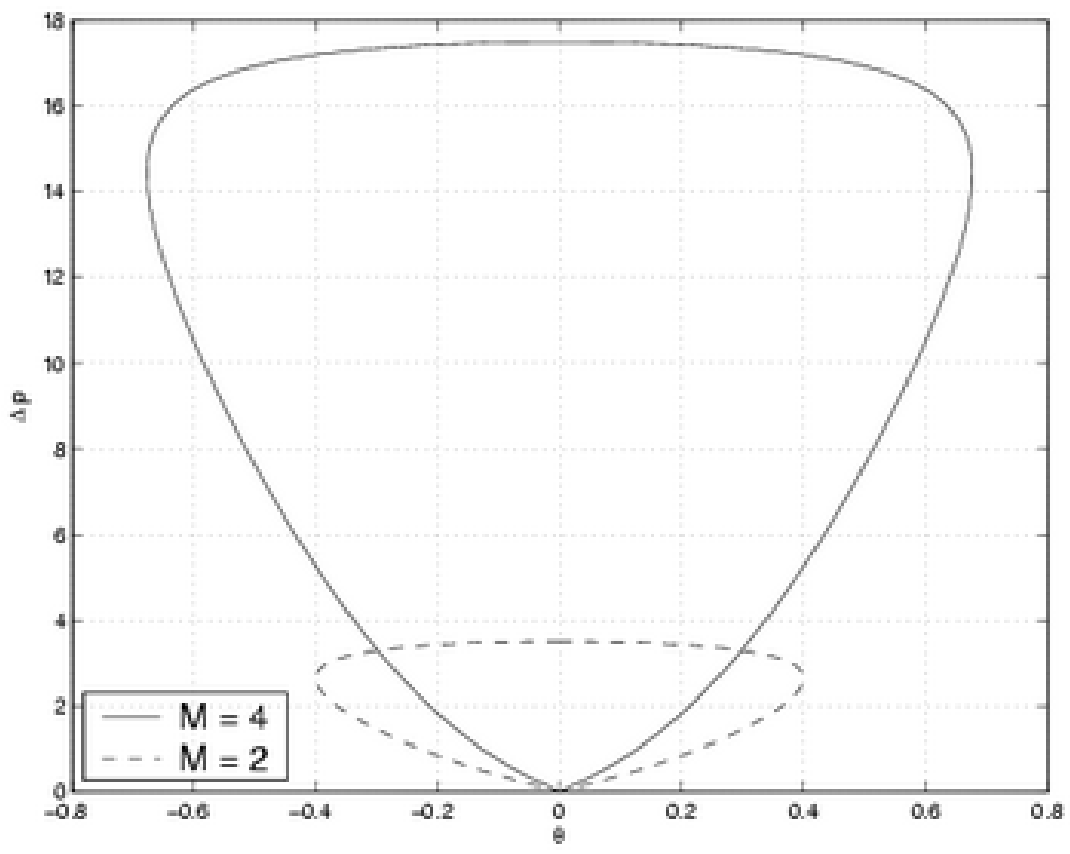

Figure 4: The shock polar in the $(\Delta p, \theta)$-plane for air with $\gamma=1.4$ for $M a=2$ and $M a=4$. 
denote the refractive index. If $n<1$ the refraction is slow-fast, and a reflected rarefaction is observed; if $n>1$ the refraction is fast-slow, and a reflected shock wave is observed; and if $n=1$ a reflected Mach wave is observed, corresponding to the case of total transmission. 'Slowfast' refraction usually occurs when the shock refracts from a heavier gas (as measured by the density) into a lighter gas; 'fast-slow' when the shock refracts from a lighter gas into a heavier gas. For this reason the 'slow-fast' and 'fast-slow' refractions are ofter called 'heavy-to-light' and 'light-to-heavy' respectively. Although this latter naming convention can be inaccurate as shown by Henderson [17] it has been extensively used in the literature and is adopted in the present report. A second broad classification is regular versus irregular refraction. This classification applies to refractions when the interface and the incident wave form an angle $\beta$. The refraction is regular if the incident shock, the transmitted shock, and the reflected wave all coincide at a triple-point. Otherwise, the refraction is irregular.

In regular refraction it is possible to obtain a reflected shock or a reflected rarefaction wave. To predict which type of reflected wave is observed for a given flow configuration, Henderson used shock polar diagrams. Shock polar diagrams represent the flow turning angle $\theta$ and the change in pressure $\Delta p$ observed behind an oblique shock at a given Mach number as a function of the shock inclination angle $\beta$. These diagrams provide an intuitive graphical method to determine the solution of the refraction problem (see $[34,35]$ ). This method also reveals that non-unique solutions are possible in most cases. Henderson claims that the weakest solution is usually observed in experiments and in practice, where the 'weakest solution' is the solution that induces the least change in pressure.

In this section it is shown how analytical results based on the shock jump (RankineHugoniot) conditions and matching normal velocity and pressure across material interfaces can be used to predict the angle and the flow conditions in each of the refraction regions. First, the unsteady normal shock refraction is considered, and then the steady oblique shock refraction problem is considered. The theory developed here is applied in $\S 4$ to specific examples, and the results are compared with those from numerical simulations.

\subsubsection{Normal shock refraction}

Consider normal shock refraction observed when a normal shock is incident on a contact surface separating two ideal gases with different densities, adiabatic exponents, or temperatures. The pressure and the normal velocities must be the same on both sides of the contact surface. The contact surface is modeled as a surface of discontinuity having zero thickness. Let the subscripts 1 and 2 denote the gas ahead of the incident shock and to the left of the contact surface, and the gas to the right of the contact surface, respectively. When a normal shock is incident on a contact surface, the shock refracts. Following refraction, a shock is transmitted into the second gas and a reflected wave is observed returning back into the first gas. The reflected wave can be a shock, as shown in Fig. 5 or a reflected rarefaction wave, as shown in Fig. 6. During the transition from a reflected shock to a reflected rarefaction wave, a reflected Mach wave returns back into the first gas. This corresponds to total transmission. It is of interest to predict the nature of the reflected wave and the states following refraction.

Figures 5 and 6 illustrate the refraction process observed in the $(x, t)$-plane. In this plane the $x$-axis represent the spatial position and the $y$-axis represents time. Thus, the initially stationary contact surface is a straight vertical line, while the moving shock is represented by an oblique line. The $(x, t)$-plane allows a simultaneous view of the entire refraction process. Such $x-t$ diagrams are extensively used in the theoretical, numerical, and experimental investigation 
of shock phenomena.

The following convention is used for the regions of the shock refraction throughout this work. Denote by region 1 the state ahead of the initial shock in the first gas and by region 2 the state on the other side of the contact surface in the second gas. Region 3 denotes the state behind the initial incident shock in the first gas, and region 4 denotes the state behind the transmitted shock in the second gas. Finally, region 5 denotes the state behind the reflected wave in the first gas. The motivation for this convention is that across material interfaces the numbers always differ by one, and behind waves the numbers always differ by two. Consequently, the first and second gas contains states with odd and even numbers, respectively.

An elegant method to analyze the refraction problem is due to Courant and Friedrichs [9], who were the first to consider shock dynamics in the $(u, p)$-plane. The $(u, p)$-plane is a natural choice for shock refraction, as the pressure and velocity must match across a contact surface. Therefore, in the $(u, p)$-plane, the states in region 1 and region 2 are represented by the same point and similarly, regions 4 and 5 are represented by the same point. The general theory is developed as follows. Denote the states with subscripts $a$ and $b$. Suppose that the initial state $\left(u_{a}, p_{a}\right)$ is given. Then the jump conditions for shocks and rarefaction waves provide a definite set of possible finite states $\left(u_{b}, p_{b}\right)$. If the initial state is represented as a point in the $(u, p)$-plane, the final states after a shock or a rarefaction wave lie on certain curves. These curves can be easily determined as follows.

In the case of shocks, the Hugoniot relation

$$
\frac{p_{b}}{p_{a}}=\frac{\rho_{b}-\lambda \rho_{a}}{\rho_{b}-\lambda \rho_{a}}
$$

where $\lambda=(\gamma-1) /(\gamma+1)$, combined with the other shock jump conditions yields

$$
\frac{p_{b}-p_{a}}{u_{b}-u_{a}}= \pm \sqrt{\frac{p_{b}+\lambda p_{a}}{(1-\lambda) v_{a}}} .
$$

From this, the velocity $u_{b}$ can be expressed as a function of the final pressure $p_{b}$ and the known quantities in region 1:

$$
u_{b}=u_{a} \pm \phi_{a}\left(p_{b}\right)
$$

where

$$
\phi_{a}(p)=\left(p-p_{a}\right) \sqrt{\frac{(1-\lambda) v_{a}}{p+\lambda p_{a}}} .
$$

The plus or minus sign is used for shocks moving to the right or left, respectively.

For completeness, consider all of the possible states that can be connected to region $a$ via shocks. For a:

1. right-moving shock from region 1 into region $2, p_{a}>p_{b}$ and $u_{a}>u_{b}$ as the shock has already passed in region $a$;

2. left-moving shock from region $b$ into region $a, p_{b}>p_{a}$; furthermore, when the shock moves to the left, $u_{b}<u_{a}$ as a flow initially at rest has $u_{a}=0$ and the flow moves to the left with $u_{b}<0$ later;

3. right-moving shock from region $b$ into region $a, p_{b}>p_{a}$ and $u_{b}>u_{a}$ as region $b$ has already interacted with the shock, and; 


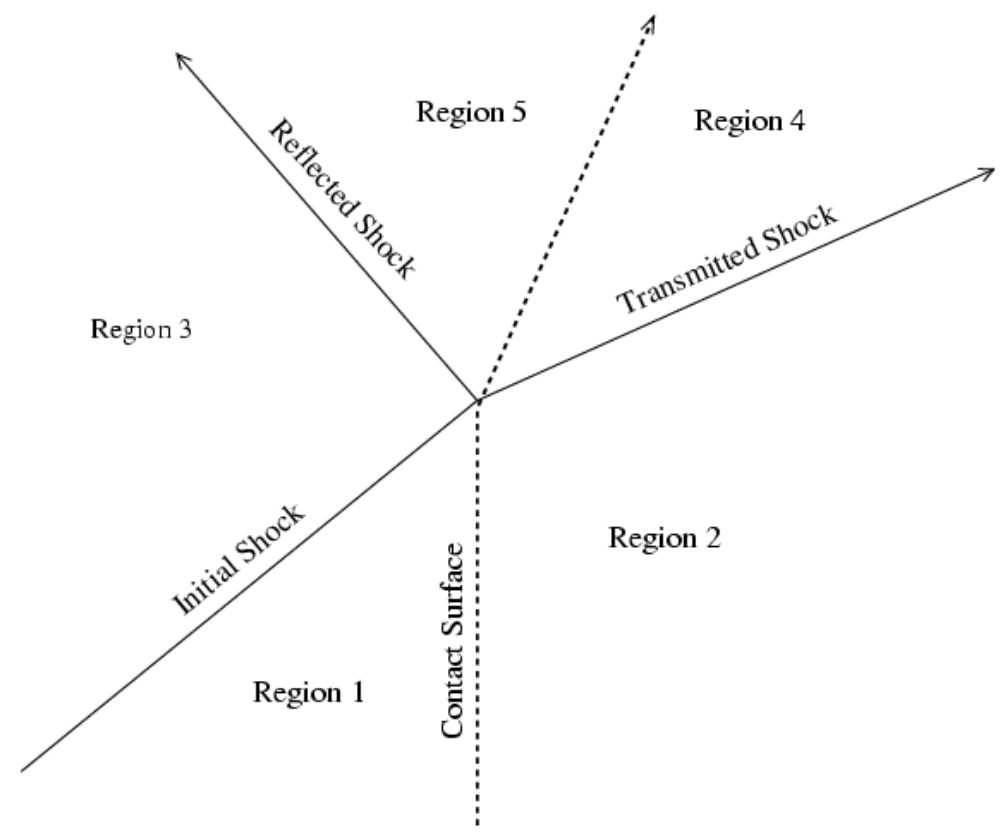

Figure 5: A normal shock wave interacting with a contact surface in the $(x, t)$-plane. Note the reflected and the transmitted shocks.

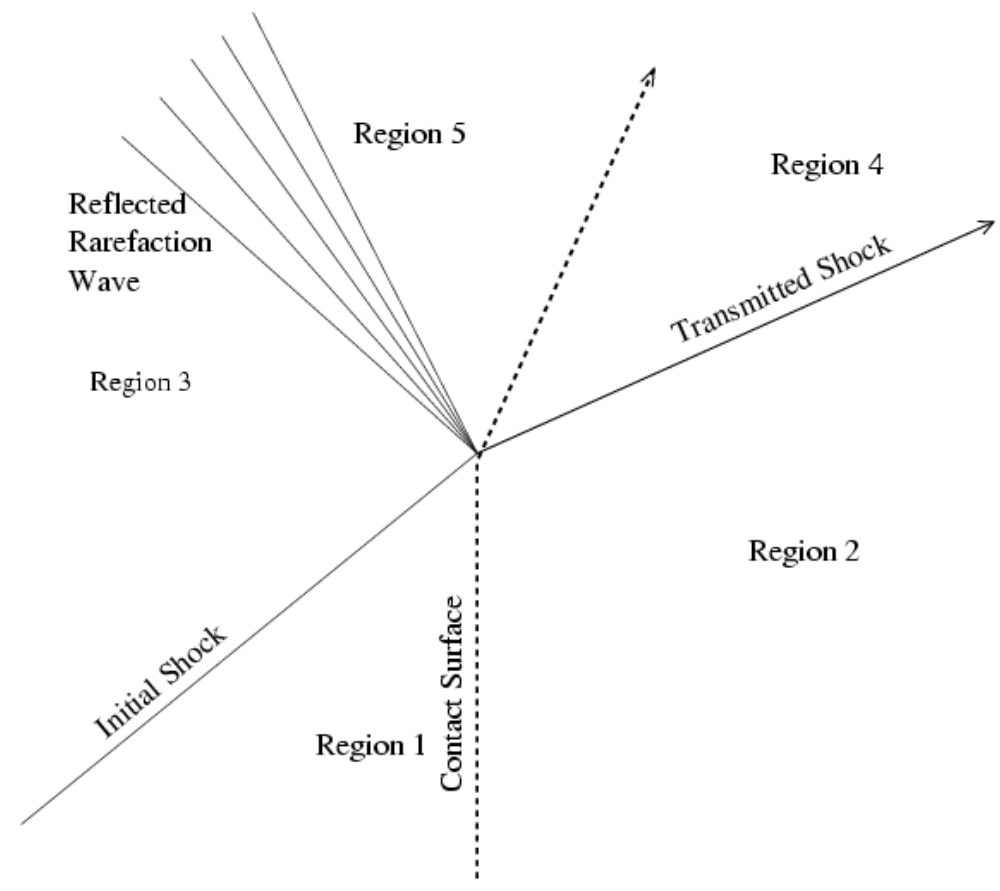

Figure 6: A normal shock wave interacting with a contact surface in the $(x, t)$-plane. Note that the reflected wave is a rarefaction wave. 
4. left-moving shock from region $a$ into region $b, p_{a}>p_{b}$ and $u_{a}<u_{b}$ as a flow initially at rest with $u_{b}=0$ moves to the left with $u_{a}<0$ after the passage of the shock.

The above relations represent all of the states that can be connected via a shock moving to the right or to the left, away or toward the initial point $\left(u_{a}, p_{a}\right)$ in the $(u, p)$-plane. The set of all such states is illustrated in Fig. 7.

Now perform a similar analysis for the rarefaction wave. The objective is to solve for the velocity $u_{b}$ as a function of the pressure $p_{b}$ and of quantities in the initial state $a$. Using Riemann invariants and the adiabatic relations gives

$$
u_{b}=u_{a} \pm \psi_{a}\left(p_{b}\right)
$$

with

$$
\psi_{a}(p) \equiv \frac{\sqrt{1-\lambda^{2}}}{\lambda} \sqrt{v_{a}} p_{a}^{1 /(2 \gamma)}\left[p^{(\gamma-1) /(2 \gamma)}-p_{a}^{(\gamma-1) /(2 \gamma)}\right],
$$

where the plus or minus sign refers to a right-moving or left-moving rarefaction wave, respectively.

A similar analysis can be performed for all states connected by a rarefaction wave. For a:

1. right-moving rarefaction wave from region $a$ into region $b, p_{b}>p_{a}$ and $u_{b}>u_{a}$; a rightmoving rarefaction wave accelerates the flow to the left and a particle initially at rest in region $b$ with $u_{b}=0$ has $u_{a}<0$;

2. left-moving rarefaction wave from region $b$ into region $a, p_{b}<p_{a}$ and $u_{b}>u_{a}$ as the left-moving rarefaction wave accelerates the flow in region $a$ to the right;

3. right-moving rarefaction wave from region $b$ into region $a, p_{b}<p_{a}$ and as the rarefaction wave is moving to the right, the flow is accelerated to the left so that $u_{b}<u_{a}$, and;

4. left-moving rarefaction wave from region $a$ into region $b, p_{a}<p_{b}$ and as the rarefaction accelerates the flow in region $b$ to the right, $u_{b}<u_{a}$.

The above relations represent all of the states that can be connected via a rarefaction wave moving to the right or to the left, away or toward the initial point $\left(u_{a}, p_{a}\right)$ in the $(u, p)$-plane. The set of all such states is illustrated in Fig. 8.

The above curves in the $(u, p)$-plane suggest a method for solving the normal shock rarefaction problem. Consider the initial state $u_{1}=u_{2}$ and $p_{1}=p_{2}$ as the starting point in the $(u, p)$-plane. From the knowledge of the Mach number of the incident shock $M a_{i}$ it is possible to determine the $\left(u_{3}, p_{3}\right)$ location. However, for completeness the entire curve is represented in the $(u, p)$-plane corresponding to a right-moving branch 3 shock originating at $\left(u_{1}, p_{1}\right)$. Then the branch corresponding to case 3 of a right-moving transmitted shock originating at $\left(u_{1}, p_{1}\right)$ is represented. It is a case 3 branch as a right-moving shock is observed from region 4 toward region 2. In general, the branch of the transmitted shock is different from the case 3 branch of the incident shock connecting $\left(u_{1}, p_{1}\right)$ and $\left(u_{3}, p_{3}\right)$. The final state $\left(u_{4}, p_{4}\right)=\left(u_{5}, p_{5}\right)$ is determined by the intersection of the transmitted shock branch with the left-moving rarefaction wave or left-moving shock wave from region $\left(u_{5}, p_{5}\right)$ toward region $\left(u_{3}, p_{3}\right)$. In the terminology developed in the previous section, this corresponds to a left-moving branch 2 rarefaction wave or left-moving branch 2 shock wave originating at $\left(u_{3}, p_{3}\right)$. Note that the two branches for the transmitted shock and the reflected wave move in different directions and, thus, intersect. 
The above analysis also indicates that, if the transmitted shock branch lies above the initial shock branch then, the point $\left(u_{5}, p_{5}\right)$ is obtained by the intersection with the reflected shock wave. However, if the transmitted shock branch lies below the initial shock branch, then the point $\left(u_{5}, p_{5}\right)$ is obtained by the intersection with the reflected rarefaction wave.

This method for solving normal shock refraction is illustrated in $\S 4$ with examples of reflected shock waves and rarefaction waves. Data obtained from numerical simulations using the WENO method are compared with the analytical predictions: this serves as an important validation test case of the WENO method and its implementation in the HOPE code.

\subsubsection{Oblique shock refraction}

Oblique shock refraction is observed when an oblique shock is incident onto an inclined interface or a normal shock incident on an oblique interface. Let $\beta$ denote the angle between the direction of shock propagation and the interface, so that $\beta=90^{\circ}$ corresponds to normal shock refraction and $\beta \neq 90^{\circ}$ corresponds to oblique shock refraction. Specifically, the representative case of $\beta=75^{\circ}$ is considered here. In the oblique shock refraction problem, the system of equations includes the flow turning angle $\theta$ and the shock angle $\beta$. This problem also admits multiple solutions.

When an oblique shock is incident on a contact surface, the shock refracts. Following refraction, a transmitted shock is observed entering the second gas and a reflected wave returns back into the first gas. The contact surface is also deformed by the refraction process. The reflected wave can be either a shock wave as in Fig. 9 or a rarefaction wave as in Fig. 10. During the transition from a reflected shock to a reflected rarefaction, a reflected Mach wave may also be observed, in which case total transmission occurs. Note that in all of the above situations the system of waves meet at a single point, the triple-point. This is referred to as regular refraction. However, for particular angles and conditions, the system of reflected and transmitted waves becomes more complex, and irregular refraction is observed. While regular refraction can be analytically investigated, irregular refraction is much more difficult to study. Thus, the present investigation is limited to regular refraction. For a discussion of irregular refraction, see the work of Henderson [15, 16, 18, 17, 19] and the work with Abd-El-Fattah $[2,1,30]$.

An intuitive method to solve the oblique shock refraction problem is to consider shock polars in the $(\theta, p)$-plane, where $\theta$ is the flow turning angle. This approach was first pioneered by Henderson [16] and additional references can be found in [5] and [6]. The $(\theta, p)$-plane is a natural choice when the regular refraction process is considered in a reference frame moving with the triple-point. In fact, the flows before and after refraction are oriented in the same direction and have the same pressure. Note that in this new frame, the system of shocks is stationary and the initial flow at rest in the laboratory reference frame now moves at the same velocity and in the same direction as the shock. These flows interact with the incident and transmitted shocks at an angle $\beta_{i}$ and $\beta_{t}$, respectively. Following the shock interactions, the flows are turned by the angles $\theta_{i}$ and $\theta_{t}$. These angles can be easily determined using the oblique shock relations. Usually $\theta_{i} \neq \theta_{t}$, and a second wave is needed to turn the flow in the first material by a further angle $\theta_{r}$. Following this second reflected wave, the two flows again move in the same direction. This implies the relation

$$
\theta_{i}+\theta_{r}=\theta_{t}
$$

between the flow turning angles. This relation further emphasizes that the $(\theta, p)$-plane is 


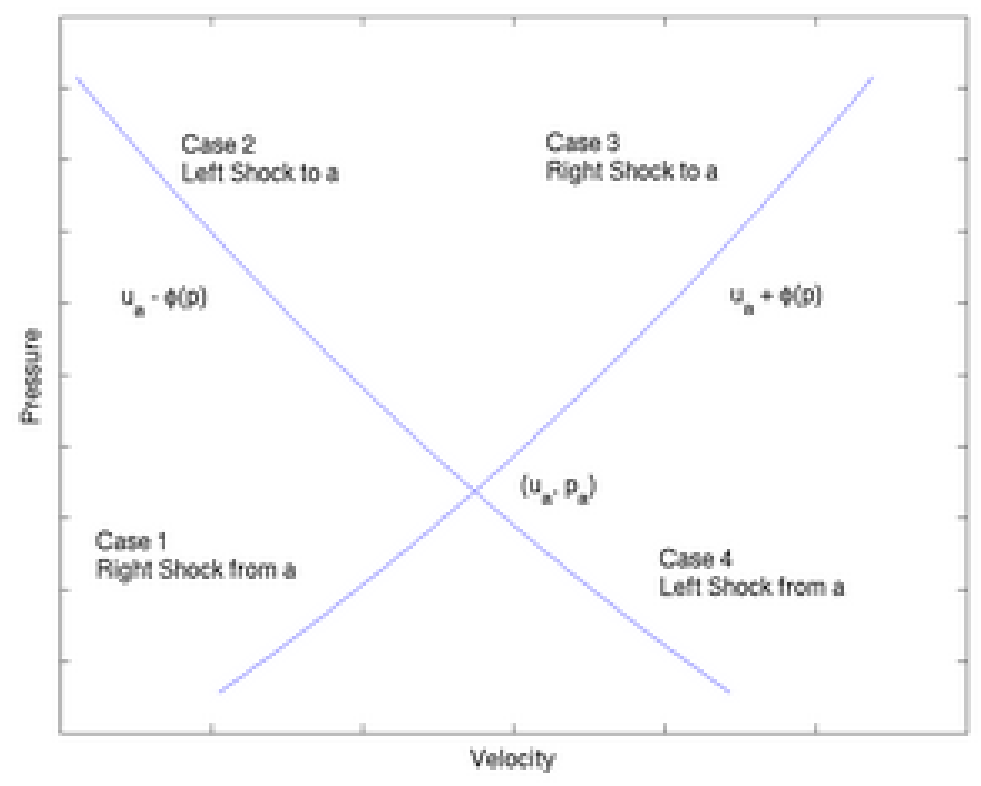

Figure 7: All states in the $(u, p)$-plane that can be reached from the initial $\left(u_{a}, p_{a}\right)$ state with right and left moving shocks.

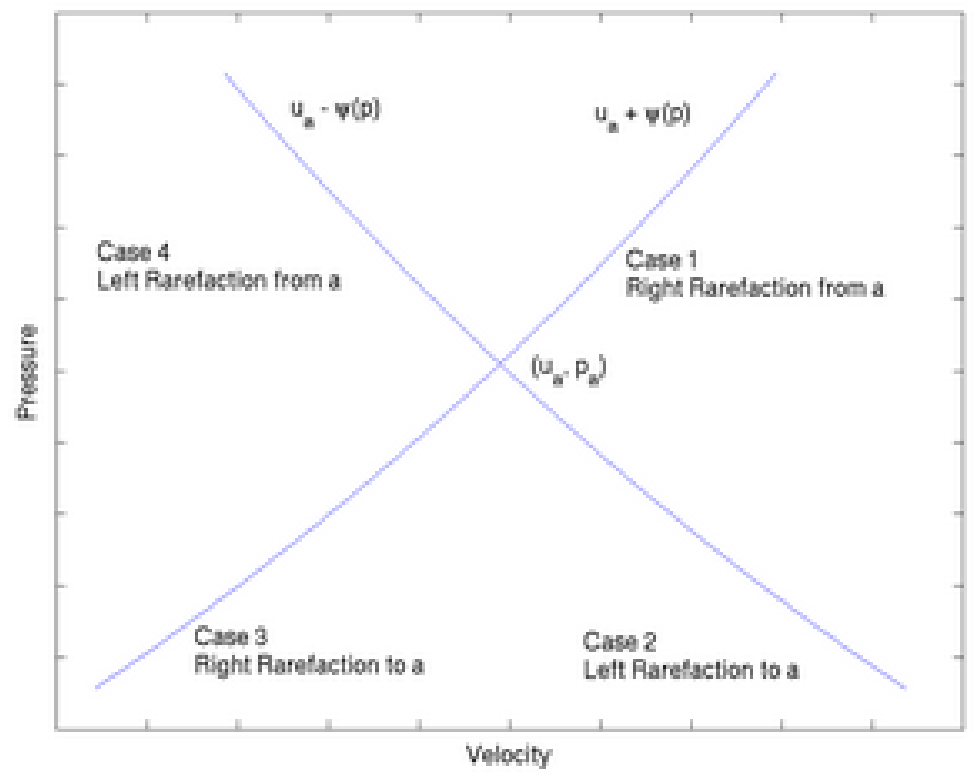

Figure 8: All states in the $(u, p)$-plane that can be reached by the initial $\left(u_{a}, p_{a}\right)$ state via right- and left-moving rarefaction waves. 
the natural choice for the oblique shock refraction problem. In fact, in this plane region 1 and region 2 are represented by the same point and similarly, region 4 and region 5 are also represented by the same point.

The equations for the shock polar can be expressed in many different forms. The form that is most useful for the oblique shock refraction is the shock polar parametrized by the shock angle $\beta$ for a fixed Mach number $M a$. With this parametrization, the reflected shock or reflected rarefaction wave and the transmitted shock 'adjust' their angles $\beta_{r}$ and $\beta_{t}$, respectively, in order to match the pressure and the flow turning angles $\theta_{r}$ and $\theta_{t}$ behind them. Note that the shock angle $\beta$ varies between $\mu$ and $\pi-\mu$ where $\mu=\sin ^{-1}(1 / M a)$ is the Mach angle and $M a$ is the shock Mach number corresponding to the shock polar.

Consider the incident shock in the frame moving with the triple-point. In this reference frame, the flow in region 1 is incident on the shock at a speed $M a_{i}$, and the flow in region 2 is also moving at the same speed. Therefore, the Mach number of the flow in region 2 is

$$
M a_{t}=\frac{c_{s, 1}}{c_{s, 2}} M a_{i}
$$

where $c_{s, 1}$ and $c_{s, 2}$ are the speeds of sound in region 1 and region 2 , respectively. Using $M a_{t}$, the shock polar for the transmitted shock can be represented in the $(\theta, p)$-plane. Similarly, the Mach number $M a_{r}$ of the flow in region 3 can be determined by computing the velocities following the incident shock. Let $u_{3 n}$ be the normal component of the velocity following the shock and let $u_{1 t}$ denote the tangential component of the velocity. Recall that the tangential component of the velocity is the same in region 1 and region 3. Then the Mach number of the flow in region 3 is given by

$$
M a_{r}=\frac{\sqrt{u_{3 n}^{2}+u_{1 t}^{2}}}{c_{s, 3}}
$$

In fact, the sum of the squares of the two velocities yields the magnitude of the velocity vector after the incident shock and, thus, dividing by the speed of sound in region 3 yields the Mach number. When a reflected shock is observed, $M a_{r}$ is used to draw the shock polar corresponding to the reflected shock. The intersection of the shock polar of the reflected shock with the shock polar of the transmitted shock in the $(\theta, p)$-plane represents the final state and the solution of the oblique shock refraction problem. An alternative determination of $M a_{r}$ is provided by the relation

$$
M a_{r}^{2} \sin ^{2}\left(\beta_{i}-\theta_{i}\right)=\frac{2+\left(\gamma_{1}-1\right) M a_{i}^{2} \sin ^{2} \beta}{2 \gamma_{1}^{2} M a_{i}^{2} \sin ^{2} \beta_{i}-\left(\gamma_{1}-1\right)},
$$

where $\theta_{i}$ is the flow turning angle of the incident shock. Numerically, it is verified that both methods yield the same result.

When a reflected rarefaction wave is observed instead, the solution can still be represented in the $(\theta, p)$-plane. Across a rarefaction wave, the pressure decreases, the velocity increases, and the flow is turned by an angle $\theta_{r}$. This indicates that a functional relation exists between the pressure and the flow turning angle following a rarefaction wave. Such a relation enables the representation of the solution states along a curve in the $(\theta, p)$-plane corresponding to the rarefaction wave. The Prandtl-Meyer function provides a natural first candidate to establish this functional relation. The Prandtl-Meyer function $\Theta(M a)$ expresses the flow turning angle $\Theta$ as a function of the Mach number observed after the rarefaction wave; $\Theta(M a)$ measures the angle that a flow starting at $M a=1$ must turn via a rarefaction wave to reach the desired $M a$ speed. For a more complete discussion of the Prandtl-Meyer function, including its derivation, 
see Liepmann and Roshko [28]. Let $\lambda_{1}=\left(\gamma_{1}+1\right) /\left(\gamma_{1}-1\right)$, so that the Prandtl-Meyer function can be written as

$$
\Theta(M a)=\sqrt{\lambda_{1}} \tan ^{-1}\left(\sqrt{\frac{M a^{2}-1}{\lambda_{1}}}\right)-\tan ^{-1}\left(\sqrt{M a^{2}-1}\right) .
$$

The Prandtl-Meyer function now relates $M a_{3}, M a_{5}$, and the flow turning angle $\theta_{r}$ by

$$
\Theta\left(M a_{3}\right)=\Theta\left(M a_{5}\right)-\theta_{r} .
$$

Note that $\theta_{r}$ is subtracted from $\Theta\left(M_{5}\right)$ as a result of an expansion turn. In the above relation $M a_{3}$ is known, so that $\Theta\left(M_{3}\right)$ is known, $\theta_{r}$ can be considered the independent parameter of the curve, and $\mathrm{Ma}_{5}$ is the unknown. Therefore, the above relation can be solved to yield

$$
M a_{5}=\Theta^{-1}\left[\Theta\left(M a_{3}\right)+\theta_{r}\right]
$$

where $\Theta^{-1}$ represents the inverse function and $\theta_{r}$ satisfies the relation $\theta_{r}+\theta_{i}=\theta_{t}$. The isentropic relations yield the pressure $p_{5}$ as a function of $M a_{5}$ through the sequence

$$
\begin{aligned}
& \frac{T_{0}}{T_{5}}=1+\frac{\gamma_{1}-1}{2} M a_{5}^{2}, \\
& \frac{T_{0}}{T_{3}}=1+\frac{\gamma_{1}-1}{2} M a_{3}^{2}, \\
& \frac{p_{5}}{p_{0}}=\left(\frac{T_{0}}{T_{5}}\right)^{-\frac{\gamma_{1}}{\gamma_{1}-1}}, \\
& \frac{p_{0}}{p_{3}}=\left(\frac{T_{0}}{T_{3}}\right)^{\frac{\gamma_{1}}{\gamma_{1}-1}},
\end{aligned}
$$

and finally

$$
p_{5}=p_{3} \frac{p_{5}}{p_{0}} \frac{p_{0}}{p_{3}} .
$$

The above relation expresses $p_{5}=p_{5}\left(\theta_{r}\right)$. Therefore, the curve corresponding to the rarefaction wave in the $(\theta, p)$-plane can now be drawn.

Furthermore, from the isentropic relations it is possible to determine all quantities in region 5 ,

$$
\begin{aligned}
& \frac{\rho_{0}}{\rho_{5}}=\left(\frac{T_{0}}{T_{5}}\right)^{\frac{1}{\gamma_{1}-1}}, \\
& \frac{\rho_{0}}{\rho_{3}}=\left(\frac{T_{0}}{T_{3}}\right)^{\frac{1}{\gamma_{1}-1}}
\end{aligned}
$$

to obtain

$$
\begin{aligned}
T_{5} & =T_{3} \frac{T_{5}}{T_{0}} \frac{T_{0}}{T_{3}} \\
\rho_{5} & =\rho_{3} \frac{\rho_{5}}{\rho_{0}} \frac{\rho_{0}}{\rho_{3}} \\
c_{s, 5} & =\sqrt{\frac{\gamma_{1} p_{5}}{\rho_{5}}}
\end{aligned}
$$


The above procedure, although theoretically straightforward, can be implemented in practice only with difficulty. In particular, the principal obstacle lies in expressing $M a_{5}=M a_{5}\left(\theta_{r}\right)$ as it is very difficult to invert the Prandtl-Meyer function. A better approach is to treat $M a_{5}$ as the parameter and then determine $\theta_{r}\left(M_{5}\right)$ and $p_{5}\left(M_{5}\right)$. This approach is equivalent to the above approach, and will be further clarified in the examples to follow.

\section{The weighted essentially non-oscillatory (WENO) method}

The numerical simulations of regular shock refraction were performed using the HOPE (highorder polynomial expansion) code at the Lawrence Livermore National Laboratory [10]. The HOPE code is based on the high-order weighted essentially non-oscillatory (WENO) shockcapturing method [21, 4]. In the WENO method, a polynomial of order $r$ is used to reconstruct the spatially-discretized conservative fluxes of the Euler equations: as the polynomials may cross discontinuities and, thus, induce large Gibbs oscillations in the numerical solution, a weighted average of all the possible polynomial reconstructions at a point is computed. Smaller weights are assigned to polynomials crossing discontinuities and equal weights are assigned to polynomials over smooth regions. With this weighting, the formal order of accuracy for the derivative of the conservative flux is $2 r-1$. In the present study, the semi-discrete equations are evolved in time using the third-order total variation diminishing (TVD) Runge-Kutta method [37]. The implementation of the WENO method in the HOPE code is summarized in this section. Notable features of the HOPE code are also briefly summarized.

One of the main advantages of high-order shock-capturing WENO method is that it provides better resolution of complex flow features at long evolution times using fewer grid points than traditional higher-order methods. In particular, WENO methods of sufficiently highorder are among the least dissipative shock-capturing schemes, and are well-suited for the simulation of complex evolving flows containing structures with a wide range of scales. Furthermore, WENO the resolving power of the WENO method can be improved by optimizing the stencil and hybridizing with a central difference scheme to reduce the overall numerical dissipation [20]. WENO methods are also well-suited for non-constant grid spacing $\Delta x$ and for unstructured grids, and can be easily and systematically extended to arbitrarily high-order of accuracy.

\subsection{Overview of the WENO method implementation for the Euler equations}

An overview of the formally high-order WENO shock-capturing method in the HOPE code implementation is presented in this section. First, the two-dimensional compressible fluid dynamics equations for a single ideal gas are reviewed. The spatial discretization via highorder polynomials and flux-averaging are then discussed. Finally, the third-order Runge-Kutta time-evolution scheme for the spatially-discretized equations is briefly summarized.

\subsubsection{The two-dimensional Euler equations of gas dynamics}

In the absence of molecular dissipation and external forces, the equations solved in the present study are the two-dimensional compressible fluid dynamics equations for a single ideal gas. In two dimensions, the Euler equations form a hyperbolic system of four equations describing the conservation of mass, momentum, and energy. The unknowns are the density $\rho$, the momentum in the $x$ - and $y$-directions $\rho u$ and $\rho v$, and the (total) energy $E$. The equations can be written 


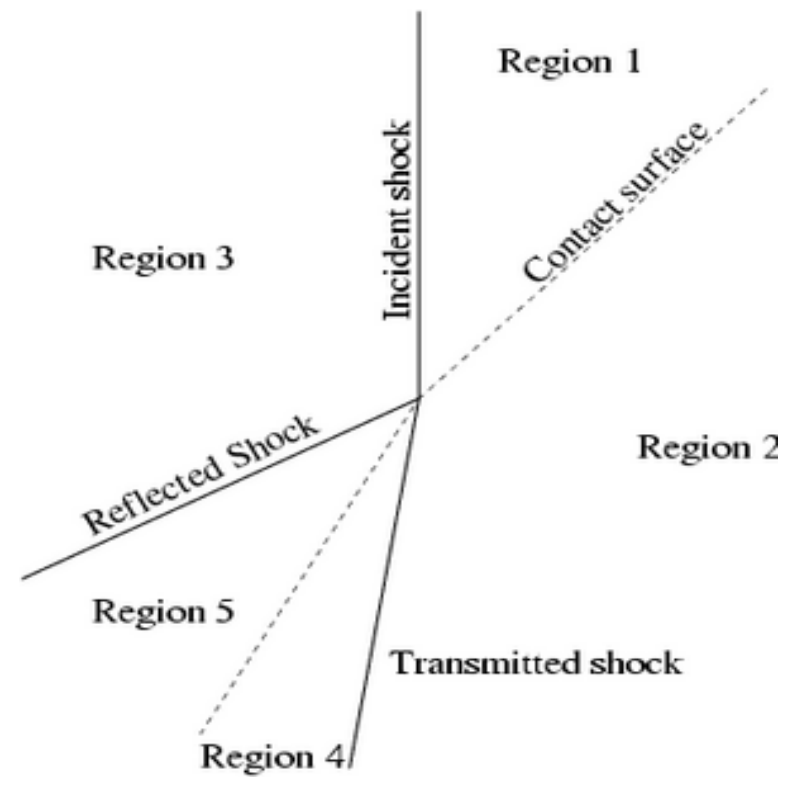

Figure 9: A shock wave interacting with a contact surface as observed in a reference frame moving with the point of contact. Note the reflected and the transmitted shocks.

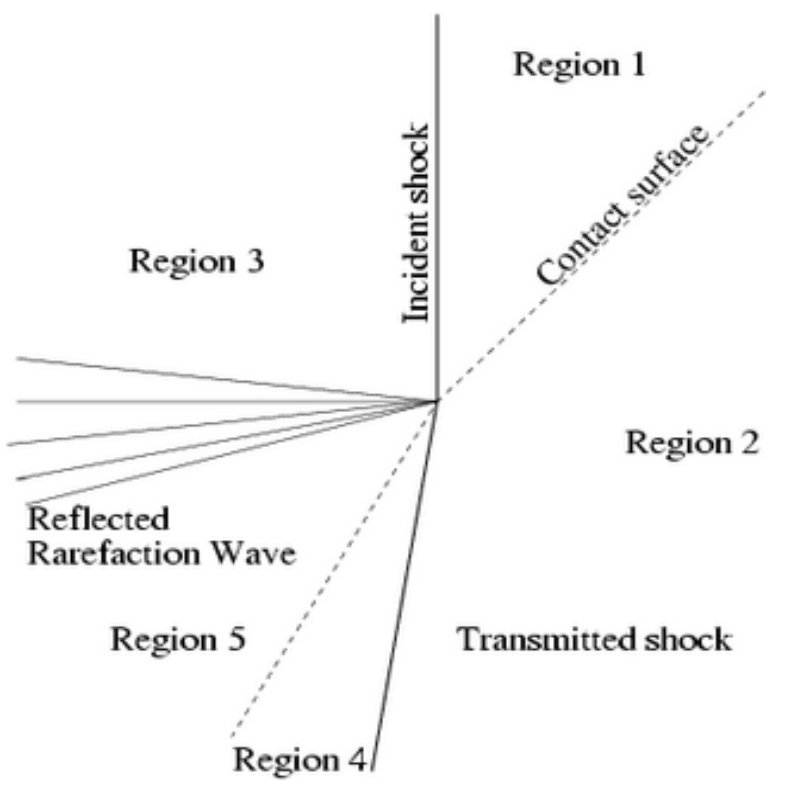

Figure 10: A shock wave interacting with a contact surface as observed in a reference frame moving with the point of contact. Note that the reflected wave is a rarefaction wave. 
as the system

$$
\frac{\partial}{\partial t}\left[\begin{array}{c}
\rho \\
\rho u \\
\rho v \\
E
\end{array}\right]+\frac{\partial}{\partial x}\left[\begin{array}{c}
\rho u \\
\rho u^{2}+p \\
\rho u v \\
(E+p) u
\end{array}\right]+\frac{\partial}{\partial y}\left[\begin{array}{c}
\rho v \\
\rho u v \\
\rho v^{2}+p \\
(E+p) v
\end{array}\right]=0
$$

As the additional unknown $p$ appears in the above formulation, a fifth equation relating the pressure to the energy and density $p=p(E, \rho)$ (equation of state) is needed to close the system. In the present study, it is always assumed that the fluids are ideal gases with equation of state given by the ideal gas law

$$
p=\rho R T,
$$

where $R$ is the ratio of the universal gas constant $R_{u}$ and the molecular weight of the gas $M$,

$$
R=\frac{R_{u}}{M}
$$

and $R_{u}=8.3143 \mathrm{~J} /(\mathrm{mol} \mathrm{K})$ or $R_{u}=8.3143 \times 10^{7} \mathrm{erg} /(\mathrm{mol} \mathrm{K})$ in cgs units. It is also assumed that the gas is polytropic, so that the internal energy is proportional to the temperature:

$$
U=c_{v} T
$$

where

$$
c_{v}=\frac{R}{\gamma-1}
$$

is the specific heat at constant volume.

The total energy is the sum of the kinetic energy and internal energy

$$
E=\frac{\rho\left(u^{2}+v^{2}\right)}{2}+\rho U .
$$

From the relations (43), (45), and (46), it follows that Eq. (47) can be written as

$$
E=\frac{\rho\left(u^{2}+v^{2}\right)}{2}+\frac{p}{\gamma-1},
$$

which yields the pressure

$$
p=(\gamma-1)\left[E-\frac{\rho\left(u^{2}+v^{2}\right)}{2}\right] .
$$

A gas satisfying the above relation is a gamma-law gas (see [27]).

\subsubsection{Predecessor of the flux-averaged WENO method: the ENO method and the cell-averaged WENO method}

Before describing the WENO method, it is useful to describe its predecessor-the essentially non-oscillatory (ENO) method. The ENO method belongs to a class of flux reconstruction methods introduced by Shu and Osher in 1988 [37, 38], although ENO reconstruction was first introduced by Harten et al. in 1987 [14]. These reconstruction procedures provide a new method for the spatial discretization of a system of hyperbolic conservation laws. A polynomial approximation to the primitive function of $u(x, t)$ is constructed, selecting the stencil that yields the least oscillation. 
In a typical semi-discrete formulation, the one-dimensional scalar conservation law

$$
\frac{\partial u}{\partial t}+\frac{\partial F(u)}{\partial x}=0
$$

is spatially discretized to obtain a system of first-order ordinary differential equations

$$
\frac{d u\left(x_{i}, t\right)}{d t}=-\frac{\widehat{F}_{i+1 / 2}-\widehat{F}_{i-1 / 2}}{\Delta x},
$$

where $\widehat{F}_{i+1 / 2}$ is the numerical flux approximating the continuum flux $F(u)$ [23].

The ENO method can be considered as an extension of an earlier method called the reconstruction via the primitive function introduced in the context of the piecewise-parabolic method (PPM) [7]. Reconstruction via the primitive function provides a method for constructing high-order accurate, point-wise approximations of $u(x, t)$ given the spatial averages $\bar{u}_{j}(t)$. Given a high-order approximation for $u(x, t)$, the high-order point-wise approximation can be used in the computation of the numerical flux. Define the primitive function

$$
\phi(x, t)=\int_{x_{1 / 2}}^{x} u(s, t) \mathrm{d} s,
$$

where $x_{1 / 2}$ is any arbitrary starting grid point. The key component of the method is that knowledge of the spatial averages of $u(x, t)$ gives exact point-wise values for $\phi(x, t)$ at the grid points. In particular, the relation

$$
\begin{aligned}
\phi\left(x_{i+1 / 2}, t\right) & =\int_{x_{1 / 2}}^{x_{i+1 / 2}} u(s, t) \mathrm{d} s \\
& =\Delta x \sum_{j=1}^{i} \bar{u}_{j}(t)
\end{aligned}
$$

holds. Therefore, a polynomial $P(x)$ approximating $\phi(x, t)$ to high-order accuracy can easily be reconstructed by choosing the grid points $\left(x_{i+1 / 2}, \phi\left(x_{i+1 / 2}, t\right)\right)$ as the interpolation points. For clarity, the time-dependence is subsequently omitted to illustrate the reconstruction at a given time. Choosing $r+1$ points in the stencil yields a polynomial $P(x)$ that approximates $\phi(x)$ to order $r+1$ :

$$
P(x)=\phi(x)+O\left[(\Delta x)^{r+1}\right],
$$

so that $p(x) \equiv d P(x) / d x$ is a polynomial approximating $u(x)$ such that

$$
p(x)=u(x)+O\left[(\Delta x)^{r}\right] .
$$

The ENO method [14] provides an algorithm for choosing the stencil that yields the least oscillation in a reconstruction via the primitive function method: this is achieved by choosing the point that yields the smallest divided-difference for a given choice of points for the polynomial reconstruction. Large values of the divided-differences indicate that a jump discontinuity is being crossed, which introduces large oscillations in the polynomial and considerably lowers the overall accuracy of the numerical solution. By avoiding such points, the ENO method achieves uniformly high-order accuracy in smooth flow regions and minimizes the oscillations that are inimical to high-order polynomial reconstruction methods. 
The above description of the ENO method is based on cell-averages $\bar{u}$. Shu and Osher [37] introduced a flux-averaged version of the ENO method such that a polynomial approximation to the primitive of the flux function $F(u)$ is reconstructed. In this method, it is assumed that $\bar{F}(u)$ is obtained as the spatial average of some function $F(u)$,

$$
\bar{F}_{i}=\frac{1}{\Delta x} \int_{x_{i-1 / 2}}^{x_{i+1 / 2}} F(s) \mathrm{d} s .
$$

Let $\phi(x)$ be the primitive of $F(u)$. As in Eq. (53), the sums of the cell averages constitute exact samples of the primitive function

$$
\begin{aligned}
\phi\left(x_{i+1 / 2}\right) & =\int_{x_{1 / 2}}^{x_{i+1 / 2}} F(s) \mathrm{d} s \\
& =\Delta x \sum_{j=1}^{i} \bar{F}_{j} .
\end{aligned}
$$

Now reconstruct a polynomial $P(x)$ that passes through $\left(x_{j}, \phi\left(x_{j}\right)\right)$. Choosing $r+1$ points yields an approximation of the form

$$
P(x)=\phi(x)+O\left[(\Delta x)^{r+1}\right],
$$

so that differentiating gives an $r$ th-order reconstruction

$$
p(x)=F(u)+O\left[(\Delta x)^{r}\right]
$$

for the flux function. Thus, just as for the cell-averaged ENO method, points that yield the overall smaller divided-difference are chosen using the flux averages instead.

Despite their success, ENO schemes have several drawbacks. For example, the stencil based on cell averages can be very sensitive to small round-off errors. This has the effect of yielding two different stencils for small errors. Furthermore, ENO schemes are based on complex logical statements that are not efficiently parallelizable. To overcome these drawbacks Liu, Osher and Chan developed the cell-averaged WENO method [29]. In this method, a convex combination of all possible stencils is formed, instead of choosing a single stencil among several possible stencils. Each stencil is assigned a weight that determines its relative contribution to the computed numerical flux. The weights are assigned so that stencils that cross discontinuities are given nearly zero weight, while stencils that are formed from points in smoother regions are given similar weights. Such schemes are easily parallelized. Another advantage of the WENO method is that the resulting flux is smoother than the flux obtained from the ENO method, and this property can be used to prove the convergence of the method for one-dimensional scalar conservation laws. The overall order of accuracy is also considerably improved as a result of the partial cancellation of truncation error terms.

The weighted average

$$
p(x)=\sum_{k=0}^{r-1} \frac{\widetilde{w}_{k}}{\sum_{l=1}^{r} \widetilde{w}_{l}} \frac{\mathrm{d} P_{k}(x)}{\mathrm{d} x},
$$

is used to determine the polynomial $p(x)$ through the point $x_{j}$ based on all of the possible $r-1$ reconstructions $P_{k}(x)$, where

$$
\widetilde{w}_{k}=\frac{\gamma_{k}}{\left(\epsilon+S_{j+k}\right)^{r}}
$$


are the weights, the $S_{j}$ are smoothness indicators, and $\epsilon=10^{-10}$ is a small number that regularizes the weights when $S_{j}=0$. The smoothness indicators are defined as

$$
S_{j}=\sum_{m=1}^{r-1} \frac{1}{m} \sum_{k=1}^{m}\left(\Delta^{r-m}\left[u_{j-r+k}\right]\right)^{2},
$$

where $\Delta^{i}\left[u_{j}\right]$ is the $i$ th divided-difference. The term $\widetilde{w}_{k} /\left(\sum_{l=1}^{r} \widetilde{w}_{l}\right)$ introduces the desired ENO property, as this term is $O(1)$ when the stencil crosses a smooth region but becomes extremely small when the stencil crosses a region containing a discontinuity. The constants (linear weights) $\left\{\gamma_{k}\right\}$ are chosen in order to achieve an improvement in the overall order of

accuracy of the method. The Liu, Osher and Chan formulation has overall $O\left[(\Delta x)^{r+1}\right]$ formal order of accuracy in smooth flow regions.

\subsubsection{The flux-averaged WENO method}

In 1996 Jiang and Shu [21] proposed significant improvements to the WENO method developed by Liu, Osher and Chan [29] including the use of a flux-averaged WENO formulation and a new method to measure the smoothness of the stencils. A flux-averaged WENO is preferred as it is simpler and less costly to implement for multi-dimensional formulations. Furthermore Jiang and Shu noticed that when WENO reconstruction is performed over a smooth region, all the $r$ stencils and $r-1$ points are considered and weighted equally. Therefore, an efficient use of the information should yield a method of formal order $2 r-1$, a significant improvement over the $r+1$ order of Liu, Osher and Chan.

Jiang and $\mathrm{Shu}$ focused on the smoothness indicator $S_{j}$ to improve the original WENO method. Liu, Osher and Chan defined $S_{j}$ in Eq. (62) in terms of divided-differences, whereas Jiang and Shu proposed a new definition based on the $L^{2}$ norm of the total variation

$$
S_{j}=\sum_{l=1}^{r-1} \int_{x_{j-1 / 2}}^{x_{j+1 / 2}}(\Delta x)^{2 l-1}\left[p_{j}^{(l)}\right]^{2} \mathrm{~d} x,
$$

where $p_{j}^{(l)}$ is the $l t h$-derivative of $p_{j}(x)$. The total variation is a better indicator of smoothness. This definition also has the advantage of taking into account the effect of high-order variations. Furthermore, this definition of smoothness produces the optimal WENO scheme. Therefore, for $r=3$ a fifth-order WENO approximation is obtained instead of the fourth-order approximation obtained by Liu, Osher and Chan. Note that in practice, all numerical methods degenerate to first-order accuracy at best in the vicinity of shocks and other discontinuities. While such loworder accuracy is inevitable in flows containing discontinuities, high-order methods still provide very good resolution of the discontinuities and other flow features, and are considerably less numerically dissipative than lower-order schemes. Thus, high-order methods can evolve a flow field in relatively smooth regions with less nonlinear, grid-dependent dissipation than a lowerorder method with the same time-evolution scheme and grid resolution. Consequently, highorder methods such as the WENO method are excellent candidates for large-eddy simulations of shock-induced mixing using explicit subgrid-scale modeling, particularly when hybridized with a non-upwinded, high-order scheme in smooth flow regions or modified using optimized stencils [20]. 


\subsubsection{The third-order TVD Runge-Kutta time-evolution scheme}

In addition to flux-averaged ENO, Shu and Osher [37] also introduced a class of high-order total-variation-diminishing (TVD) Runge-Kutta time-evolution schemes to advance a system of first-order differential equations in time. The total variation of a discrete scalar solution $u_{j}^{n}$, where $n$ is the timestep, is defined as the summation of extrema with maxima counted positively and minima counted negatively,

$$
T V\left(u^{n}\right)=\sum_{j}\left|u_{j+1}^{n}-u_{j}^{n}\right| .
$$

The total variation increases in the presence of additional oscillations or oscillations increasing in amplitude. The increase in oscillations cannot occur for conservation laws such as the Euler equations, and therefore a numerical scheme enforcing this condition is desirable. A numerical scheme is total-variation-diminishing (TVD) if the total variation at later timesteps is non-increasing,

$$
T V\left(u^{n+1}\right) \leq T V\left(u^{n}\right) .
$$

It is important to use a TVD scheme to ensure that large oscillations are not introduced at shocks and contact surfaces in the numerical solution. More importantly, the TVD property ensures stability of the numerical scheme, as first shown by Harten [13].

In many practical applications requiring a balance between temporal accuracy and computational efficiency, the third-order TVD Runge-Kutta scheme is found to be sufficient: this method is used in the present work. The third-order TVD Runge-Kutta scheme for the ordinary differential equation

$$
\frac{d u}{d t}=L(u)
$$

is given by

$$
\begin{aligned}
& u^{(1)}=u^{n}+\Delta t L\left(u^{n}\right) \\
& u^{(2)}=\frac{3}{4} u^{n}+\frac{1}{4} u^{(1)}+\frac{1}{4} \Delta t L\left(u^{(1)}\right) \\
& u^{n+1}=\frac{1}{3} u^{n}+\frac{2}{3} u^{(2)}+\frac{2}{3} \Delta t L\left(u^{(2)}\right)
\end{aligned},
$$

where $\Delta t$ is the timestep.

\subsubsection{Summary of algorithm}

The present implementation of the flux-averaged WENO method uses local Lax-Friedrichs flux-splitting and a characteristic decomposition of the variables and fluxes. An exact or approximate Riemann solver at each cell face is not used. A review of the characteristic projection method is given in [11]. At a given timestep, the numerical algorithm can be summarized schematically as follows:

1. compute the average state $\bar{\phi}^{\alpha}$ at $(i+1 / 2, j, k)$ using a Roe average (see $\S 5.3 .2$ in [23]);

2. evaluate the left and right eigenvector matrices $\mathbf{L}(\bar{\phi})$ and $\mathbf{R}(\bar{\phi})$, and the eigenvalues of the Jacobian matrix at the average state $\bar{\phi}^{\alpha}$;

3. for every stencil, project the conservative fields and the fluxes onto the local characteristic directions using the left eigenvector matrix $(2 r-1$ is the formal order of accuracy)

$$
\phi_{m, j, k}^{\alpha(c h)}=\mathbf{L}(\bar{\phi}) \phi_{m, j, k}^{\alpha}, \widehat{\mathbf{F}}_{m, j, k}^{\alpha(c h)}=\mathbf{L}(\bar{\phi}) \widehat{\mathbf{F}}_{m, j, k}^{\alpha}
$$

with $m \in[i-r+1, i+r]$; 
4. evaluate the left and right characteristic fluxes $\widehat{\mathbf{F}}_{m, j, k}^{\alpha(c h) \pm}$ point-wise using local or global Lax-Friedrichs flux-splitting;

5. reconstruct the numerical characteristic flux functions $\widehat{\mathbf{F}}_{m, j, k}^{\alpha(c h) \pm}$ from the point-wise values $\mathbf{F}_{m, j, k}^{\alpha}$ using the WENO method;

6. compute the numerical flux function in physical space by projecting back using the right eigenvector matrix

$$
\widehat{\mathbf{F}}_{i+1 / 2, j, k}^{\alpha}=\mathbf{R}(\bar{\phi})\left(\widehat{\mathbf{F}}_{i+1 / 2, j, k}^{\alpha,+}+\widehat{\mathbf{F}}_{i+1 / 2, j, k}^{\alpha,-}\right)
$$

7. obtain the fluxes in the $y$ - and $z$-directions $(i, j+1 / 2, k)$ and $(i, j, k+1 / 2)$, respectively, accordingly;

8. advance the solution one timestep using the third-order TVD Runge-Kutta scheme, and;

9. compute a new timestep based on the CFL criterion.

In terms of the characteristic projection of the numerical flux function, $\widehat{F}_{i+1 / 2}^{\alpha, n, \pm(c h)}$, the WENO reconstruction is applied by upwinding at the interface $i+1 / 2$ according to incoming or outgoing characteristics (or to the sign of the eigenvalues of the Jacobian at the interface):

$$
\begin{aligned}
& \widehat{F}_{i+1 / 2, j, k}^{\alpha, n(c h)}=\widehat{F}_{i+1 / 2, j, k}^{\alpha, n,+(c h)} \text { if } \lambda_{i+1 / 2, j, k}^{\alpha, n} \geq 0, \\
& \widehat{F}_{i+1 / 2, j, k}^{\alpha, n(c h)}=\widehat{F}_{i+1 / 2, j, k}^{\alpha, n,-(c h)} \text { if } \lambda_{i+1 / 2, j, k}^{\alpha, n}<0,
\end{aligned}
$$

where $\widehat{F}_{i+1 / 2, j, k}^{\alpha, n,+(c h)}$ and $\widehat{F}_{i+1 / 2, j, k}^{\alpha, n,-(c h)}$ are the fluxes reconstructed from $\widehat{F}_{i+1 / 2, j, k}^{\alpha, n(c h)}$ on stencils shifted in the left and right directions with respect to the interface $i+1 / 2$, respectively. For global Lax-Friedrichs flux-splitting,

$$
\begin{aligned}
\widehat{\mathbf{F}}_{i+1 / 2, j, k}^{\alpha, n(c h)}(\phi) & =\widehat{\mathbf{F}}_{i+1 / 2, j, k}^{\alpha, n(c h)+}(\phi)+\widehat{\mathbf{F}}_{i+1 / 2, j, k}^{\alpha, n(c h)-}(\phi), \\
\widehat{\mathbf{F}}_{i+1 / 2, j, k}^{\alpha, n(c h) \pm}(\phi) & =\frac{\widehat{\mathbf{F}}_{i+1 / 2, j, k}^{\alpha, n(c h)}(\phi) \pm \lambda \phi_{i+1 / 2, j, k}^{\alpha, n(c h)}}{2}
\end{aligned}
$$

with $\lambda \equiv \max \left(|\mathbf{v}|+c_{s}\right)$ the largest eigenvalue in absolute value of the Jacobian $\partial \mathbf{F}^{\alpha} / \partial \phi^{\beta}$ along the relevant direction. For local Lax-Friedrichs flux-splitting, the maximum is evaluated only along the neighborhood of a given grid point.

For example, in terms of the mth characteristic field, the fifth-order WENO method uses a linear combination of three, three-point sub-stencils

$$
\phi_{i+1 / 2, j, k, m}^{(c h) \pm}=\sum_{n=1}^{3} w_{n}^{ \pm} \phi_{i+1 / 2, j, k, m}^{(c h)(n) \pm},
$$

with

$$
\begin{aligned}
\phi_{i+1 / 2, j, k, m}^{(c h)(1) \pm} & =\frac{11 \phi_{i, j, k, m}^{(c h)}-7 \phi_{i \pm 1, j, k m}^{(c h)}+2 \phi_{i \pm 2, j, k, m}^{(c h)}}{6}, \\
\phi_{i+1 / 2, j, k, m}^{(c h)(2) \pm} & =\frac{2 \phi_{i \mp 1, j, k, m}^{(c h)}+5 \phi_{i, j, k, m}^{(c h)}-\phi_{i \pm 1, j, k, m}^{(c h)}}{6}, \\
\phi_{i+1 / 2, j, k, m}^{(c h)(3) \pm} & =\frac{-\phi_{i \mp 2, j, k, m}^{(c h)}+5 \phi_{i \mp 1, j, k, m}^{(c h)}+2 \phi_{i, j, k, m}^{(c h)}}{6},
\end{aligned}
$$


the nonlinear weights

$$
w_{m}^{ \pm}=\frac{\widetilde{w}_{m}^{ \pm}}{\sum_{n=1}^{3} \widetilde{w}_{n}^{ \pm}},
$$

where

$$
\widetilde{w}_{1}^{ \pm}=\frac{\gamma_{1}}{\left(\epsilon+S_{1}^{ \pm}\right)^{2}}, \quad \widetilde{w}_{2}^{ \pm}=\frac{\gamma_{2}}{\left(\epsilon+S_{2}^{ \pm}\right)^{2}}, \quad \widetilde{w}_{3}^{ \pm}=\frac{\gamma_{3}}{\left(\epsilon+S_{3}^{ \pm}\right)^{2}}
$$

with the linear weights

$$
\gamma_{1}=\frac{1}{10}, \gamma_{2}=\frac{3}{5}, \gamma_{3}=\frac{3}{10}
$$

and the smoothness indicators

$$
\begin{aligned}
S_{1}^{ \pm} & =\frac{1}{4}\left[3 \phi_{i, j, k, m}^{(c h)}-4 \phi_{i \pm 1, j, k, m}^{(c h)}+\phi_{i \pm 2, j, k, m}^{(c h)}\right]^{2}+\frac{13}{12}\left[\phi_{i, j, k, m}^{(c h)}-2 \phi_{i \pm 1, j, k, m}^{(c h)}+\phi_{i \pm 2, j, k, m}^{(c h)}\right]^{2},(81 \\
S_{2}^{ \pm} & =\frac{1}{4}\left[\phi_{i \pm 1, j, k, m}^{(c h)}-\phi_{i \mp 1, j, k, m}^{(c h)}\right]^{2}+\frac{13}{12}\left[\phi_{i \mp 1, j, k, m}^{(c h)}-2 \phi_{i, j, k, m}^{(c h)}+\phi_{i \pm 1, j, k, m}^{(c h)}\right]^{2}, \\
S_{3}^{ \pm} & =\frac{1}{4}\left[\phi_{i \mp 2, j, k, m}^{(c h)}-4 \phi_{i \mp 1, j, k, m}^{(c h)}+3 \phi_{i, j, k, m}^{(c h)}\right]^{2}+\frac{13}{12}\left[\phi_{i \mp 2, j, k, m}^{(c h)}-2 \phi_{i \mp 1, j, k, m}^{(c h)}+\phi_{i, j, k, m}^{(c h)}\right]^{2}
\end{aligned}
$$

(see $[21,36]$ ). See [4] for the corresponding formulae for the seventh- and higher-order WENO schemes. Other flux-split methods such as Marquina flux-splitting [31], can also be used. Given a choice of flux-splitting, weights, and smoothness indicators (as shown above), there are no tunable algorithmic parameters in the WENO method.

\subsection{The (High-Order Polynomial Expansion) HOPE code}

Although the HOPE code was presently used to investigate two-dimensional shock refraction, the code is a framework for the multi-dimensional numerical simulation of the fully-nonlinear evolution of hydrodynamic instabilities and late-time mixing generated by single- and multimode Richtmyer-Meshkov, Rayleigh-Taylor, and Kelvin-Helmholtz instabilities [10]. The nonlinear system of hyperbolic partial differential equations is solved in one, two, or three spatial dimensions by the high-order characteristics-based WENO method. HOPE is a single-fluid (single-gamma implementation) and a two-fluid (gamma-blended) code. Both of these implementations are used and validated in the present investigation.

\subsubsection{Overview of the HOPE code}

Using the original multi-dimensional parallel WENO method for the Euler equations as a template, additional functionality has been added in the HOPE code. The HOPE code is written in Fortran 90/95 and is fully parallelized using the Message Passing Interface (MPI) and OpenMP with parallelization directives. The code is highly modular: the majority of the subroutines are built as modules and compiled into library form as part of the software library PseudoPack developed by Wai-Sun Don and Bruno Costa over the past 15 years. The library performs many basic optimized functions/operations for high-order WENO methods and Spectral methods on various serial machines such as Windows-Intel PCs with Fortran 90/95 compilers and parallel machines that support the MPI and/or OpenMP communication protocol. A makefile is used to compile the PseudoPack library and the HOPE code on a wide array of platforms. Users can compile the library once on a given computational platform and subsequently link with the required subroutines of interest from their own program. The HOPE code automatically maps 
the physical processors according to user preference if expressed as a three-dimensional Cartesian logical processor grid, thus minimizing the communication cost. Nearly linear speedup was achieved for large-scale simulations. With the support of the PseudoPack_2004 library, users can call any of the available subroutines in the library in lieu of subroutines coded in the HOPE code. For example, the third-order TVD Runge-Kutta time-evolution scheme used now in the HOPE code can be easily replaced with a fourth-order, five-stage Runge-Kutta scheme with the same arguments, thus increasing the overall temporal accuracy. The input files are modularized and organized by case study, so that only relevant files for the specific case under investigation need to be modified.

Three output data formats are currently supported: Tecplot ${ }^{1}$, PLOT3D, and Matlab ${ }^{2}$. The Tecplot data format allows rapid visual rendering of large multiple data sets and fine control of graphics output. The Matlab data format allows the analysis and easy manipulation of the simulation data using the mathematical functionalities of Matlab. With its wide spread usage and support, the PLOT3D data format allows importation of the data into other visualization software such as EnSight ${ }^{3}$. Furthermore, data may be saved in one large file or, if desired, in smaller distributed files according to the processor number. The restart module allows the user to manually restart using saved data from either a chosen saved step number or automatically from the last saved data dump. This allows users to write a script to automate and schedule a long-time simulation with minimum user intervention.

\subsubsection{Features of the HOPE code}

The HOPE code has the following useful features and flexibility.

\section{High-order WENO reconstruction}

The order of the WENO polynomial reconstruction algorithm can be specified. Currently third-, fifth-, seventh-, ninth-, and eleventh-order are all available. Both local and global Lax-Friedrich flux-splitting is implemented.

\section{Adaptive domain algorithm}

For certain classes of problems in which the solutions remain constant in large regions of the physical domain until late times, an adaptive domain algorithm is implemented in the HOPE code that automatically enlarges the size of the domain when changes from the constant state are detected. This user selectable capability allows a simulation to start with a small number of grid points in a smaller initial physical domain during the early time period instead of computing the solution in far-field regions that remain unchanged until much later in time. For example, in a long-time integration of the RichtmyerMeshkov instability in a long shock tube, the region of interest at early times is confined to a rather small region at one end of the physical domain, so that one can begin the calculation with a sufficiently small physical domain and extend the domain continuously when the algorithm detects changes in a designated variable such as density when the shock reaches the downstream boundary of the domain until the end of the physical domain has been reached. The coupling of the hybrid WENO/central-difference scheme and the adaptive domain algorithm enhancement provide a significant improvement in

\footnotetext{
${ }^{1}$ Tecplot is a trademark of AmTec Engineering

${ }^{2}$ Matlab is a trademark of MathWorks

${ }^{3}$ EnSight is a trademark of Computational Engineering International
} 
the computational efficiency of the HOPE code, especially for large-scale and long-time simulations.

\section{Interfacial specifications}

Different initial interfacial perturbations can be specified in addition to a single-mode perturbation. The following two- and three-dimensional extensions (unless specified otherwise) are included. For each grid point $(x, y, z)$ define an interface $\eta(y, z)$ (the shock is assumed to propagate in the $x$-direction):

- multi-mode perturbation

$$
\eta(y, z)=\sum_{m, n=1}^{N} a_{m n} \cos \left[\frac{n_{y}(m, n) \pi y}{L_{y}}+\theta_{y}(m, n)\right] \cos \left[\frac{n_{z}(m, n) \pi z}{L_{z}}+\theta_{z}(m, n)\right],
$$

where, for each index $m$ and $n, a_{m n}$ are the amplitudes, $n_{y}(m, n)$ and $n_{z}(m, n)$ are the integer mode numbers, $L_{y}$ and $L_{z}$ are the domain lengths in the $y$ and $z$ direction, and $\theta_{y}(m, n)$ and $\theta_{z}(m, n)$ are the phase angles;

- inclined interface

$$
\eta(y, z)=\frac{y-y_{0}}{\tan \phi_{y}}+\frac{z-z_{0}}{\tan \phi_{z}}
$$

where $y_{0}=L_{y} / 4, z_{0}=L_{z} / 4$, and $\phi_{y}$ and $\phi_{z}$ are the angles with respect to the horizontal $x$-axis;

- three-dimensional membrane model

$$
\eta(y, z)=a\left[\left|\sin \left(\frac{n_{y} \pi y}{L_{y}}\right) \sin \left(\frac{n_{z} \pi z}{L_{z}}\right)\right|-\cos \left(\frac{n_{y} \pi y}{L_{y}}\right) \cos \left(\frac{n_{z} \pi z}{L_{z}}\right)\right],
$$

where $a$ is the amplitude.

- three-dimensional random perturbation: given the perturbation amplitude $a$, wavenumber band, and root mean square, a random perturbation is defined that satisfies a specified power-law energy spectrum, and;

- multi-elliptical bubbles: define a Heaviside-like function $S$ and for each bubble index $n, R_{n}(x, y, z)$ and $S(x, y, z)$ will delineate the interior and the exterior of the ellipse with $S=1$ and $S=0$, respectively, at each grid point. The interface can further be modified by diffusing the interface over several grid points by an exponentiallydecaying function

$$
S(x, y, z)= \begin{cases}1 & R \leq 0 \\ \exp \left(-\alpha|R|^{\beta}\right) & 0<R<1 \\ 0 & R \geq 1\end{cases}
$$

where $\alpha=-\ln \epsilon, \epsilon$ is machine zero, $\beta$ is the order of the decay (typically, $\beta=8$ ), and $R$ is some measure of the distance of a given location to the interface: for example,

$$
R=\frac{S(x, y, z)+\delta-x}{2 \delta}
$$


where $\delta$ is the user specified diffuse interface thickness. It should be noted that the interface functions defined above are additive in the sense that one can select any combination of the interface specifications together to form the desired interface. The only exception is the multi-elliptical bubble case.

\section{Arbitrary gas specifications}

Users can specify the density, molecular weight for both gases. In addition, in the singlefluid implementation a single value of the adiabatic exponent $\gamma$ is further specified. For the two-fluids (gamma-blending) implementation both values of $\gamma$ are specified.

\section{Addition of mass fraction equation}

The Euler equations are augmented by an additional equation for the conservation of mass fraction for the first gas. In two dimensions, the system of Euler equations becomes

$$
\frac{\partial}{\partial t}\left[\begin{array}{c}
\rho \\
\rho u \\
\rho v \\
E \\
\rho m
\end{array}\right]+\frac{\partial}{\partial x}\left[\begin{array}{c}
\rho u \\
\rho u^{2}+p \\
\rho u v \\
(E+p) u \\
\rho m u
\end{array}\right]+\frac{\partial}{\partial y}\left[\begin{array}{c}
\rho v \\
\rho u v \\
\rho v^{2}+p \\
(E+p) v \\
\rho m v
\end{array}\right]=0,
$$

where the mass fraction $m$ is used to track the movement of the interface between two fluids in time and in mixing analysis [24].

\section{Gamma-blending for multiple fluids}

'Gamma-blending' [22,3] is used to simulate the flow of two different ideal gases with different adiabatic exponents. In this formulation the mass fraction $m$ is used to model the thermodynamic properties in the mixing region. The spatially-varying adiabatic exponent $\gamma(x, y)$ is given by

$$
\gamma=\frac{m c_{p_{1}}+(1-m) c_{p_{2}}}{m c_{v_{1}}+(1-m) c_{v_{2}}}
$$

\section{Analysis package}

An Analysis package for computing the statistics and spectra of the fields obtained from the simulations and to evaluate models for turbulent transport and mixing is available. The package is structured so as to be easily extendible to compute other quantities of interest by using the templates provided.

\section{Hybrid WENO-central difference scheme}

To further enhance the efficiency and reduce the intrinsic numerical dissipation of the WENO method, the HOPE code is capable of employing the hybrid WENO/centraldifference scheme that selectively switches locally at each grid point between the WENO and central-difference scheme using multi-resolution analysis. The central-difference scheme is of the same order as the WENO scheme used. It has been demonstrated that the hybrid scheme can significantly reduce the computational time in some simulations. Additional numerical simulations should be performed to evaluate the accuracy and other properties of this hybrid scheme. 


\section{Change of reference frame}

A built-in option allows the user to change reference frame, with the speed of the reference frame specified via the optional input parameters. This feature is useful if one wishes to follow some specific features of the flow field, such as a shock or an evolving interface.

\section{Available boundary conditions}

The built-in boundary conditions in any direction are periodic, reflecting, supersonic inflow, and supersonic outflow boundaries. The reflecting boundary condition simplify reverses the normal to the boundary component of the velocity field, and is particularly useful in simulating Richtmyer-Meshkov instabilities involving reshock from the end of a shock tube wall.

\section{Numerical simulations of shock refraction}

In this section, data from numerical simulations of regular shock refraction performed with the HOPE code is compared with the analytical predictions from $\S 2.2 .1$ and $\S 2.2 .2$. Two implementations of the HOPE code are used in the investigation. First, the case of argon and xenon gases (having the same adiabatic exponent $\gamma$ ) is investigated with the single-fluid (single-gamma) HOPE implementation. Presented are results for a shock in argon refracting into xenon ('light-to-heavy') and for a shock in xenon refracting into argon ('heavy-to-light'). Configurations for normal and oblique shock incidence are also considered. Second, the case of air(acetone) and sulfur hexafluoride $\left(\mathrm{SF}_{6}\right)$ gases (with different adiabatic exponents) is investigated using the two-fluid (gamma-blending) HOPE implementation. Presented are results for a shock in air(acetone) refracting into $\mathrm{SF}_{6}$ ('light-to-heavy') and for a shock in $\mathrm{SF}_{6}$ refracting into air(acetone) ('heavy-to-light'). Configurations for normal and oblique shock incidence are considered.

For all configurations considered, the gases are initially at rest, $u_{1}=u_{2}=0$. Specified in Table 1 are the initial densities $\rho_{1}$ and $\rho_{2}$, the adiabatic exponents $\gamma_{1}$ and $\gamma_{2}$, and the molecular weights. Furthermore the temperature in region 1 is fixed at $T_{1}=296 \mathrm{~K}$. The shock is always specified to have speed $M a_{1}=1.2$ in region 1 . For all configurations, the results are presented as follows. First the values of density, pressure, speed of sound, temperature, and flow velocity are obtained from the shock polar method described in $\S 2.2 .1$ and $\S 2.2 .2$. Plots of the shock polar, either in the $(u, p)$-plane for normal shock refraction or in the $(\theta, p)$-plane for oblique shock refraction, are displayed. Images from the simulations illustrating the wave structure are presented. Finally, a table with the analytical and numerical results and the percentage error is presented. In all of the cases considered, the numerical results are in excellent agreement with the theoretical predictions.

\subsection{Initial conditions}

The initial conditions require the specification of the physical properties of the gases, the shock Mach number, and the the initial perturbation. Additionally, the grid resolution, the length of the domain, the order of reconstruction, and the CFL number must also be specified. For the simulations presented here, the shock Mach number is $M a=1.2$. The initial temperature in region 1 is specified as $T_{1}=296 \mathrm{~K}$. The pressure and temperature in region 2 are obtained from the equation of state. The physical properties of the gas to be specified are the densities, 
the molecular weights, and the adiabatic exponents $\gamma_{i}$. All other quantities are derived from the ideal gas equation of state.

Two pairs of gases are considered in this investigation,

1. argon and xenon with $\gamma_{1}=\gamma_{2}=5 / 3$ and;

2. a mixture of $75 \%$ air and $25 \%$ acetone by volume denoted air(acetone) and sulfur hexafluoride $\left(\mathrm{SF}_{6}\right)$ with $\gamma_{1}=1.276$ and $\gamma_{2}=1.093$, respectively.

These choices of gases allow the validation of the implementations of single-gamma and gammablending in the HOPE code. The choice of shock Mach number and of gases [air(acetone) and $\mathrm{SF}_{6}$ ] is motivated by concurrent numerical simulations of the single-mode Richtmyer-Meshkov instability shock tube experiments of Collins and Jacobs [8] (see Part 2 [24]).

The properties of argon, xenon, air, and $\mathrm{SF}_{6}$ are obtained from the NIST Chemistry WebBook (see http://webbook.nist.gov/chemistry/). The properties of the air(acetone) mixture are computed using mass fractions for mixtures. The density of the air(acetone) mixture is

$$
\rho_{a a}=0.75 \rho_{a i r}+0.25 \rho_{a c},
$$

where $a c$ denotes acetone. All other quantities are computed using the acetone mass fraction

$$
m=\frac{0.25 \rho_{a c}}{\rho_{a a}} .
$$

The mass fraction of air in the mixture is $1-m$.

The heat capacity at constant pressure and at constant volume, the adiabatic exponent, the gas constant, and the molecular weight can then be determined from the ideal gas relations

$$
\begin{aligned}
c_{p, a a} & =m c_{p, a c}+(1-m) c_{p, a i r} \\
c_{v, a a} & =m c_{v, a c}+(1-m) c_{p, a i r}, \\
\gamma_{a a} & =\frac{c_{p, a a}}{c_{v, a a}} \\
R_{a a} & =c_{p, a a}-c_{v, a a} \\
M_{a a} & =\frac{R_{u}}{R_{a a}}
\end{aligned}
$$

where $R_{u}$ is the universal gas constant. The results from applying these relations to determine the properties of the mixture are presented in Table 1.

The flow ahead of the shock is initially at rest with $u_{1}=u_{2}=0$. The computational domain has size $10 \mathrm{~cm} \times 15 \mathrm{~cm}$, with an initial spatial resolution of $N_{x} \times N_{y}=500 \times 750$ grid points. This resolution corresponds to a uniform grid spacing in the $x$ - and $y$-directions of $\Delta x=\Delta y=0.02 \mathrm{~cm}$. The simulations were run to time $t=0.15 \mathrm{~ms}$. Computations were conducted on IBM Blue Pacific with 16 processors. The run time for the simulations was approximately two minutes. The CFL number was 0.45 . The shock was initially located at a distance of $3 \mathrm{~cm}$ from the end of the domain, and the interface was located at a distance of 5 $\mathrm{cm}$. A comparison of simulations with different grid resolutions showed no significant effects on the results. 


\begin{tabular}{|c|c|c|c|c|c|c|}
\hline & $\begin{array}{c}M \\
(\mathrm{~g} / \mathrm{mol})\end{array}$ & $\begin{array}{c}\rho \\
\left(\mathrm{g} / \mathrm{cm}^{3}\right)\end{array}$ & $\gamma$ & $\begin{array}{c}R \\
\mathrm{erg} /(\mathrm{g} \mathrm{K})\end{array}$ & $\begin{array}{c}c_{p} \\
\mathrm{erg} /(\mathrm{g} \mathrm{K})\end{array}$ & $\begin{array}{c}c_{v} \\
\mathrm{erg} /(\mathrm{g} \mathrm{K})\end{array}$ \\
\hline \hline argon & 39.948 & $1.67 \times 10^{-3}$ & 1.6667 & $2.0813 \times 10^{6}$ & $5.2033 \times 10^{6}$ & $3.122 \times 10^{6}$ \\
xenon & 131.3 & $5.584 \times 10^{-3}$ & 1.6667 & $6.3324 \times 10^{5}$ & $1.5831 \times 10^{6}$ & $9.4986 \times 10^{5}$ \\
air & 28.95 & $1.202 \times 10^{-3}$ & 1.4 & $2.872 \times 10^{6}$ & $1.0052 \times 10^{7}$ & $7.18 \times 10^{8}$ \\
acetone & 58.08 & $2.404 \times 10^{-3}$ & 1.1246 & $1.4315 \times 10^{6}$ & $1.2917 \times 10^{7}$ & $1.1485 \times 10^{7}$ \\
air(acetone) & 35.925 & $1.351 \times 10^{-3}$ & 1.276 & $2.3144 \times 10^{6}$ & $1.07 \times 10^{7}$ & $8.3854 \times 10^{6}$ \\
$\mathrm{SF}_{6}$ & 146.05 & $5.494 \times 10^{-3}$ & 1.093 & $5.6929 \times 10^{5}$ & $6.6906 \times 10^{6}$ & $6.1213 \times 10^{6}$ \\
\hline
\end{tabular}

Table 1: Properties of gases used in the HOPE shock refraction simulations. Values for the density, adiabatic exponent $\gamma$ and molecular weight are required.

\subsection{Boundary Conditions}

The boundary conditions were set to inflow and outflow for the left and right $x$-direction, respectively. Periodic boundary conditions were specified for the $y$-direction in the normal shock refraction investigation. For oblique shock refraction, the boundary conditions in the $y$ direction were specified to simulate an infinitely long oblique interface: the bottom $y$ boundary had rigid wall boundary conditions while the top wall had outflow boundary conditions with an additional quantity tracking the location of the shock.

\subsection{Refraction for gases with the same adiabatic exponent: the case of argon and xenon}

Simulations with argon and xenon having adiabatic exponents $\gamma=5 / 3$ are performed to validate the single-gamma implementation of the HOPE code.

\subsubsection{Normal shock refraction at an Ar-Xe interface with a reflected shock}

From the specified initial conditions, the flow conditions in regions 1 and 2 can be obtained as follows. First determine $R_{A r}=R_{u} / M_{A r}$ and $R_{X e}=R_{u} / M_{X e}$, where $R_{u}$ is the universal gas constant. From $R_{A r}$ and the initial temperature $T_{1}$ compute the sound speed $c_{s, 1}=\sqrt{\gamma_{1} R_{A r} T_{1}}$ and the pressure $p_{1}=\rho_{1} R_{A r} T_{1}$. It is assumed that $p_{2}=p_{1}$. Therefore, the temperature in region 2 is obtained from the equation of state with $T_{2}=p_{2} /\left(\rho_{2} R_{X e}\right)$. Note that the gases in region 1 and 2 are at different temperatures. Such a state is very difficult to configure experimentally. If both gases were at the same temperature a slight mismatch in the pressure would be observed. Following the matching conditions for contact surfaces in the numerical simulations, the pressure is initially matched; therefore, a small mismatch in the temperature is assumed.

The quantities in region 3 can be computed from the shock jump conditions. Next, from the matching conditions at the refracted contact surface, $u_{4}=u_{5}$ and $p_{4}=p_{5}$. Therefore, the velocities

$$
\begin{aligned}
& u_{5}=u_{3}-\phi_{r}\left(p_{5}\right) \\
& u_{4}=\phi_{t}\left(p_{4}\right)
\end{aligned}
$$

are matched to obtain

$$
u_{3}-\phi_{r}\left(p_{5}\right)=\phi_{t}\left(p_{5}\right)
$$


The above equation can be solved for $p_{5}$, and in turn for $u_{5}$. This method is graphically illustrated with shock polars in Fig 11. Next, the shock Mach numbers for the reflected and the transmitted shocks are determined via the pressure jump relation (9):

$$
\begin{aligned}
M a_{r} & =\sqrt{\frac{p_{5}\left(\gamma_{1}+1\right)}{2 p_{3} \gamma_{1}}+1} \\
M a_{t} & =\sqrt{\frac{p_{4}\left(\gamma_{2}+1\right)}{2 p_{2} \gamma_{2}}+1} .
\end{aligned}
$$

All other quantities in regions 4 and 5 follow from the normal shock relations.

Note that by construction, the pressures and the velocities in regions 1 and 2 and in regions 4 and 5 are matched. Note that the initial mismatch in temperature is very small with $\Delta T=T_{1}-T_{2}=2.5 \mathrm{~K}$. Also note that the reflected shock with Mach number $M a_{r}=1.04946$ is very weak.

Images from the WENO simulation illustrating the Ar-Xe normal shock refraction are presented in Fig. 12. Presented are contour plots for the density, pressure and velocity at the initial time $t=0 \mathrm{~ms}$ showing the incident shock and at time $t=0.15 \mathrm{~ms}$ showing the reflected and transmitted shocks. The plots at $t=0$ show the incident shock entering a region where both gases are at rest and have the same pressure. Behind the shock, the pressure increases and the gas is set in motion. The plots at $t=0.15$ show that as the reflected shock returns back into argon, the shock decelerates the flow set in motion by the initial incident shock. The reflected shock further increases the pressure, and the transmitted shock sets the xenon in motion. Following refraction, both the argon and xenon move with the same horizontal velocity.

From the WENO simulations the density, pressure, and flow velocities in all five regions are determined. The temperatures and speed of sound are computed using these quantities. The numerical results are presented together with the analytical results in Table 3 . The analytically computed Mach numbers for the system of shocks are summarized in Table 2. The analytical and numerical results are in excellent agreement.

\subsubsection{Normal shock refraction at an Xe-Ar interface with a reflected rarefaction wave}

Now consider the case with a $M a=1.2$ shock in xenon refracting at a Xe-Ar interface. The transition is now from the heavier gas (xenon) to the lighter gas (argon), generating a reflected rarefaction wave. As above, the initial data consists of the densities of the two gases, the molecular weights, and the temperature of xenon $T_{1}=296 \mathrm{~K}$. All quantities in regions 1 and 2 are determined from this information. Note that the temperature in region 2 obtained from the equation of state is now higher. All quantities in region 3 can be determined from the normal shock relations. From matching conditions for a contact surface, the velocities

$$
\begin{aligned}
& u_{4}=\phi_{t}\left(p_{4}\right) \\
& u_{5}=u_{3}-\psi_{r}\left(p_{5}\right)
\end{aligned}
$$

are matched to obtain

$$
\phi_{t}\left(p_{5}\right)=u_{3}-\psi_{r}\left(p_{5}\right) .
$$




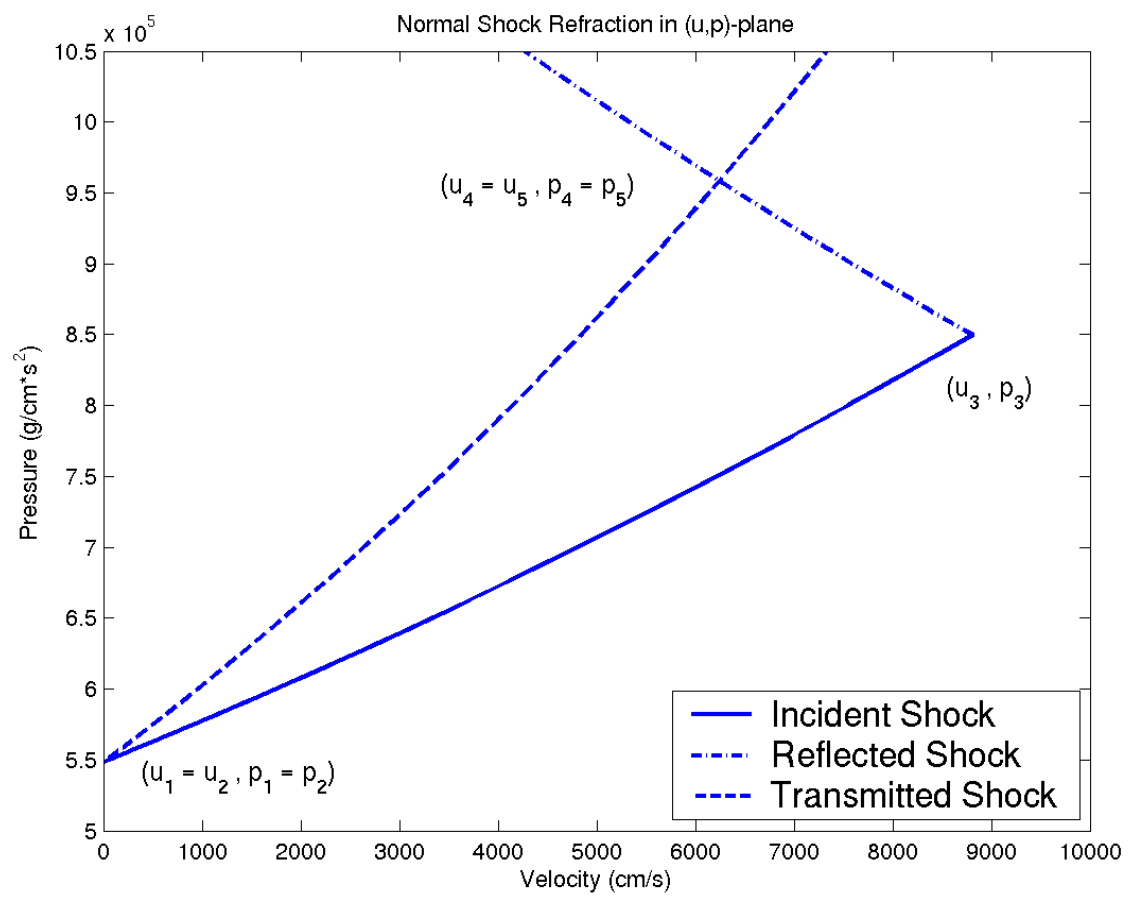

Figure 11: Normal shock refraction of a $M a=1.2$ shock wave in Ar interacting with a contact surface separating Ar and Xe. The refraction process is shown in the $(u, p)$-plane. The intersection of the curve representing the reflected shock with the curve representing the transmitted shock constitutes the solution of the refraction process.

\begin{tabular}{|c|c|c|}
\hline & $M a$ & $u_{\text {shock }}=c_{s} M a(\mathrm{~cm} / \mathrm{s})$ \\
\hline \hline Incident shock & 1.2 & 38,451 \\
Reflected shock & 1.04946 & 36,757 \\
Transmitted shock & 1.26374 & 22,242 \\
\hline
\end{tabular}

Table 2: Analytical results for the shock Mach numbers and the shock velocities in the normal Ar-Xe refraction. Note that the speed of the shock is reduced after refracting at the material interface. Therefore, this is a fast-slow refraction. 


$$
t=0 \mathrm{~ms}
$$
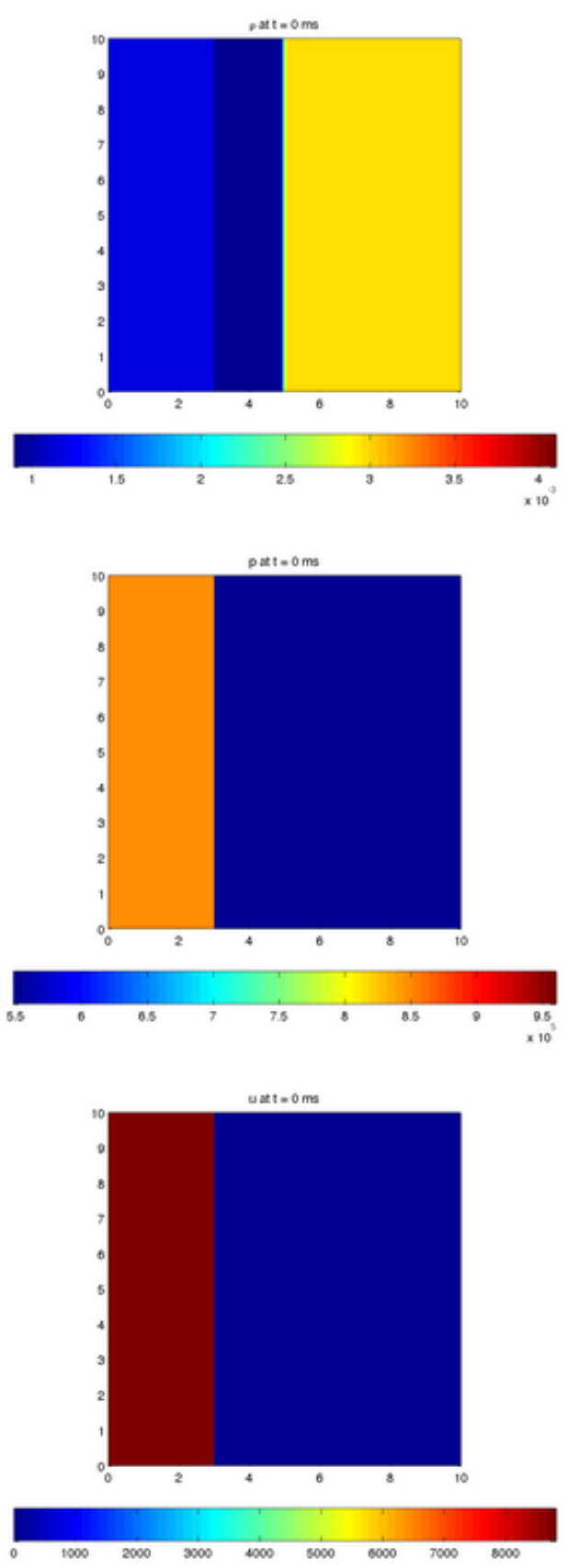

$$
t=0.15 \mathrm{~ms}
$$
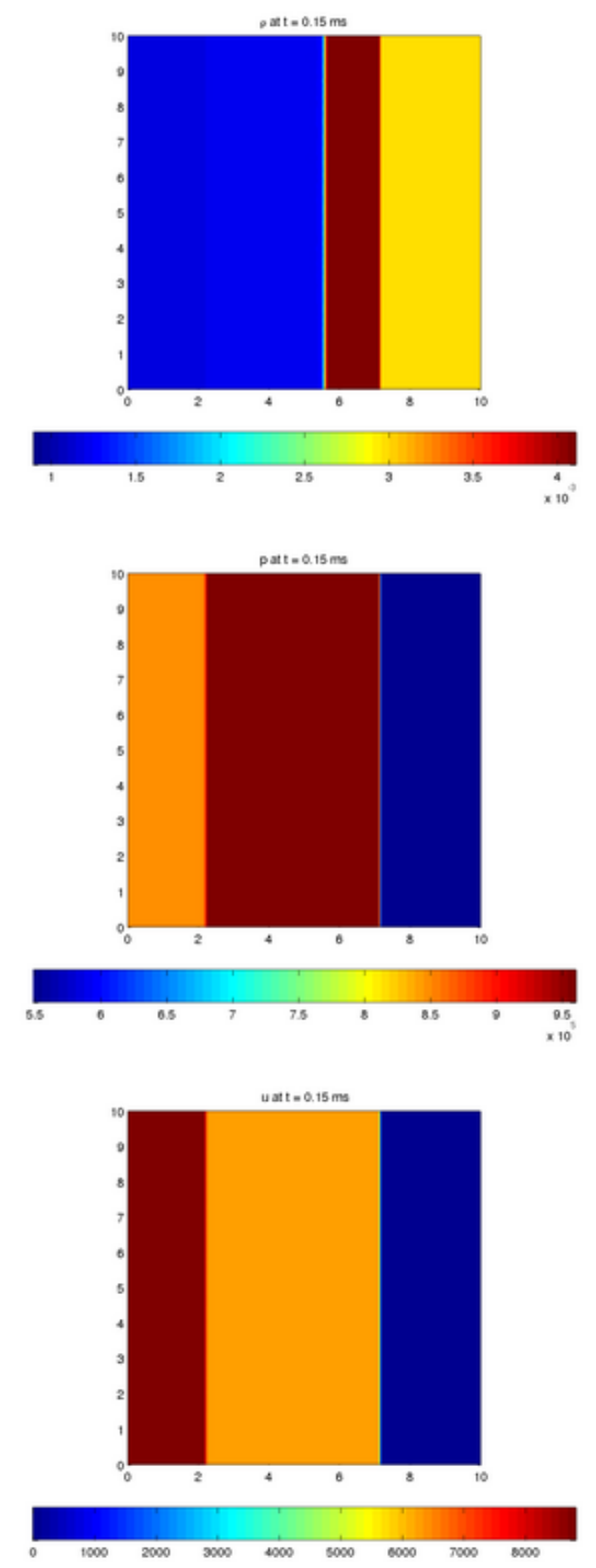

Figure 12: The density, pressure, and velocity $u$ at times $t=0$ and $0.15 \mathrm{~ms}$ from a WENO simulation of the Ar-Xe shock refraction for a $M a=1.2$ shock. The incident shock is shown at $t=0 \mathrm{~ms}$, and the reflected and transmitted shock waves are shown at $t=0.15 \mathrm{~ms}$. 


\begin{tabular}{|c|c|c|c|}
\hline \multicolumn{4}{|c|}{ Region 1 } \\
\hline \hline & Analytical values & WENO results & Error $(\%)$ \\
\hline$\rho\left(\mathrm{g} / \mathrm{cm}^{3}\right)$ & $8.9 \times 10^{-4}$ & $8.9 \times 10^{-4}$ & 0 \\
$p\left(\mathrm{~g} / \mathrm{cm} \mathrm{s}^{2}\right)$ & $5.4829 \times 10^{5}$ & $5.4829 \times 10^{5}$ & 0 \\
$c_{s}(\mathrm{~cm} / \mathrm{s})$ & 32043 & 32043 & 0 \\
$T(\mathrm{~K})$ & 296 & 296 & 0 \\
$u(\mathrm{~cm} / \mathrm{s})$ & 0 & 0 & 0 \\
\hline \hline
\end{tabular}

\begin{tabular}{|c|c|c|c|}
\hline \hline \multicolumn{4}{|c|}{ Region 2 } \\
\hline \hline & Analytical values & WENO results & Error $(\%)$ \\
\hline$\rho\left(\mathrm{g} / \mathrm{cm}^{3}\right)$ & $2.95 \times 10^{-3}$ & $2.95 \times 10^{-3}$ & 0 \\
$p\left(\mathrm{~g} / \mathrm{cm} \mathrm{s}^{2}\right)$ & $5.4829 \times 10^{5}$ & $5.4829 \times 10^{5}$ & 0 \\
$c_{s}(\mathrm{~cm} / \mathrm{s})$ & 17600 & 17600 & 0 \\
$T(\mathrm{~K})$ & 293.5 & 293.5 & 0 \\
$u(\mathrm{~cm} / \mathrm{s})$ & 0 & 0 & 0 \\
\hline \hline
\end{tabular}

\begin{tabular}{|c|c|c|c|}
\hline \hline \multicolumn{4}{|c|}{ Region 3 } \\
\hline \hline & Analytical values & WENO results & Error $(\%)$ \\
\hline$\rho\left(\mathrm{g} / \mathrm{cm}^{3}\right)$ & $1.1546 \times 10^{-3}$ & $1.1545 \times 10^{-3}$ & 0.001 \\
$p\left(\mathrm{~g} / \mathrm{cm} \mathrm{s}^{2}\right)$ & $8.4985 \times 10^{5}$ & $8.4986 \times 10^{5}$ & 0.001 \\
$c_{s}(\mathrm{~cm} / \mathrm{s})$ & 35025 & 35025 & 0 \\
$T(\mathrm{~K})$ & 353.7 & 353.7 & 0 \\
$u(\mathrm{~cm} / \mathrm{s})$ & 8811.87 & 8814.1 & 0.03 \\
\hline \hline
\end{tabular}

\begin{tabular}{|c|c|c|c|}
\hline \hline \multicolumn{4}{|c|}{ Region 4 } \\
\hline \hline & Analytical values & WENO results & Error $(\%)$ \\
\hline$\rho\left(\mathrm{g} / \mathrm{cm}^{3}\right)$ & $4.1142 \times 10^{-3}$ & $4.1 \times 10^{-3}$ & 0.35 \\
$p\left(\mathrm{~g} / \mathrm{cm} \mathrm{s}^{2}\right)$ & $9.6356 \times 10^{5}$ & $9.598 \times 10^{5}$ & 0.39 \\
$c_{s}(\mathrm{~cm} / \mathrm{s})$ & 19757 & 19752 & 0.03 \\
$T(\mathrm{~K})$ & 369.2 & 369.7 & 0.14 \\
$u(\mathrm{~cm} / \mathrm{s})$ & 6263.11 & 6249.7 & 0.21 \\
\hline \hline
\end{tabular}

\begin{tabular}{|c|c|c|c|}
\hline \hline \multicolumn{4}{|c|}{ Region 5 } \\
\hline \hline & Analytical values & WENO results & Error $(\%)$ \\
\hline$\rho\left(\mathrm{g} / \mathrm{cm}^{3}\right)$ & $1.238 \times 10^{-3}$ & $1.241 \times 10^{-3}$ & 0.24 \\
$p\left(\mathrm{~g} / \mathrm{cm} \mathrm{s}^{2}\right)$ & $9.6356 \times 10^{5}$ & $9.598 \times 10^{5}$ & 0.39 \\
$c_{s}(\mathrm{~cm} / \mathrm{s})$ & 35967 & 35903 & 0.18 \\
$T(\mathrm{~K})$ & 370.6 & 371.6 & 0.27 \\
$u(\mathrm{~cm} / \mathrm{s})$ & 6263.11 & 6249.7 & 0.21 \\
\hline \hline
\end{tabular}

Table 3: Results computed analytically and from numerical simulation for a normal $M a=1.2$ shock refraction at a Ar-Xe interface in all of the five refraction regions. 
The above constitutes a single equation which can be solved for $p_{5}$. The value of $u_{5}$ is then obtained from $p_{5}$. Since a rarefaction wave does not increase the entropy from region 3 to region 5 , quantities in region 5 are determined using the isentropic relations

$$
\begin{aligned}
\rho_{5} & =\rho_{3}\left(\frac{p_{5}}{p_{3}}\right)^{\frac{1}{\gamma_{1}}} \\
T_{5} & =T_{3}\left(\frac{p_{5}}{p_{3}}\right)^{\frac{\gamma_{1}-1}{\gamma_{1}}} \\
c_{s, 5} & =\sqrt{\frac{\gamma_{1} p_{5}}{\rho_{5}}}
\end{aligned}
$$

Finally, quantities in region 4 are determined by first computing the transmitted shock Mach number $M a_{t}$ and then using the shock jump relations. The results of the above computations are presented in Table 5 and the shock information is presented in Table 4.

Images from the WENO simulations illustrating the Xe-Ar normal shock refraction are presented in Fig. 14. Presented are contours for the density, pressure, and velocity $u$ at time $t=0 \mathrm{~ms}$ showing the incident shock and at time $t=0.15 \mathrm{~ms}$ showing the reflected rarefaction wave and the transmitted shock. The figures at time $t=0 \mathrm{~ms}$ present the initial incident shock in xenon approaching the material interface separating the gases. Initially, the two gases are at rest and at the same pressures. Behind the shock, xenon is set in motion and the pressure is increased. The figures at $t=0.15 \mathrm{~ms}$ show that the reflected rarefaction wave further accelerates the flow and decreases the pressure from region 3 to region 5 . The pressures and the velocities are matched across the contact surface.

The density, pressure, and velocity in the different regions can be determined from the simulations. Next, the speeds of sound and the temperatures are computed. The quantities obtained from the simulation, together with the quantities determined analytically, are presented in Table 5. The analytical and numerical results are in excellent agreement.

\subsubsection{Oblique shock refraction at an Ar-Xe interface with a reflected shock}

Consider a contact surface separating argon and xenon, and forming an angle $\beta_{\text {interface }}=75^{\circ}$ with the plane of shock propagation moving at $M a_{s}=1.2$. Note that the shock and the interface form an angle $\beta_{i}=15^{\circ}$. Quantities in regions 1 and 2 are obtained from the initial data. Polar shock analysis is then used to determine the quantities in the other regions. Set $M a_{i}=M a_{s} / \sin \beta_{i}$. Specifying $p_{1}$, the shock polar corresponding to the initial incident shock can be represented in the $(\theta, p)$-plane. This shock polar is not needed to solve the oblique shock refraction problem but is included for completeness. The quantities in region 3 are immediately determined from the oblique shock relations. These relations also yield the flow turning angle for the initial incident shock $\theta_{i}$ and the flow Mach number in the region $3, M a_{r}$.

From the quantities in region 2 it is possible to determine the Mach number of the flow in region 2 when the flow is observed in a frame moving with the triple-point. This Mach number is referred to as $M a_{t}$ as it is the Mach number used to draw the shock polar for the transmitted shock. Note that the shock polar for the transmitted shock lies above the shock polar for the initial incident shock. Therefore, a reflected shock is needed to increase the pressure from $p_{3}$ to $p_{5}$. From the Mach number in region 3 it is possible to represent the shock polar in the $(\theta, p)$-plane corresponding to the reflected shock. The new shock polar is centered at the point $\left(\theta_{i}, p_{3}\right)$ as the flow has already been turned by the angle $\theta_{i}$ and the pressure has 


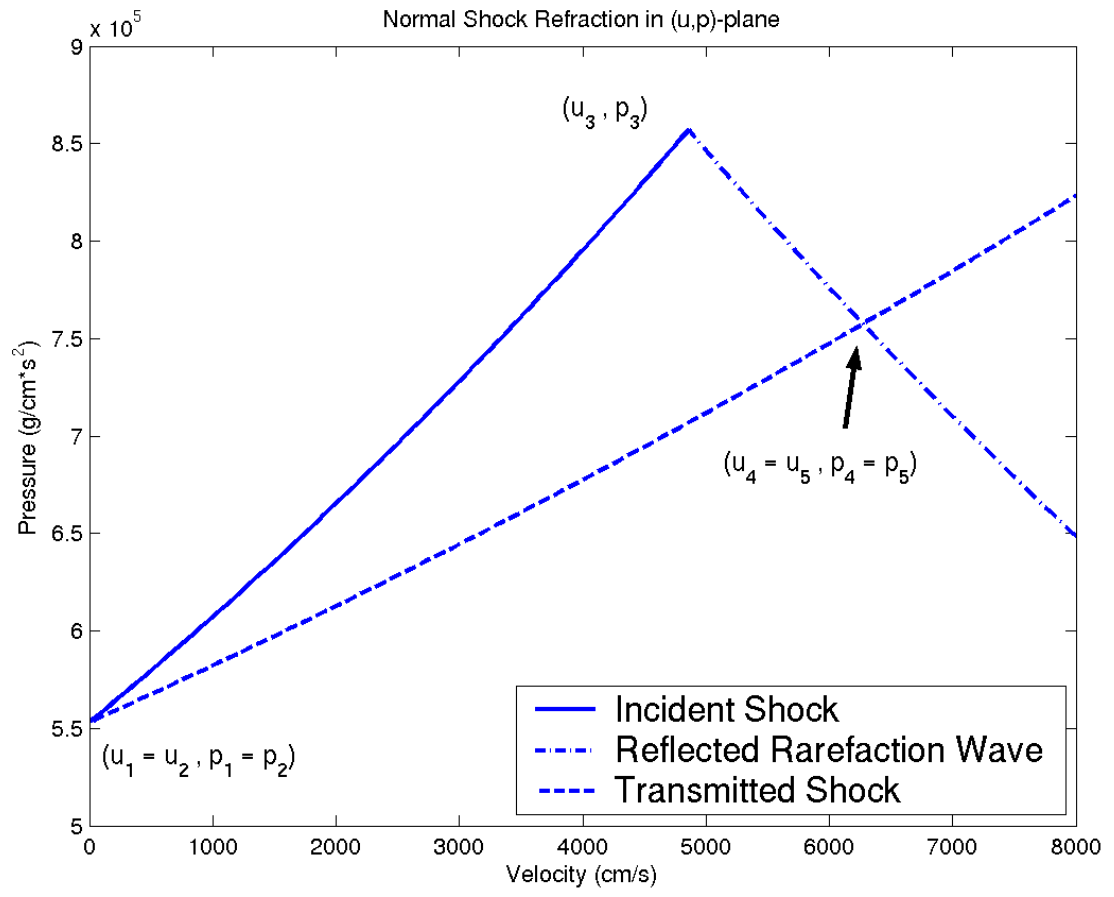

Figure 13: The refraction process observed for a $M a=1.2$ shock in Xe interacting with a contact surface separating $\mathrm{Xe}$ and $\mathrm{Ar}$ in the $(u, p)$-plane. The intersection of the reflected rarefaction wave with the transmitted shock curves constitutes the solution of the refraction process.

\begin{tabular}{|c|c|c|}
\hline & $M a$ & $u_{\text {shock }}=c_{s} M a(\mathrm{~cm} / \mathrm{s})$ \\
\hline \hline Incident shock & 1.2 & 21,210 \\
Transmitted shock & 1.1384 & 36,633 \\
\hline
\end{tabular}

Table 4: Results for the shock Mach numbers and the shock velocities in the normal Xe-Ar refraction. Note that the speed of the shock is increased following refraction, corresponding to a slow-fast refraction. 


$$
t=0 \mathrm{~ms}
$$
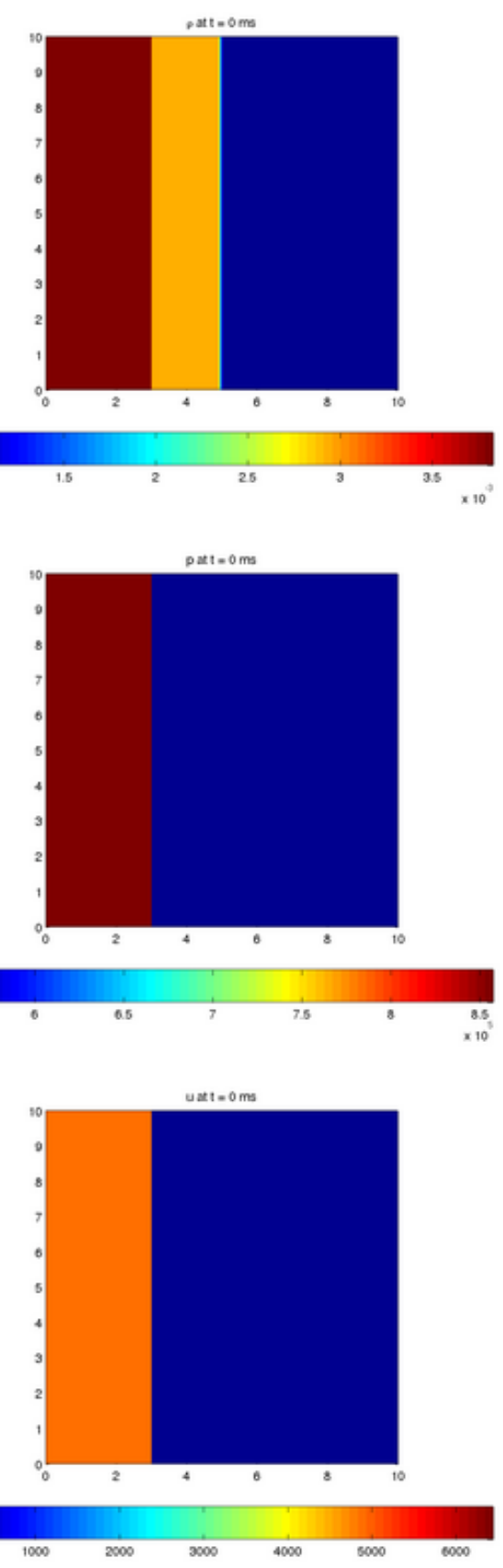

$t=0.15 \mathrm{~ms}$
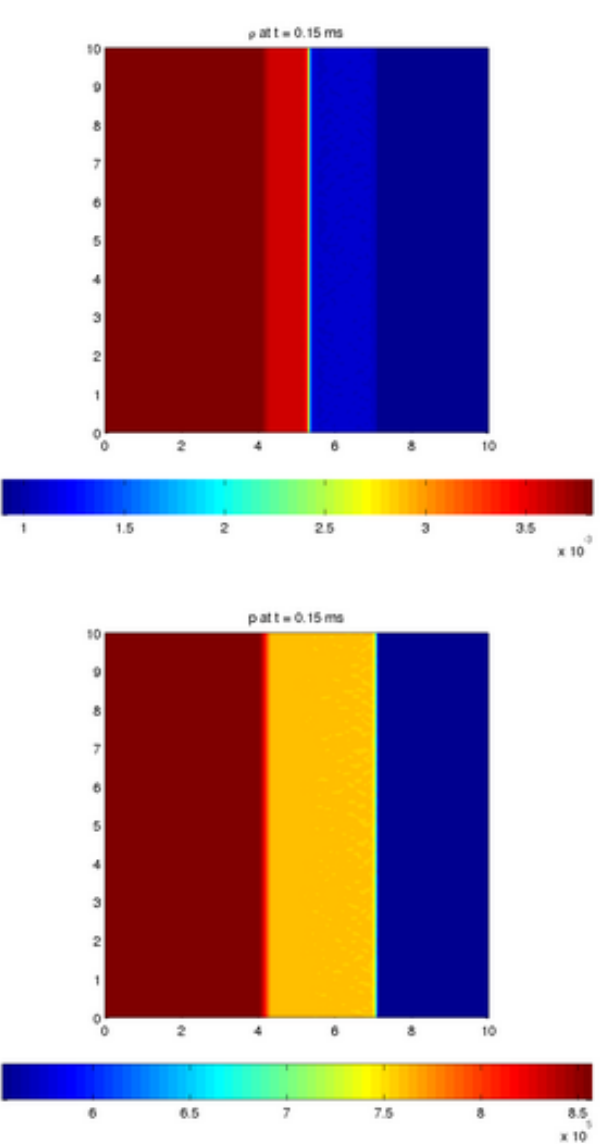

uatt $=0.15 \mathrm{~ms}$

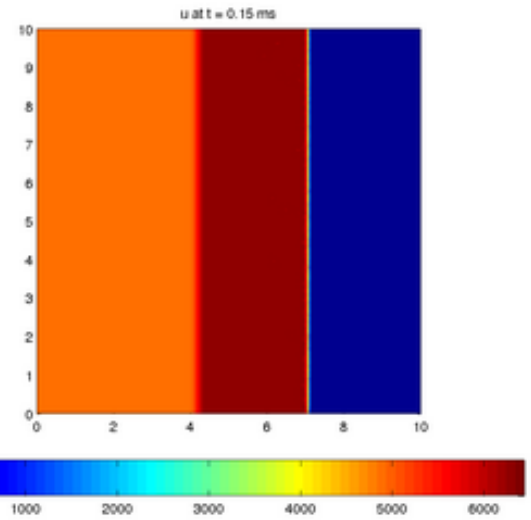

Figure 14: The density, pressure, and velocity $u$ at $t=0$ and $0.15 \mathrm{~ms}$ (right column) from a WENO simulation of the Xe-Ar shock refraction for a $M a=1.2$ shock. The figures at $t=0$ ms show the incident shock, while the figures at $t=0.15 \mathrm{~ms}$ show the reflected rarefaction wave and the transmitted shock wave. 


\begin{tabular}{|c|c|c|c|}
\hline \multicolumn{4}{|c|}{ Region 1 } \\
\hline \hline & Analytical values & WENO results & Error $(\%)$ \\
\hline$\rho\left(\mathrm{g} / \mathrm{cm}^{3}\right)$ & $2.95 \times 10^{-3}$ & $2.95 \times 10^{-3}$ & 0 \\
$p\left(\mathrm{~g} / \mathrm{cm} \mathrm{s}^{2}\right)$ & $5.5298 \times 10^{5}$ & $5.5298 \times 10^{5}$ & 0 \\
$c_{s}(\mathrm{~cm} / \mathrm{s})$ & 17675 & 17675 & 0 \\
$T(\mathrm{~K})$ & 296 & 296 & 0 \\
$u(\mathrm{~cm} / \mathrm{s})$ & 0 & 0 & 0 \\
\hline \hline
\end{tabular}

\begin{tabular}{|c|c|c|c|}
\hline \hline \multicolumn{4}{|c|}{ Region 2 } \\
\hline \hline & Analytical values & WENO results & Error $(\%)$ \\
\hline$\rho\left(\mathrm{g} / \mathrm{cm}^{3}\right)$ & $8.9 \times 10^{-4}$ & $8.9 \times 10^{-4}$ & 0 \\
$p\left(\mathrm{~g} / \mathrm{cm} \mathrm{s}^{2}\right)$ & $5.5298 \times 10^{5}$ & $5.5298 \times 10^{5}$ & 0 \\
$c_{s}(\mathrm{~cm} / \mathrm{s})$ & 32179 & 32179 & 0 \\
$T(\mathrm{~K})$ & 298.5 & 298.5 & 0 \\
$u(\mathrm{~cm} / \mathrm{s})$ & 0 & 0 & 0 \\
\hline \hline
\end{tabular}

\begin{tabular}{|c|c|c|c|}
\hline \hline \multicolumn{4}{|c|}{ Region 3 } \\
\hline \hline & Analytical values & WENO results & Error $(\%)$ \\
\hline$\rho\left(\mathrm{g} / \mathrm{cm}^{3}\right)$ & $3.827 \times 10^{-3}$ & $3.827 \times 10^{-3}$ & 0 \\
$p\left(\mathrm{~g} / \mathrm{cm} \mathrm{s}^{2}\right)$ & $8.5711 \times 10^{5}$ & $8.5713 \times 10^{5}$ & 0.001 \\
$c_{s}(\mathrm{~cm} / \mathrm{s})$ & 19320 & 19320 & 0 \\
$T(\mathrm{~K})$ & 353.7 & 353.7 & 0 \\
$u(\mathrm{~cm} / \mathrm{s})$ & 4860.7 & 4860.7 & 0 \\
\hline \hline
\end{tabular}

\begin{tabular}{|c|c|c|c|}
\hline \hline \multicolumn{4}{|c|}{ Region 4 } \\
\hline \hline & Analytical values & WENO results & Error $(\%)$ \\
\hline$\rho\left(\mathrm{g} / \mathrm{cm}^{3}\right)$ & $1.1055 \times 10^{-3}$ & $1.0737 \times 10^{-3}$ & 2.88 \\
$p\left(\mathrm{~g} / \mathrm{cm} \mathrm{s}^{2}\right)$ & $7.5757 \times 10^{5}$ & $7.5754 \times 10^{5}$ & 0.01 \\
$c_{s}(\mathrm{~cm} / \mathrm{s})$ & 33794 & 34291 & 1.45 \\
$T(\mathrm{~K})$ & 338.9 & 339 & 0.03 \\
$u(\mathrm{~cm} / \mathrm{s})$ & 6274.5 & 6265.1 & 0.15 \\
\hline \hline
\end{tabular}

\begin{tabular}{|c|c|c|c|}
\hline \hline \multicolumn{4}{|c|}{ Region 5 } \\
\hline \hline & Analytical values & WENO results & Error $(\%)$ \\
\hline$\rho\left(\mathrm{g} / \mathrm{cm}^{3}\right)$ & $3.553 \times 10^{-3}$ & $3.554 \times 10^{-3}$ & 0.03 \\
$p\left(\mathrm{~g} / \mathrm{cm} \mathrm{s}^{2}\right)$ & $7.5757 \times 10^{5}$ & $7.5754 \times 10^{5}$ & 0.01 \\
$c_{s}(\mathrm{~cm} / \mathrm{s})$ & 18849 & 18848 & 0.01 \\
$T(\mathrm{~K})$ & 336.6 & 336.6 & 0 \\
$u(\mathrm{~cm} / \mathrm{s})$ & 6276 & 6265 & 0.03 \\
\hline \hline
\end{tabular}

Table 5: Results computed analytically and obtained from the WENO simulation for an oblique $M a=1.2$ shock refraction at a Xe-Ar interface. 


\begin{tabular}{|c|c|c|c|}
\hline & $M a$ & $\beta\left(^{\circ}\right)$ & $\theta\left(^{\circ}\right)$ \\
\hline \hline Incident shock & 4.6364 & 15 & 3.33 \\
Reflected shock & 4.1836 & 165.88 & -0.94 \\
Transmitted shock & 8.4411 & 8.61 & 2.39 \\
\hline
\end{tabular}

Table 6: Shock Mach numbers, shock-interface angle $\beta$, and flow turning angles $\theta$ for the oblique Xe-Ar refraction determined analytically.

already increased to $p_{3}$. The intersection of the reflected and the transmitted shock polars in the $(\theta, p)$-plane constitute solutions of the refraction process. The pressure following the reflected and the transmitted shock is matched at these points, and the flow turning angles satisfy the relation (25).

Figure 15 shows a plot of the three shock polars corresponding to the incident, reflected, and transmitted shocks. Note that there are several solutions to the oblique shock refraction problem. In general, the solution observed in experiments and in numerical simulations is the solution with the smallest change in pressure. Therefore, the desired solution is found in the lower part of Fig. 15: a close-up of the region is shown in Fig. 16. Once the solution state has been identified in Fig. 16, the shock angles $\beta_{r}$ and $\beta_{t}$ that yield that point on both the reflected and the transmitted shock polar are determined. This is accomplished iteratively by adjusting the quantities in order to obtain matching pressures and flow turning angles that satisfy relation (25). The solution of the refraction problem in all the five regions together with numerical results is presented in Table 7 and the shock results are presented in Table 6 .

Images from the WENO simulations illustrating the Ar-Xe oblique shock refraction are presented in Fig. 17. Presented are contour plots for the density, pressure, and horizontal and vertical velocity $u$ and $v$. As all waves coincide at the triple-point, only plots at $t=0.12$ $\mathrm{ms}$ are presented to illustrate all five refraction regions. In particular, the density plots show all five regions. Note that the the transmitted shock and the incident shock form an angle greater than $180^{\circ}$. This is due to the fact that the transmitted shock now moves slower than the incident shock; Henderson refers to this as a fast-slow refraction. The pressure plot shows that the matching conditions are satisfied with $p_{1}=p_{2}$ and $p_{4}=p_{5}$. Finally, the plots of the horizontal and vertical velocity reveal that there is a slight discrepancy in the velocities between region 4 and region 5 . This is to be expected as the matching conditions only impose that the normal component be matched. From the plot of the vertical velocity $v$ it may be inferred that a shear forms at the material interface as the tangential velocities are different. The numerical and analytical results are presented in Table 7. The analytical and numerical results are in excellent agreement.

\subsubsection{Oblique shock refraction at an Xe-Ar interface with a reflected rarefaction wave}

Consider now a contact surface separating xenon and argon forming an angle $\beta_{\text {inter face }}=75^{\circ}$ with the plane of propagation of the $M a_{s}=1.2$ shock corresponding to $\beta_{i}=15^{\circ}$. All quantities in regions 1 and 2 can be determined from the initial data. Set $M a_{i}=M a_{s} / \sin \beta_{i}$ and draw the shock polar for the incident shock in the $(\theta, p)$-plane. This shock polar is not needed for the refraction solution, but is included for completeness. The quantities in region 3 are determined from the oblique shock relations. Next, determine the Mach number of the flow in region 2 


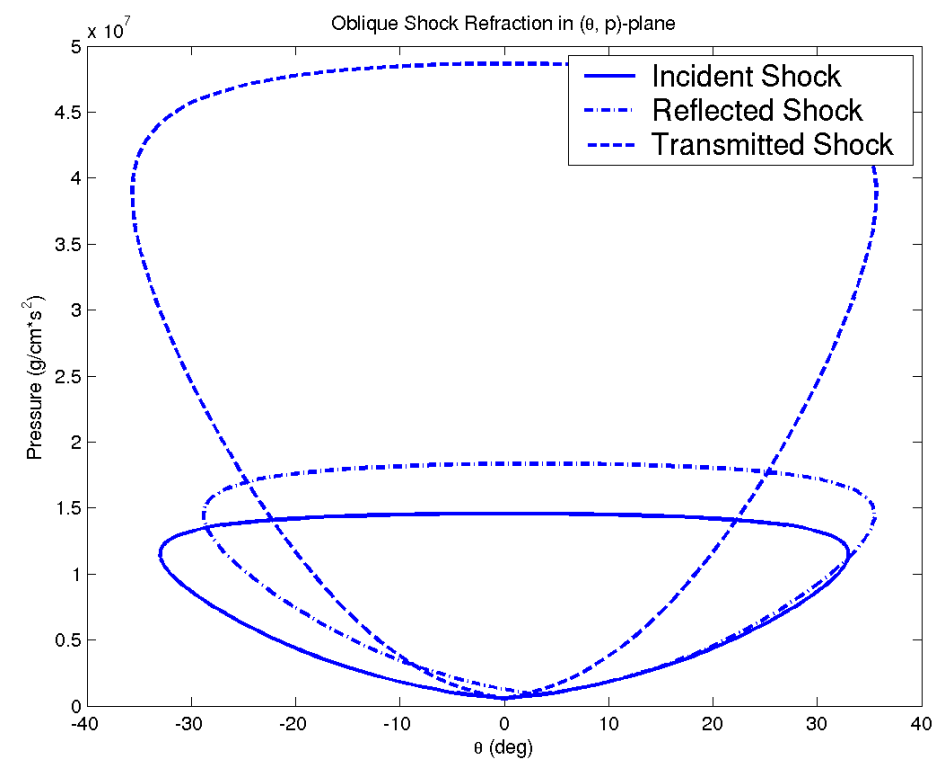

Figure 15: The shock polars for the incident, reflected, and transmitted shocks in the $(\theta, p)$ plane for Ar-Xe oblique shock refraction. Note that the transmitted shock polar originates right on top of the incident shock polar, but shifted by the amount $\theta_{i}$ and $p_{3}$ corresponding to the state behind the incident shock. The points where the reflected shock polar and the transmitted shock polar intersect correspond to the solution of the refraction problem. At those points the pressure $p_{5}=p_{4}$ is matched and the flow turning angles satisfy $\theta_{t}=\theta_{i}+\theta_{r}$.

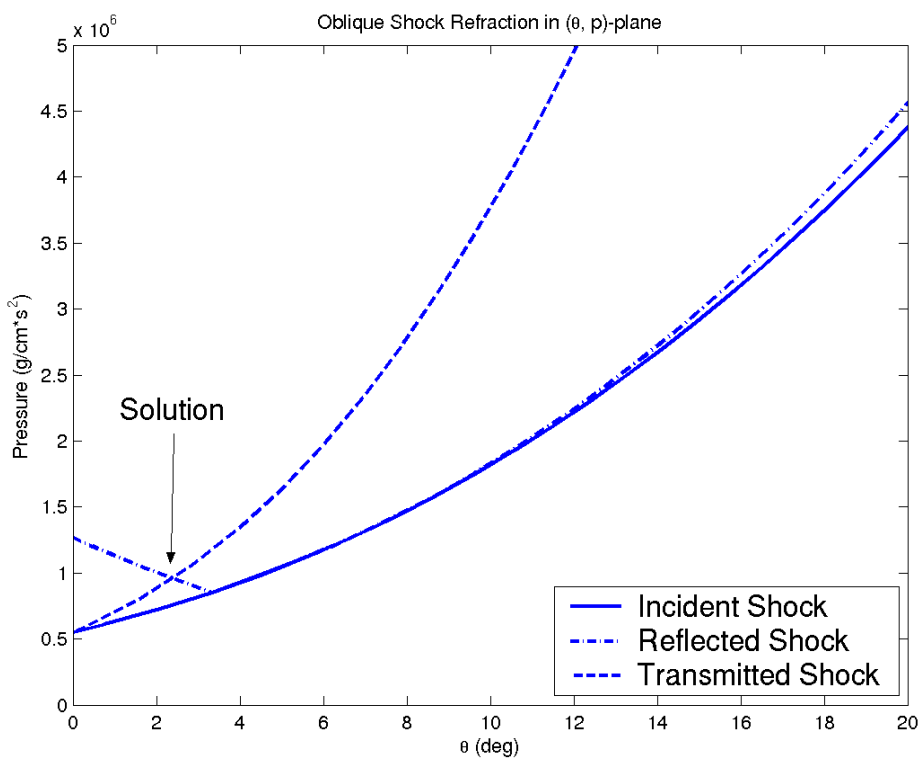

Figure 16: A close up of Fig. 15 for the solution with the smallest change in pressure. 

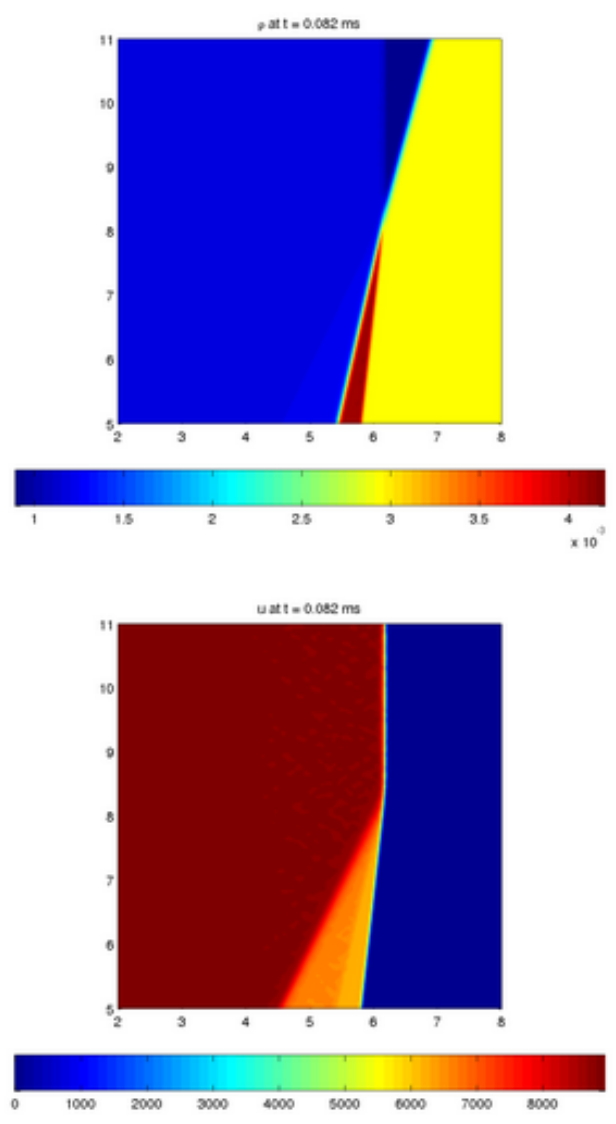
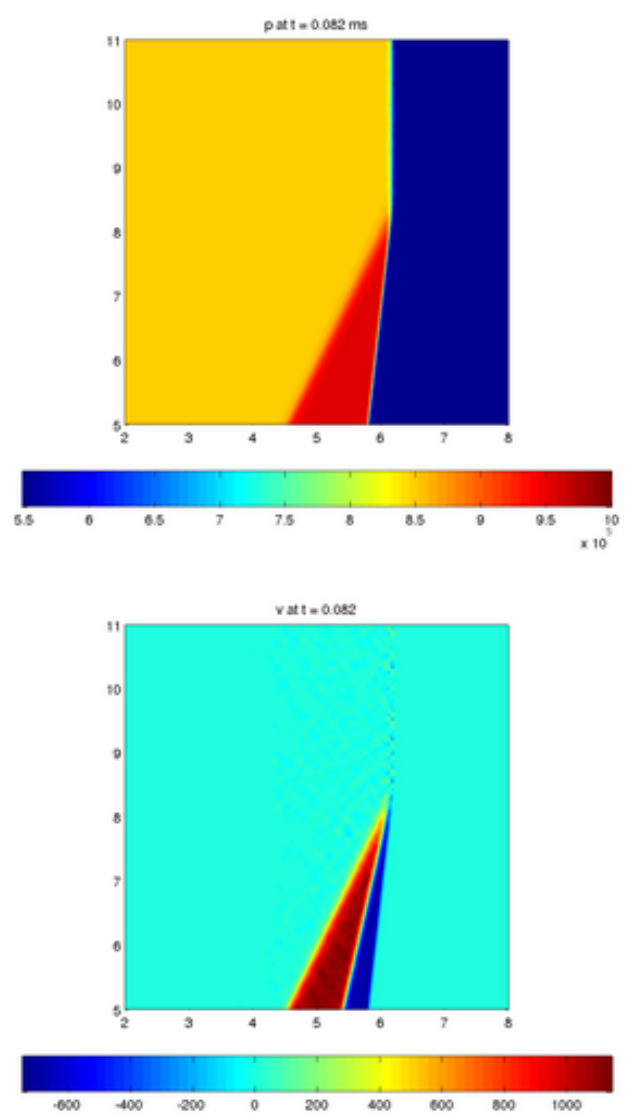

Figure 17: The density, pressure, horizontal velocity $u$, and vertical velocity $v$ at $t=0.082$ ms from a WENO simulation of an oblique $M a=1.2$ shock refraction at an Ar-Xe interface when the angle between the interface and the plane of motion of the shock is $\beta=75^{\circ}$. All of the waves coincide at the triple-point, affording a view of all five refraction regions. Note the reflected shock wave following refraction. 


\begin{tabular}{|c|c|c|c|}
\hline \multicolumn{4}{|c|}{ Region 1 } \\
\hline \hline & Analytical values & WENO results & Error $(\%)$ \\
\hline$\rho\left(\mathrm{g} / \mathrm{cm}^{3}\right)$ & $8.9 \times 10^{-4}$ & $8.9 \times 10^{-4}$ & 0 \\
$p\left(\mathrm{~g} / \mathrm{cm} \mathrm{s}^{2}\right)$ & $5.4829 \times 10^{5}$ & $5.4829 \times 10^{5}$ & 0 \\
$c_{s}(\mathrm{~cm} / \mathrm{s})$ & 32043 & 32043 & 0 \\
$T(\mathrm{~K})$ & 296 & 296 & 0 \\
$u(\mathrm{~cm} / \mathrm{s})$ & & 0 & \\
$v(\mathrm{~cm} / \mathrm{s})$ & & 0 & \\
\hline \hline
\end{tabular}

\begin{tabular}{|c|c|c|c|}
\hline \hline \multicolumn{4}{|c|}{ Region 2 } \\
\hline \hline & Analytical values & WENO results & Error $(\%)$ \\
\hline$\rho\left(\mathrm{g} / \mathrm{cm}^{3}\right)$ & $2.95 \times 10^{-3}$ & $2.95 \times 10^{-3}$ & 0 \\
$p\left(\mathrm{~g} / \mathrm{cm} \mathrm{s}^{2}\right)$ & $5.4829 \times 10^{5}$ & $5.4829 \times 10^{5}$ & 0 \\
$c_{s}(\mathrm{~cm} / \mathrm{s})$ & 17600 & 17600 & 0 \\
$T(\mathrm{~K})$ & 293.5 & 293.5 & 0 \\
$u(\mathrm{~cm} / \mathrm{s})$ & & 0 & \\
$v(\mathrm{~cm} / \mathrm{s})$ & & 0 & \\
\hline \hline
\end{tabular}

\begin{tabular}{|c|c|c|c|}
\hline \hline \multicolumn{4}{|c|}{ Region 3 } \\
\hline \hline & Analytical values & WENO results & Error $(\%)$ \\
\hline$\rho\left(\mathrm{g} / \mathrm{cm}^{3}\right)$ & $1.1546 \times 10^{-3}$ & $1.1546 \times 10^{-3}$ & 0 \\
$p\left(\mathrm{~g} / \mathrm{cm} \mathrm{s}^{2}\right)$ & $8.4985 \times 10^{5}$ & $8.4986 \times 10^{5}$ & 0.001 \\
$c_{s}(\mathrm{~cm} / \mathrm{s})$ & 35025 & 35025 & 0 \\
$T(\mathrm{~K})$ & 353.7 & 353.7 & 0 \\
$u(\mathrm{~cm} / \mathrm{s})$ & & 8937.8 & \\
$v(\mathrm{~cm} / \mathrm{s})$ & & 0 & \\
\hline \hline
\end{tabular}

\begin{tabular}{|c|c|c|c|}
\hline \hline \multicolumn{4}{|c|}{ Region 4 } \\
\hline \hline$\rho\left(\mathrm{g} / \mathrm{cm}^{3}\right)$ & Analytical values & WENO results & Error $(\%)$ \\
$p\left(\mathrm{~g} / \mathrm{cm} \mathrm{s}^{2}\right)$ & $9.099 \times 10^{-3}$ & $4.202 \times 10^{-3}$ & 2.45 \\
$c_{s}(\mathrm{~cm} / \mathrm{s})$ & 19730 & $1.0004 \times 10^{5}$ & 4.3 \\
$T(\mathrm{~K})$ & 369 & 19920 & 0.95 \\
$u(\mathrm{~cm} / \mathrm{s})$ & & 376 & 1.86 \\
$v(\mathrm{~cm} / \mathrm{s})$ & & 6173.1 & \\
\hline \hline
\end{tabular}

\begin{tabular}{|c|c|c|c|}
\hline \hline \multicolumn{4}{|c|}{ Region 5 } \\
\hline & Analytical values & WENO results & Error $(\%)$ \\
\hline \hline$\rho\left(\mathrm{g} / \mathrm{cm}^{3}\right)$ & $1.249 \times 10^{-3}$ & $1.2388 \times 10^{-3}$ & 0.82 \\
$p\left(\mathrm{~g} / \mathrm{cm} \mathrm{s}^{2}\right)$ & $9.551 \times 10^{5}$ & $1.0004 \times 10^{6}$ & 4.87 \\
$c_{s}(\mathrm{~cm} / \mathrm{s})$ & 35853 & 36887 & 2.75 \\
$T(\mathrm{~K})$ & 371 & 388 & 1.8 \\
$u(\mathrm{~cm} / \mathrm{s})$ & & 6578.1 & \\
$v(\mathrm{~cm} / \mathrm{s})$ & & -691.2 & \\
\hline \hline
\end{tabular}

Table 7: Results computed analytically and from numerical simulation for an oblique $M a=1.2$ shock refraction at a Ar-Xe interface with $\beta_{\text {interface }}=75^{\circ}$. Note the excellent agreement between the analytical and the numerical results. 


\begin{tabular}{|c|c|c|c|}
\hline & $M a$ & $\beta\left(^{\circ}\right)$ & $\theta\left(^{\circ}\right)$ \\
\hline \hline Incident shock & 4.6364 & 15 & 3.33 \\
Reflected rarefaction wave & & & 0.89 \\
Transmitted shock & 2.5466 & 26.68 & 4.2 \\
\hline
\end{tabular}

Table 8: Shock Mach numbers for oblique Xe-Ar refraction determined analytically.

\begin{tabular}{|c|c|c|}
\hline & $M a$ & $\Theta(M a)\left(^{\circ}\right)$ \\
\hline \hline Region 3 & 4.1836 & 51.4048 \\
Region 5 & 4.2958 & 52.2982 \\
\hline
\end{tabular}

Table 9: Flow Mach numbers ahead of and behind the reflected rarefaction wave, and the values of the Prandtl-Meyer angles for the oblique Xe-Ar refraction.

when the flow is observed in a reference frame moving with the triple-point. This Mach number $M a_{t}=M a_{i} c_{s, 1} / c_{s, 2}$ is used to draw the shock polar corresponding to the transmitted shock. The shock polar for the transmitted shock lies below the shock polar of the incident shock. More specifically, the point $\left(\theta_{i}, p_{3}\right)$ lies above the transmitted shock. Therefore, a reflected shock cannot form as such a shock further increases the pressure from $p_{3}$ to $p_{5}$; instead, a reflected rarefaction wave forms to decrease the pressure.

The pressure and the flow turning angle in a reflected rarefaction wave are related through the Prandtl-Meyer function $\Theta(M a)$ as derived in Eq. (31) and through Eq. (36). It is natural to consider $\theta_{r}$ as the parameter for the curve in the $(\theta, p)$-plane and express $p_{5}=p_{5}\left(\theta_{r}\right)$. Unfortunately, this approach is quite difficult as the Prandtl-Meyer function must be inverted, and this can only be accomplished with great difficulty. However, there is an equivalent formulation in which $M a_{5}$ is the parameter, $\theta_{r}=\theta_{r}\left(M a_{5}\right)$ is determined with the PrandtlMeyer function and $p_{5}=p_{5}\left(M a_{5}\right)$ with the isentropic flow relations. This is the procedure followed in the present investigation. Note that the smallest value for $M a_{5}$ is $M a_{3}$, as the Mach number is increased after a reflected rarefaction wave. However, for the purpose of illustration in Fig. 18, values smaller than $M a_{3}$ are considered. Furthermore, note that when the curve is drawn in the same $(\theta, p)$-plane as the incident and transmitted shock polars, the curve must be shifted by $\theta_{i}$ as the flow has already been rotated by the angle $\theta_{i}$ by the incident shock.

After all three curves are drawn on the $(\theta, p)$-plane, the intersection between the reflected rarefaction wave and the transmitted shock polar constitute the solution to the refraction problem. Thus, the Mach number $M a_{5}$ that gives the desired point on the reflected rarefaction wave is determined iteratively. Quantities in region 5 can be determined using the value of $M a_{5}$. The shock angle $\beta_{t}$ that gives the same point on the transmitted shock is determined, and all quantities in region 4 are then determined via the oblique shock relations. The results of the Xe-Ar refraction problem in all five regions are presented in Table 10.

Figure 20 shows images from the WENO simulations illustrating the Xe-Ar oblique shock refraction. Presented are contour plots for the density, pressure, and horizontal and vertical velocity at $t=0.1 \mathrm{~ms}$. In particular, the density plot distinguishes between all five regions. In the upper part of the plot, the incident shock refracts at the oblique interface, and the transmitted shock and the reflected rarefaction wave in the lower part. The transmitted shock wave now forms an angle with the initial incident shock that is smaller than $180^{\circ}$. This is due 


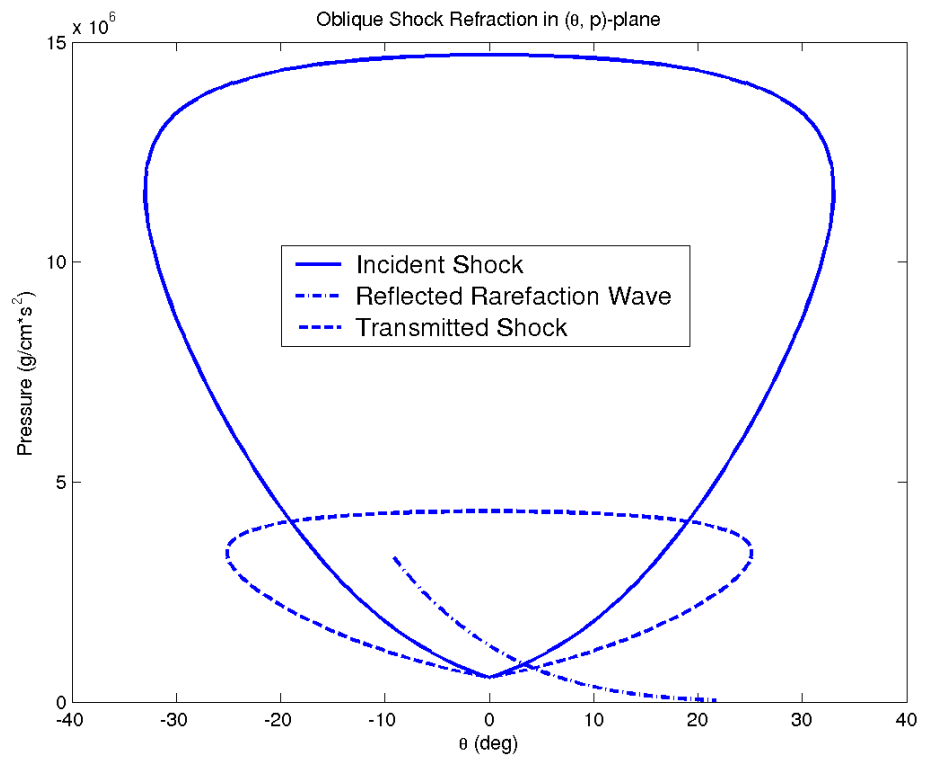

Figure 18: Shock polars for the incident, reflected, and transmitted shocks for Xe-Ar oblique shock refraction observed in the $(\theta, p)$-plane. Note that the transmitted shock polar originates right on top of the incident shock polar, but shifted by the amount $\theta_{i}$ and $p_{3}$. The points where the reflected shock polar and the transmitted shock polar intersect correspond to the solution of the refraction problem. At those points the pressure $p_{5}=p_{4}$ is matched and the flow turning angles satisfy $\theta_{t}=\theta_{i}+\theta_{r}$.

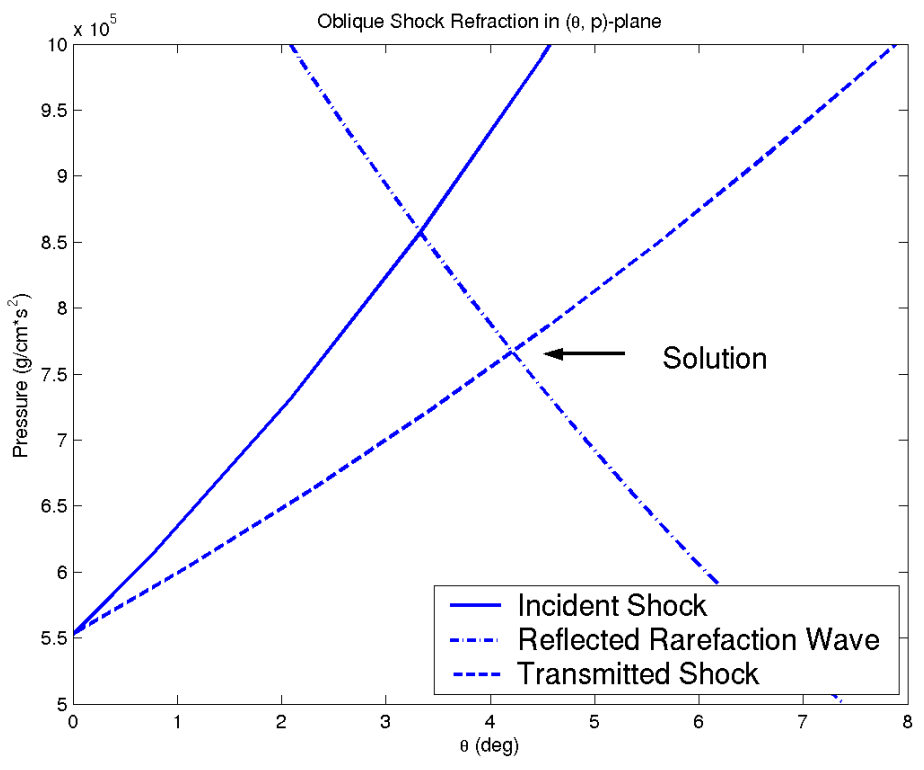

Figure 19: A close up of Fig. 18. The intersection of the oblique shock polar and of the transmitted shock polar constitutes the solution of the refraction process. 
to the fact that the transmitted shock moves faster in the second gas than in the first gas; Henderson refers to this as slow-fast refraction. This is in contrast to the Ar-Xe oblique shock refraction of Fig. 17. The pressure plot shows that the pressure is matched across regions 1 and 2 and across regions 4 and 5 . The pressure in region 5 is lower than the pressure in region 3 following the rarefaction wave. The $u$ and $v$ plots show a mismatch across regions 4 and 5 following refraction. This mismatch is expected as only the component of the velocity normal to the interface must match at a material interface. In particular, the jump in velocity observed in the $v$ plot reveals the presence of shear. Note that the direction of the shear is opposite to what observed in Fig. 17. The numerically computed results, together with the analytical results for the five regions, are presented in Table 10. The analytical and numerical results are in excellent agreement. 

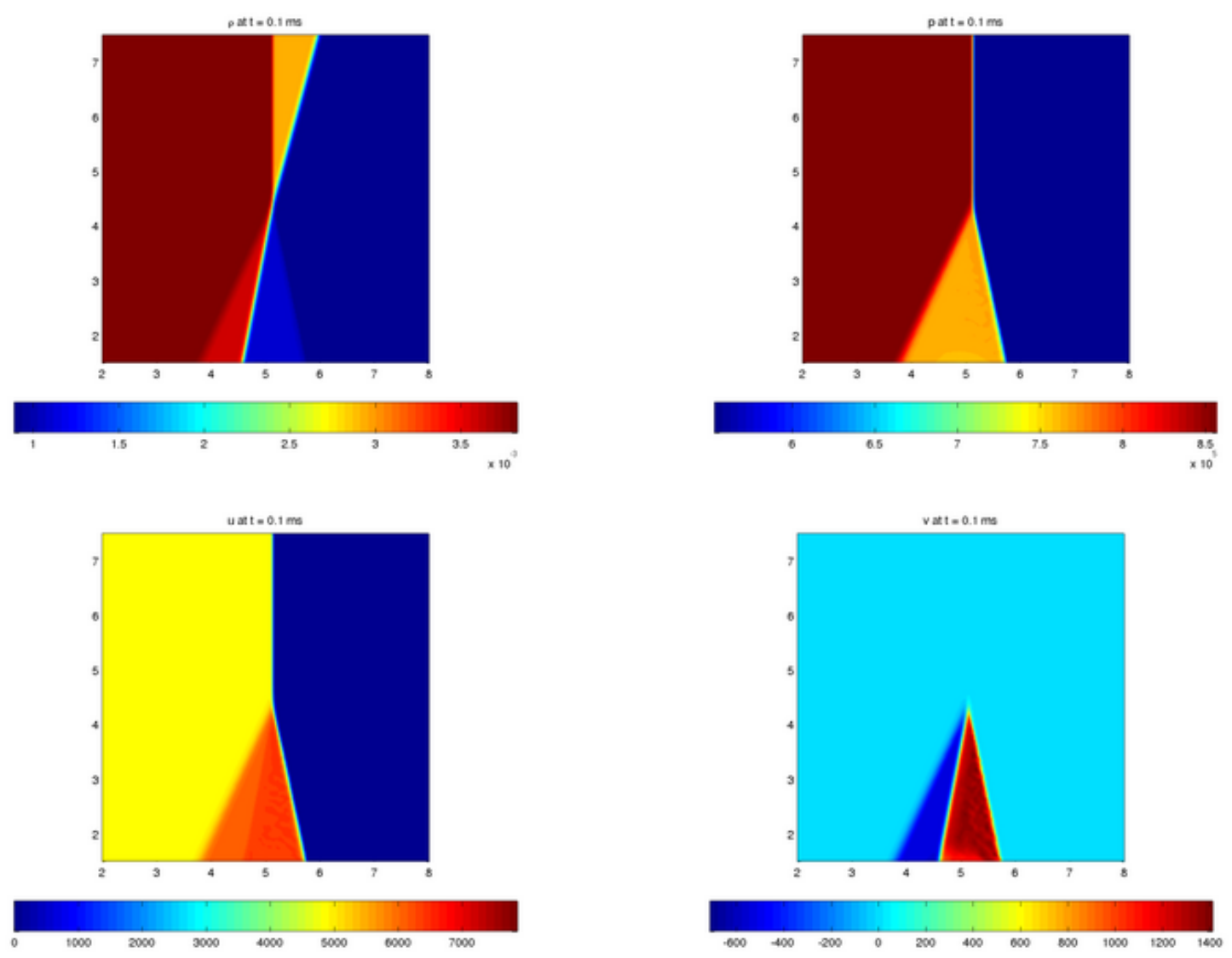

Figure 20: The density, pressure, horizontal velocity $u$, and vertical velocity $v$ at $t=0.1 \mathrm{~ms}$ from a WENO simulation of a $M a=1.2 \beta=75^{\circ}$ shock refracting on a Xe-Ar interface. All of the waves coincide at the triple-point. 


\begin{tabular}{|c|c|c|c|}
\hline \multicolumn{4}{|c|}{ Region 1 } \\
\hline \hline & Analytical values & WENO results & Error $(\%)$ \\
\hline$\rho\left(\mathrm{g} / \mathrm{cm}^{3}\right)$ & $2.95 \times 10^{-4}$ & $2.95 \times 10^{-4}$ & 0 \\
$p\left(\mathrm{~g} / \mathrm{cm} \mathrm{s}^{2}\right)$ & $5.5298 \times 10^{5}$ & $5.5298 \times 10^{5}$ & 0 \\
$c_{s}(\mathrm{~cm} / \mathrm{s})$ & 17675 & 17675 & 0 \\
$T(\mathrm{~K})$ & 296 & 296 & 0 \\
$u(\mathrm{~cm} / \mathrm{s})$ & & 0 & \\
$v(\mathrm{~cm} / \mathrm{s})$ & & 0 & \\
\hline \hline
\end{tabular}

\begin{tabular}{|c|c|c|c|}
\hline \hline \multicolumn{4}{|c|}{ Region 2 } \\
\hline \hline & Analytical values & WENO results & Error $(\%)$ \\
\hline$\rho\left(\mathrm{g} / \mathrm{cm}^{3}\right)$ & $8.9 \times 10^{-4}$ & $8.9 \times 10^{-4}$ & 0 \\
$p\left(\mathrm{~g} / \mathrm{cm} \mathrm{s}^{2}\right)$ & $5.5298 \times 10^{5}$ & $5.5298 \times 10^{5}$ & 0 \\
$c_{s}(\mathrm{~cm} / \mathrm{s})$ & 32179 & 32179 & 0 \\
$T(\mathrm{~K})$ & 298.5 & 298.5 & 0 \\
$u(\mathrm{~cm} / \mathrm{s})$ & & 0 & \\
$v(\mathrm{~cm} / \mathrm{s})$ & & 0 & \\
\hline \hline
\end{tabular}

\begin{tabular}{|c|c|c|c|}
\hline \hline \multicolumn{4}{|c|}{ Region 3 } \\
\hline \hline & Analytical values & WENO results & Error $(\%)$ \\
\hline \hline$\left(\mathrm{g} / \mathrm{cm}^{3}\right)$ & $3.827 \times 10^{-3}$ & $3.827 \times 10^{-3}$ & 0 \\
$p\left(\mathrm{~g} / \mathrm{cm} \mathrm{s}^{2}\right)$ & $8.5711 \times 10^{5}$ & $8.5713 \times 10^{5}$ & 0.001 \\
$c_{s}(\mathrm{~cm} / \mathrm{s})$ & 19320 & 19320 & 0 \\
$T(\mathrm{~K})$ & 353.7 & 353.7 & 0 \\
$u(\mathrm{~cm} / \mathrm{s})$ & & 4860.9 & \\
$v(\mathrm{~cm} / \mathrm{s})$ & & 0 & \\
\hline \hline
\end{tabular}

\begin{tabular}{|c|c|c|c|}
\hline \hline \multicolumn{4}{|c|}{ Region 4 } \\
\hline \hline & Analytical values & WENO results & Error $(\%)$ \\
\hline$\rho\left(\mathrm{g} / \mathrm{cm}^{3}\right)$ & $1.0806 \times 10^{-3}$ & $1.081 \times 10^{-3}$ & 0.04 \\
$p\left(\mathrm{~g} / \mathrm{cm} \mathrm{s}^{2}\right)$ & $7.655 \times 10^{5}$ & $7.6634 \times 10^{5}$ & 0.11 \\
$c_{s}(\mathrm{~cm} / \mathrm{s})$ & 34361 & 34373 & 0.04 \\
$T(\mathrm{~K})$ & 340.4 & 340.6 & 0.06 \\
$u(\mathrm{~cm} / \mathrm{s})$ & & 6341 & \\
$v(\mathrm{~cm} / \mathrm{s})$ & & 1322.5 & \\
\hline \hline
\end{tabular}

\begin{tabular}{|c|c|c|c|}
\hline \hline \multicolumn{4}{c|}{ Region 5 } \\
\hline \hline & Analytical values & WENO results & Error $(\%)$ \\
\hline$\rho\left(\mathrm{g} / \mathrm{cm}^{3}\right)$ & $3.5754 \times 10^{-3}$ & $3.577 \times 10^{-3}$ & 0.08 \\
$p\left(\mathrm{~g} / \mathrm{cm} \mathrm{s}^{2}\right)$ & $7.6525 \times 10^{5}$ & $7.6634 \times 10^{5}$ & 0.14 \\
$c_{s}(\mathrm{~cm} / \mathrm{s})$ & 18887 & 18896 & 0.05 \\
$T(\mathrm{~K})$ & 338 & 338.3 & 0.9 \\
$u(\mathrm{~cm} / \mathrm{s})$ & & 6027.4 & \\
$v(\mathrm{~cm} / \mathrm{s})$ & & -541 & \\
\hline \hline
\end{tabular}

Table 10: Results computed analytically and from numerical simulation for an oblique $M a=$ 1.2 shock refraction at a Xe-Ar interface with $\beta_{\text {interface }}=75^{\circ}$. 


\subsection{Refraction with different adiabatic exponents: the case of air(acetone) and $\mathrm{SF}_{6}$}

Now consider shock refraction at an air(acetone)-sulfur hexafluoride $\left(\mathrm{SF}_{6}\right)$ interface. The two gases have different adiabatic exponents and are selected to validate the HOPE code with gamma-blending.

\subsubsection{Normal shock refraction at an air(acetone)- $\mathrm{SF}_{6}$ interface with a reflected shock wave}

First consider the case of a $M a=1.2$ normal shock in air(acetone) refracting into $\mathrm{SF}_{6}$. All quantities in the five refraction regions are analytically determined from the initial conditions with the same procedure used for the Ar-Xe case in $\S$ 4.3.1. The shock polar for this case is presented in Fig 21. Images from the WENO simulation are shown in Fig. 22. Contour plots for the density, pressure, and velocity are presented at the initial time $t=0 \mathrm{~ms}$ showing the incident shock and at time $t=0.15 \mathrm{~ms}$ showing the reflected and transmitted shocks.

Numerical and analytical results can be compared in Table 12. The results for the shock Mach numbers are presented in Table 11. The analytical and numerical results are in excellent agreement.

\subsubsection{Normal shock refraction at an $\mathrm{SF}_{6}$-air(acetone) interface with a reflected rarefaction wave}

Now consider a normal $M a=1.21$ shock in $\mathrm{SF}_{6}$ refracting into air(acetone). All quantities in the five refraction region are analytically determined from the initial conditions with the same procedure used for the Xe-Ar case of $\S$ 4.3.2. The shock polar is presented in Fig. 23. Images from the WENO simulations are shown in Fig. 24. Contour plots for the density, pressure, and velocity are presented at the initial time $t=0 \mathrm{~ms}$ showing the incident shock and at time $t=0.15 \mathrm{~ms}$ showing the reflected rarefaction wave and transmitted shock. Numerical and analytical results are compared in Table 14. The analytical results for the shocks and the rarefaction wave are presented in Table 13 . The analytical and numerical results are in excellent agreement.

\subsubsection{Oblique shock refraction at an air(acetone)-SF $\mathrm{S}_{6}$ interface with a reflected shock}

Next, consider a contact surface separating air(acetone) and $\mathrm{SF}_{6}$ forming an angle $\beta_{\text {interface }}=$ $75^{\circ}$ with the plane of propagation of the shock. Note that the shock and the interface form an angle $\beta_{i}=15^{\circ}$. All quantities in the five refraction regions are analytically determined with the same procedure used for the Ar-Xe oblique refraction in $\S$ 4.3.3. The shock polar is presented in Fig. 25 with a close up in Fig. 26. Results for the shock interface angles $\beta$ and flow turning angles $\theta$ are presented in Table 16. Images from the WENO simulations are shown in Fig. 27. Presented are contour plots of the density, pressure, and horizontal and vertical velocity at $t=0.12 \mathrm{~ms}$. The analytical and numerical results are compared in Table 16 and are in excellent agreement. 


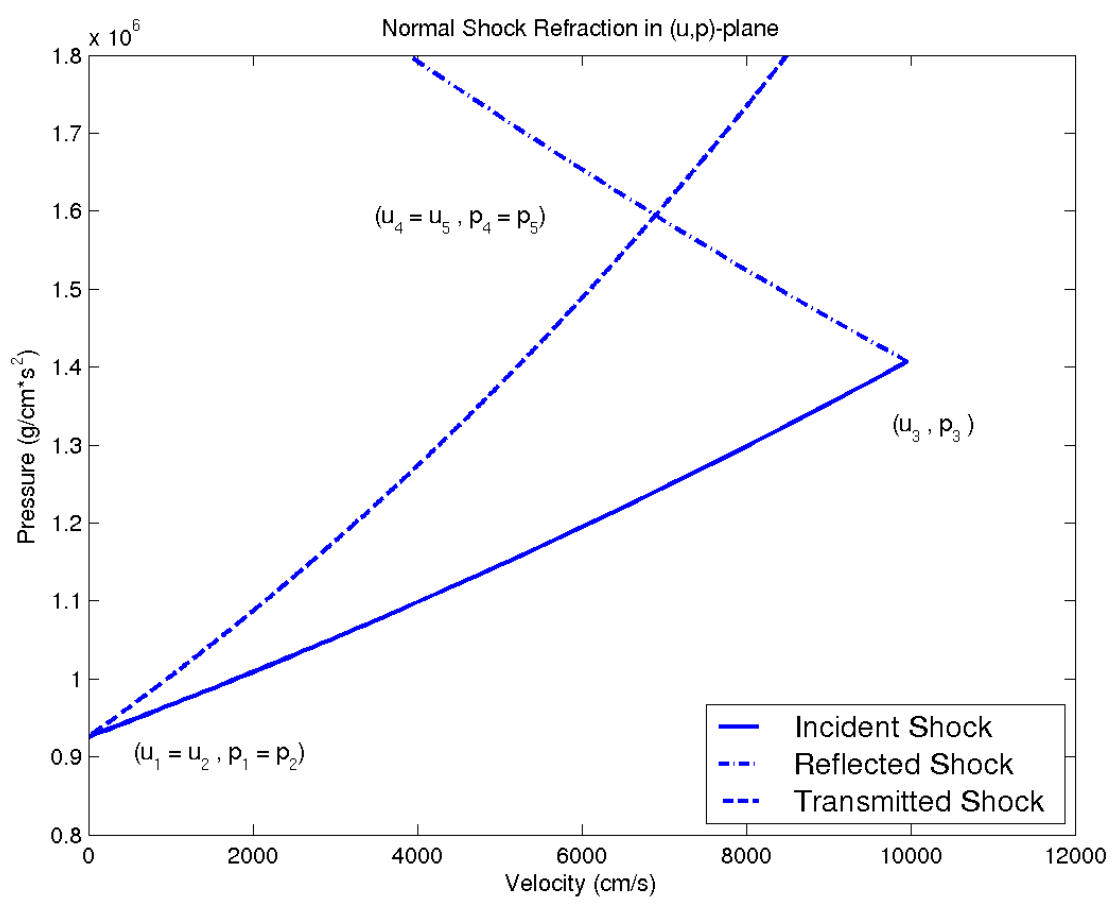

Figure 21: The normal shock refraction of a $M a=1.21$ shock wave in air(acetone) interacting with a contact surface separating air(acetone) and $\mathrm{SF}_{6}$ shown in the $(u, p)$-plane. The intersection of the curve representing the reflected shock with the curve representing the transmitted shock constitutes the solution of the refraction process.

\begin{tabular}{|c|c|c|}
\hline & $M a$ & $u_{\text {shock }}=c_{s} M a(\mathrm{~cm} / \mathrm{s})$ \\
\hline \hline Incident shock & 1.21 & 35774.5 \\
Reflected shock & 1.057855 & 32,756 \\
Transmitted shock & 1.30099 & 18,085 \\
\hline
\end{tabular}

Table 11: Analytical results for the shock Mach numbers and the shock velocities in the normal air(acetone)-SF 6 refraction. Note that the speed of the shock is reduced after refracting at the material interface. Therefore, this is a fast-slow refraction. 

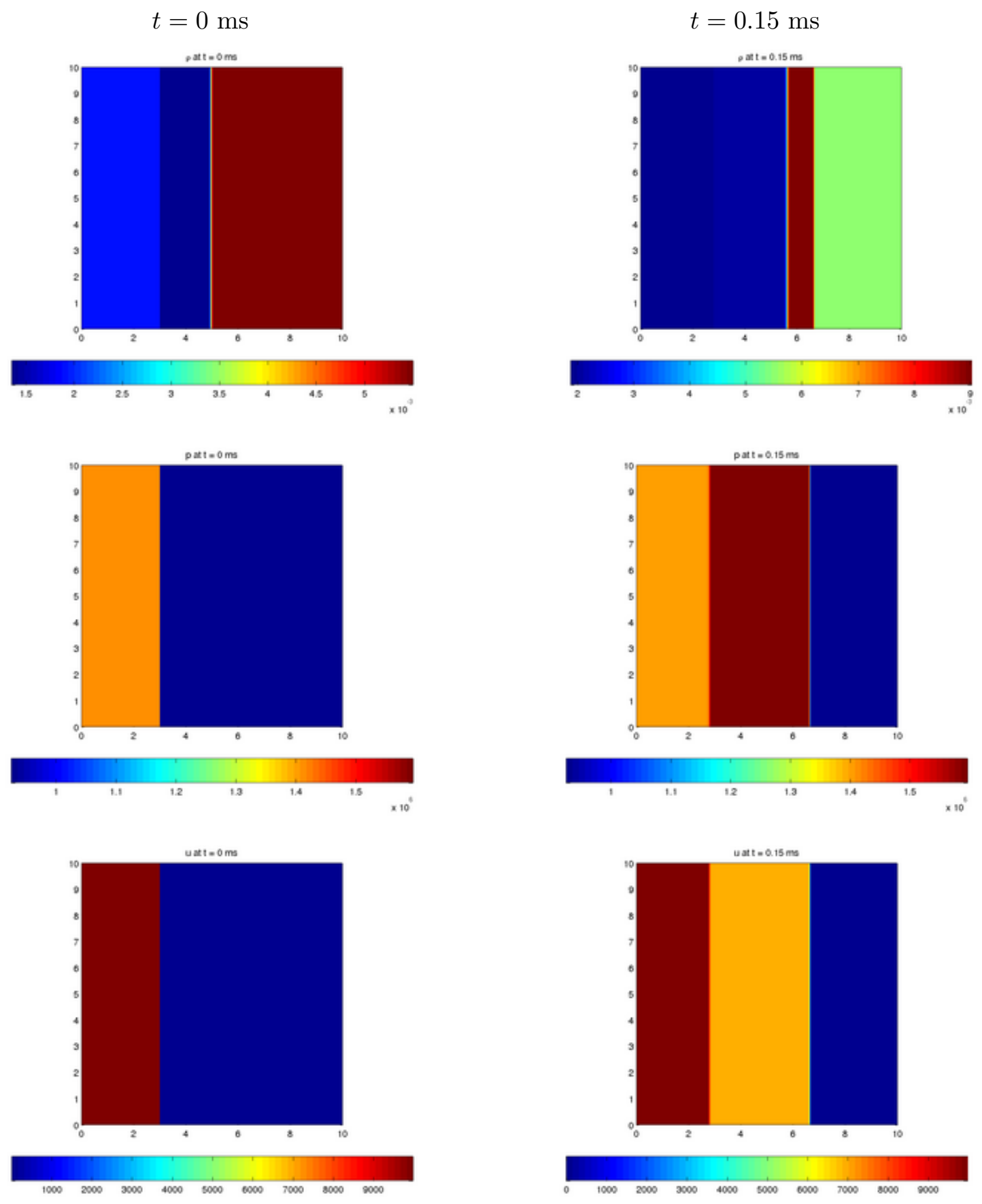

Figure 22: The density, pressure, and velocity $u$ at $t=0$ and $0.15 \mathrm{~ms}$ from a WENO simulation of a $M a=1.21$ shock in air(acetone) interacting with an air(acetone)-SF 6 interface. The figures at $t=0 \mathrm{~ms}$ show the incident shock, while the figures at $t=0.15 \mathrm{~ms}$ show the reflected and transmitted shock waves. 


\begin{tabular}{|c|c|c|c|}
\hline \multicolumn{4}{|c|}{ Region 1 } \\
\hline \hline & Analytical values & WENO results & Error $(\%)$ \\
\hline$\rho\left(\mathrm{g} / \mathrm{cm}^{3}\right)$ & $1.351 \times 10^{-3}$ & $1.351 \times 10^{-3}$ & 0 \\
$p\left(\mathrm{~g} / \mathrm{cm} \mathrm{s}^{2}\right)$ & $9.25511289 \times 10^{5}$ & $9.25511289 \times 10^{5}$ & 0 \\
$c_{s}(\mathrm{~cm} / \mathrm{s})$ & 29565.72 & 29565.72 & 0 \\
$T(\mathrm{~K})$ & 296 & 296 & 0 \\
$u(\mathrm{~cm} / \mathrm{s})$ & 0 & 0 & 0 \\
\hline \hline
\end{tabular}

\begin{tabular}{|c|c|c|c|}
\hline \hline \multicolumn{4}{|c|}{ Region 2 } \\
\hline \hline & Analytical values & WENO results & Error $(\%)$ \\
\hline$\rho\left(\mathrm{g} / \mathrm{cm}^{3}\right)$ & $5.494 \times 10^{-3}$ & $5.494 \times 10^{-3}$ & 0 \\
$p\left(\mathrm{~g} / \mathrm{cm} \mathrm{s}^{2}\right)$ & $9.25511289 \times 10^{5}$ & $9.25511289 \times 10^{5}$ & 0 \\
$c_{s}(\mathrm{~cm} / \mathrm{s})$ & 13569.27 & 13569.27 & 0 \\
$T(\mathrm{~K})$ & 295.91 & 295.91 & 0 \\
$u(\mathrm{~cm} / \mathrm{s})$ & 0 & 0 & 0 \\
\hline \hline
\end{tabular}

\begin{tabular}{|c|c|c|c|}
\hline \hline \multicolumn{4}{|c|}{ Region 3 } \\
\hline \hline$\rho\left(\mathrm{g} / \mathrm{cm}^{3}\right)$ & Analytical values & WENO results & Error $(\%)$ \\
$p\left(\mathrm{~g} / \mathrm{cm} \mathrm{s}^{2}\right)$ & $1.87261 \times 10^{-3}$ & $1.87260 \times 10^{-3}$ & 0.001 \\
$c_{s}(\mathrm{~cm} / \mathrm{s})$ & 30964.81 & 30964.80 & 0.001 \\
$T(\mathrm{~K})$ & 324.68 & 324.68 & 0.001 \\
$u(\mathrm{~cm} / \mathrm{s})$ & 9964.89 & 9964.97 & 0 \\
\hline \hline
\end{tabular}

\begin{tabular}{|c|c|c|c|}
\hline \hline \multicolumn{4}{|c|}{ Region 4 } \\
\hline \hline & Analytical values & WENO results & Error $(\%)$ \\
\hline$\rho\left(\mathrm{g} / \mathrm{cm}^{3}\right)$ & $9.0213 \times 10^{-3}$ & $9.0206 \times 10^{-3}$ & 0.08 \\
$p\left(\mathrm{~g} / \mathrm{cm} \mathrm{s}^{2}\right)$ & $1.594973 \times 10^{6}$ & $1.594872 \times 10^{6}$ & 0.001 \\
$c_{s}(\mathrm{~cm} / \mathrm{s})$ & 13901.15 & 13901.27 & 0.001 \\
$T(\mathrm{~K})$ & 310.56 & 310.57 & 0.003 \\
$u(\mathrm{~cm} / \mathrm{s})$ & 6902 & 6907.12 & 0.07 \\
\hline \hline
\end{tabular}

\begin{tabular}{|c|c|c|c|}
\hline \hline \multicolumn{4}{|c|}{ Region 5 } \\
\hline & Analytical values & WENO results & Error $(\%)$ \\
\hline \hline$\rho\left(\mathrm{g} / \mathrm{cm}^{3}\right)$ & $2.06573 \times 10^{-3}$ & $2.06571 \times 10^{-3}$ & 0.001 \\
$p\left(\mathrm{~g} / \mathrm{cm} \mathrm{s}^{2}\right)$ & $1.594973 \times 10^{6}$ & $1.594872 \times 10^{6}$ & 0.001 \\
$c_{s}(\mathrm{~cm} / \mathrm{s})$ & 31388.08 & 31387.27 & 0.003 \\
$T(\mathrm{~K})$ & 333.61 & 333.60 & 0.003 \\
$u(\mathrm{~cm} / \mathrm{s})$ & 6902 & 6907.12 & 0.07 \\
\hline \hline
\end{tabular}

Table 12: Results computed analytically and from the WENO simulation for a normal $M a=$ 1.21 shock refraction at an air(acetone)- $\mathrm{SF}_{6}$ interface in all of the five refractions regions. 


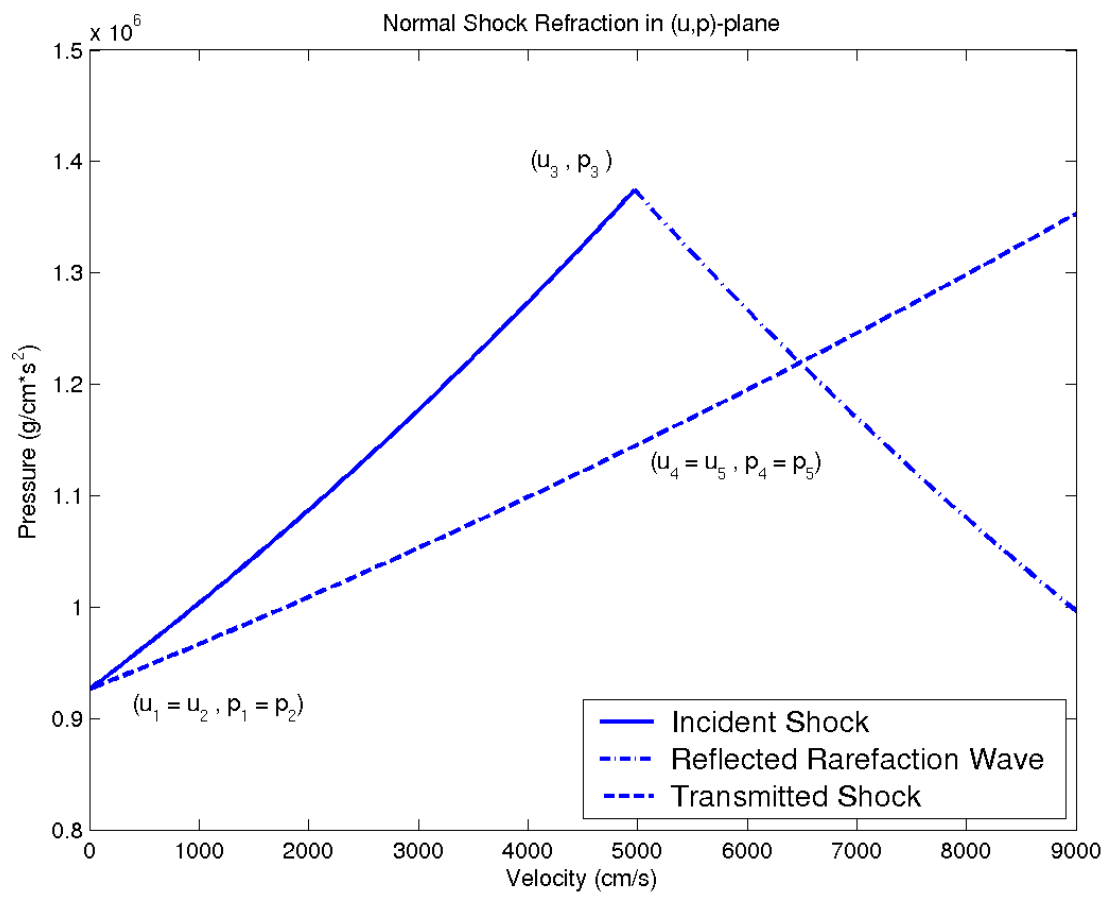

Figure 23: The refraction process for a $M a=1.2$ in $\mathrm{SF}_{6}$ interacting with a contact surface separating $\mathrm{SF}_{6}$ and air(acetone) shown in the $(u, p)$-plane. The intersection of the reflected rarefaction wave with the transmitted shock curves constitutes the solution of the refraction process.

\begin{tabular}{|c|c|c|}
\hline & $M a$ & $u_{\text {shock }}=c_{s} M a(\mathrm{~cm} / \mathrm{s})$ \\
\hline \hline Incident shock & 1.21 & 16421 \\
Transmitted shock & 1.132543 & 33415 \\
\hline
\end{tabular}

Table 13: Results for the shock Mach numbers and the shock velocities in the normal $\mathrm{SF}_{6}$ air(acetone) refraction. Note that the speed of the shock is increased following refraction, constituting a slow-fast refraction. 


$$
t=0 \mathrm{~ms}
$$
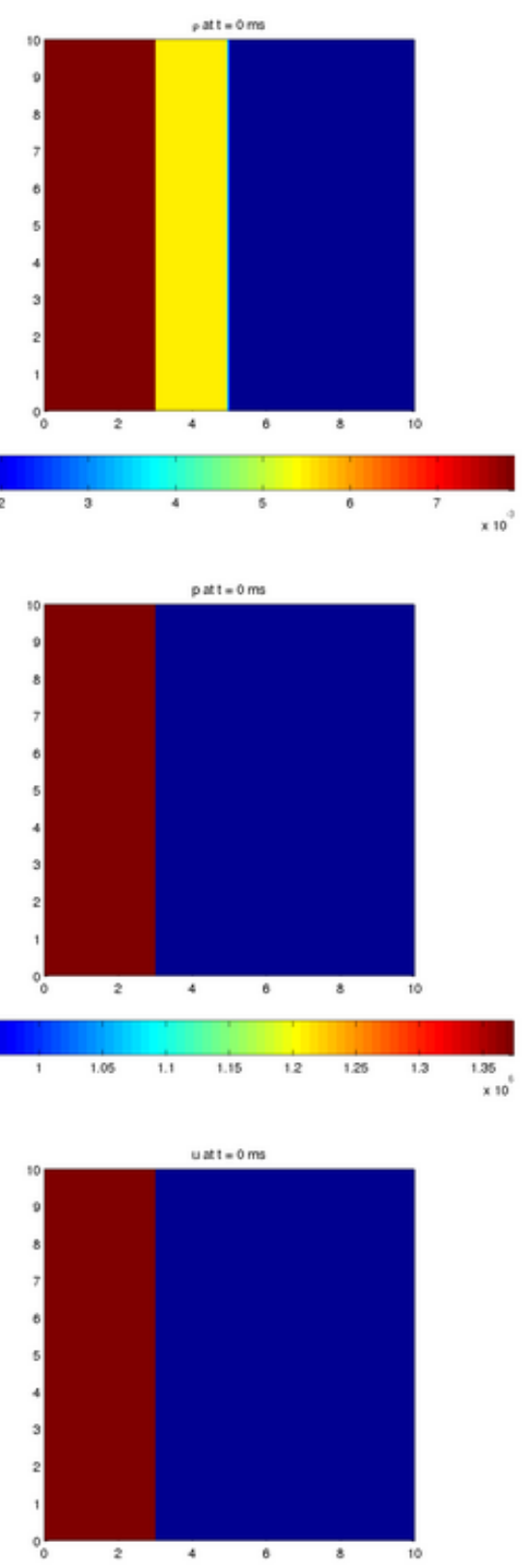

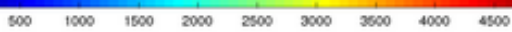

$$
t=0.15 \mathrm{~ms}
$$
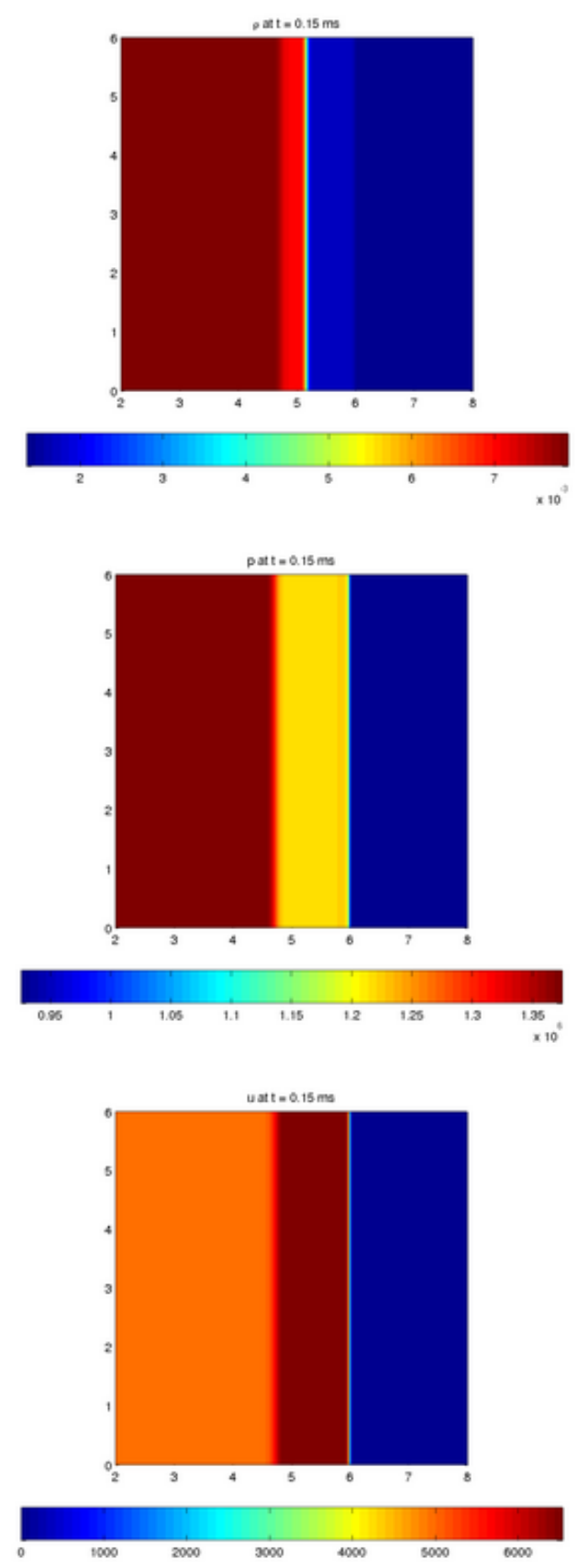

Figure 24: The density, pressure, and velocity $u$ at $t=0$ and $0.15 \mathrm{~ms}$ from a WENO simulation of the refraction observed when a normal $M a=1.21$ shock interacts with an air(acetone)-SF 6 interface. The figures at $t=0 \mathrm{~ms}$ show the incident shock, while the figures at $t=0.15 \mathrm{~ms}$ show the reflected rarefaction wave and the transmitted shock wave. 


\begin{tabular}{|c|c|c|c|}
\hline \multicolumn{4}{|c|}{ Region 1} \\
\hline & Analytical values & WENO results & Error $(\%)$ \\
\hline$\rho\left(\mathrm{g} / \mathrm{cm}^{3}\right)$ & $5.494 \times 10^{-3}$ & $5.494 \times 10^{-3}$ & 0 \\
\hline$p\left(\mathrm{~g} / \mathrm{cm} \mathrm{s}^{2}\right)$ & $9.257852 \times 10^{5}$ & $9.257852 \times 10^{5}$ & 0 \\
\hline$c_{s}(\mathrm{~cm} / \mathrm{s})$ & 13571.28 & 13571.28 & 0 \\
\hline$T(\mathrm{~K})$ & 296 & 296 & 0 \\
\hline$u(\mathrm{~cm} / \mathrm{s})$ & 0 & 0 & 0 \\
\hline \multicolumn{4}{|c|}{ Region 2} \\
\hline & " Analytical values & "WENO results & Error (\%) \\
\hline$\rho\left(\mathrm{g} / \mathrm{cm}^{3}\right)$ & $1.351 \times 10^{-3}$ & $1.351 \times 10^{-3}$ & 0 \\
\hline$p\left(\mathrm{~g} / \mathrm{cm} \mathrm{s}^{2}\right)$ & $9.257852 \times 10^{5}$ & $9.257852 \times 10^{5}$ & 0 \\
\hline$c_{s}(\mathrm{~cm} / \mathrm{s})$ & 29570.1 & 29570.1 & 0 \\
\hline$T(\mathrm{~K})$ & 296.09 & 296.09 & 0 \\
\hline$u(\mathrm{~cm} / \mathrm{s})$ & 0 & 0 & 0 \\
\hline \multicolumn{4}{|c|}{ Region 3} \\
\hline & "Analytical values & WENO results & Error (\%) \\
\hline$\rho\left(\mathrm{g} / \mathrm{cm}^{3}\right)$ & $7.8812 \times 10^{-3}$ & $7.8804 \times 10^{-3}$ & 0.01 \\
\hline$p\left(\mathrm{~g} / \mathrm{cm} \mathrm{s}^{2}\right)$ & $1.3745 \times 10^{6}$ & $1.3744 \times 10^{6}$ & 0.007 \\
\hline$c_{s}(\mathrm{~cm} / \mathrm{s})$ & 13806.77 & 13806.65 & 0.001 \\
\hline$T(\mathrm{~K})$ & 306.36 & 306.37 & 0.001 \\
\hline$u(\mathrm{~cm} / \mathrm{s})$ & 4974 & 4975.5 & 0.03 \\
\hline \multicolumn{4}{|c|}{ Region 4} \\
\hline & Analytical values & WENO results & Error (\%) \\
\hline$\rho\left(\mathrm{g} / \mathrm{cm}^{3}\right)$ & $1.6754 \times 10^{-3}$ & $1.6789 \times 10^{-3}$ & 0.2 \\
\hline$p\left(\mathrm{~g} / \mathrm{cm} \mathrm{s}^{2}\right)$ & $1.219195 \times 10^{6}$ & $1.219105 \times 10^{6}$ & 0.001 \\
\hline$c_{s}(\mathrm{~cm} / \mathrm{s})$ & 30471 & 30439 & 0.05 \\
\hline$T(\mathrm{~K})$ & 314.4 & 313.7 & 0.22 \\
\hline$u(\mathrm{~cm} / \mathrm{s})$ & 6485 & 6489.5 & 0.07 \\
\hline \multicolumn{4}{|c|}{ Region 5} \\
\hline & Analytical values & WENO results & Error (\%) \\
\hline$\rho\left(\mathrm{g} / \mathrm{cm}^{3}\right)$ & $7.0622 \times 10^{-3}$ & $7.0595 \times 10^{-3}$ & 0.08 \\
\hline$p\left(\mathrm{~g} / \mathrm{cm} \mathrm{s}^{2}\right)$ & $1.219195 \times 10^{6}$ & $1.219105 \times 10^{6}$ & 0.001 \\
\hline$c_{s}(\mathrm{~cm} / \mathrm{s})$ & 13736.4 & 13738.9 & 0.01 \\
\hline$T(\mathrm{~K})$ & 303.25 & 303.35 & 0.001 \\
\hline$u(\mathrm{~cm} / \mathrm{s})$ & 6485 & 6489.5 & 0.07 \\
\hline
\end{tabular}

Table 14: Results computed analytically and from a WENO simulation for a normal $M a=1.21$ shock refraction at an $\mathrm{SF}_{6}$-air-acetone interface when a reflected rarefaction wave is observed. Results in all of the five refractions regions are shown. 


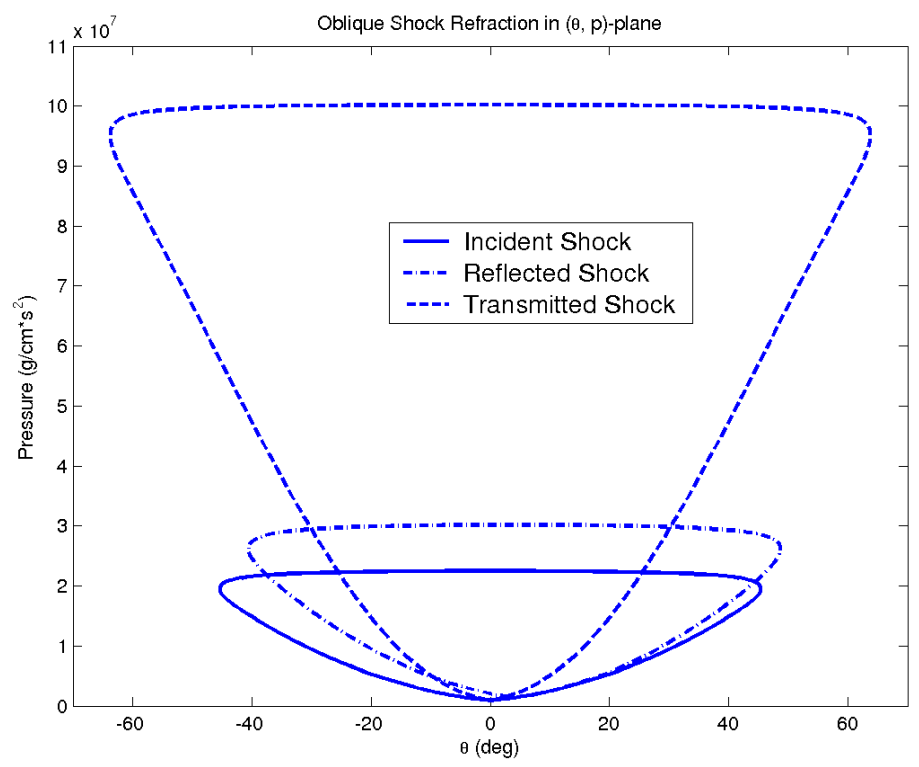

Figure 25: Shock polars for the incident, reflected, and transmitted shocks in air(acetone)-SF 6 oblique shock refraction in the $(\theta, p)$-plane. Note that the transmitted shock polar originates right on top of the incident shock polar, but shifted by the amount $\theta_{i}$ and $p_{3}$ corresponding to the state behind the incident shock. The points where the reflected shock polar and the transmitted shock polar intersect correspond to the solution of the refraction problem. At those points the pressure $p_{5}=p_{4}$ is matched and the flow turning angles satisfy $\theta_{t}=\theta_{i}+\theta_{r}$.

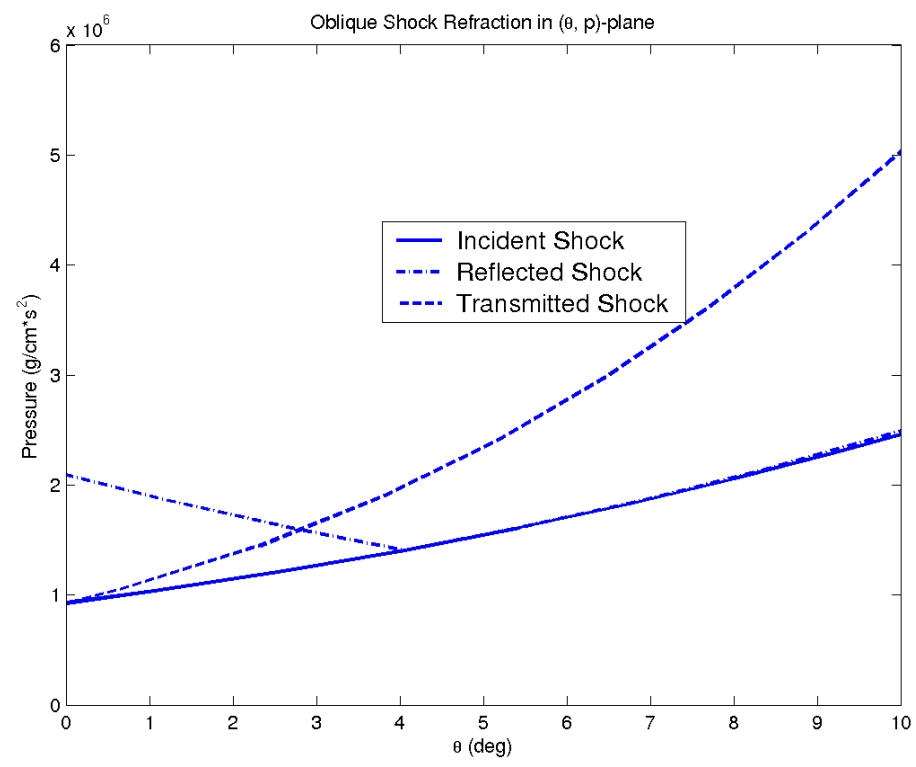

Figure 26: A close up of Fig. 25 for the solution with the smallest change in pressure. 


\begin{tabular}{|c|c|c|c|}
\hline & $\mathrm{Ma}$ & $\beta\left(^{\circ}\right)$ & $\theta\left(^{\circ}\right)$ \\
\hline \hline Incident shock & 4.675 & 15 & 4.059 \\
Reflected shock & 4.3916 & 166 & -1.261 \\
Transmitted shock & 9.8917 & 7.34 & 2.857 \\
\hline
\end{tabular}

Table 15: Analytical results for the shock Mach numbers, shock-interface angle $\beta$, and flow turning angle $\theta$ for the oblique air(acetone)-SF 6 refraction.

\subsubsection{Oblique shock refraction at an $\mathrm{SF}_{6}$-air(acetone) interface with a reflected rarefaction wave}

Finally, consider a contact surface separating $\mathrm{SF}_{6}$ and air(acetone) forming an angle $\beta_{\text {inter face }}=$ $75^{\circ}$ with the plane of propagation of the shock. Note that the shock and the interface form an angle $\beta_{i}=15^{\circ}$. The shock polar is presented in Fig. 28 with a close up in Fig. 29 . The analytical results for the shock Mach number, shock interface angle $\beta$, and flow turning angle $\theta$ are presented in Table 17. Results for the flow ahead of and behind the reflected rarefaction wave are presented in Table 19 . Images from the WENO simulations are shown in Fig. 30. Presented are contour plots for the density, pressure, and horizontal and vertical velocity at $t=0.1 \mathrm{~ms}$. The analytical and numerical results are compared in Table 19 and are in excellent agreement. 


\begin{tabular}{|c|c|c|c|}
\hline \multicolumn{4}{|c|}{ Region 1 } \\
\hline \hline & Analytical values & WENO results & Error $(\%)$ \\
\hline$\rho\left(\mathrm{g} / \mathrm{cm}^{3}\right)$ & $1.351 \times 10^{-3}$ & $1.351 \times 10^{-3}$ & 0 \\
$p\left(\mathrm{~g} / \mathrm{cm} \mathrm{s}^{2}\right)$ & $9.25511289 \times 10^{5}$ & $9.25511289 \times 10^{5}$ & 0 \\
$c_{s}(\mathrm{~cm} / \mathrm{s})$ & 29565.72 & 29565.72 & 0 \\
$T(\mathrm{~K})$ & 296 & 296 & 0 \\
$u(\mathrm{~cm} / \mathrm{s})$ & & 0 & \\
$v(\mathrm{~cm} / \mathrm{s})$ & & 0 & \\
\hline \hline
\end{tabular}

\begin{tabular}{|c|c|c|c|}
\hline \hline \multicolumn{4}{|c|}{ Region 2 } \\
\hline \hline$\rho\left(\mathrm{g} / \mathrm{cm}^{3}\right)$ & Analytical values & WENO results & Error $(\%)$ \\
$p\left(\mathrm{~g} / \mathrm{cm} \mathrm{s}^{2}\right)$ & $9.494 \times 10^{-3}$ & $5.494 \times 10^{-3}$ & 0 \\
$c_{s}(\mathrm{~cm} / \mathrm{s})$ & 13569.27 & 13569.27 & 0 \\
$T(\mathrm{~K})$ & 295.91 & 295.91 & 0 \\
$u(\mathrm{~cm} / \mathrm{s})$ & & 0 & 0 \\
$v(\mathrm{~cm} / \mathrm{s})$ & & 0 & \\
\hline \hline
\end{tabular}

\begin{tabular}{|c|c|c|c|}
\hline \hline \multicolumn{4}{|c|}{ Region 3 } \\
\hline \hline$\rho\left(\mathrm{g} / \mathrm{cm}^{3}\right)$ & Analytical values & WENO results & Error $(\%)$ \\
$p\left(\mathrm{~g} / \mathrm{cm} \mathrm{s}^{2}\right)$ & $1.47261 \times 10^{-3}$ & $1.87260 \times 10^{-3}$ & 0.001 \\
$c_{s}(\mathrm{~cm} / \mathrm{s})$ & 30964.81 & $1.407123 \times 10^{6}$ & 0.001 \\
$T(\mathrm{~K})$ & 324.68 & 30964.80 & 0.001 \\
$u(\mathrm{~cm} / \mathrm{s})$ & & 324.68 & 0 \\
$v(\mathrm{~cm} / \mathrm{s})$ & & 9964.61 & \\
\hline \hline
\end{tabular}

\begin{tabular}{|c|c|c|c|}
\hline \hline \multicolumn{4}{|c|}{ Region 4 } \\
\hline \hline & Analytical values & WENO results & Error $(\%)$ \\
\hline$\rho\left(\mathrm{g} / \mathrm{cm}^{3}\right)$ & $9.02646 \times 10^{-3}$ & $8.99 \times 10^{-3}$ & 0.34 \\
$p\left(\mathrm{~g} / \mathrm{cm} \mathrm{s}^{2}\right)$ & $1.5959 \times 10^{6}$ & $1.5889 \times 10^{6}$ & 0.44 \\
$c_{s}(\mathrm{~cm} / \mathrm{s})$ & 13901.6 & 13898.9 & 0.02 \\
$T(\mathrm{~K})$ & 310.6 & 310.5 & 0.03 \\
$u(\mathrm{~cm} / \mathrm{s})$ & & 6793.9 & \\
$v(\mathrm{~cm} / \mathrm{s})$ & & -900 & \\
\hline \hline \hline
\end{tabular}

\begin{tabular}{|c|c|c|c|}
\hline \hline \multicolumn{4}{|c|}{ Region 5 } \\
\hline \hline$\rho\left(\mathrm{g} / \mathrm{cm}^{3}\right)$ & Analytical values & WENO results & Error $(\%)$ \\
$p\left(\mathrm{~g} / \mathrm{cm} \mathrm{s}^{2}\right)$ & $2.0666 \times 10^{-3}$ & $2.0593 \times 10^{-3}$ & 0.35 \\
$c_{s}(\mathrm{~cm} / \mathrm{s})$ & 31385.8 & $1.5889 \times 10^{6}$ & 0.44 \\
$T(\mathrm{~K})$ & 333.6 & 31377 & 0.03 \\
$u(\mathrm{~cm} / \mathrm{s})$ & & 333.4 & 0.06 \\
$v(\mathrm{~cm} / \mathrm{s})$ & & 7275 & \\
\hline \hline
\end{tabular}

Table 16: Results computed analytically and from WENO simulation for an oblique $M a=1.21$ shock refraction at a air(acetone)-SF $\mathrm{SF}_{6}$ interface when the interface angle is $\beta_{\text {interface }}=75^{\circ}$. Results are shown in all of the five refractions regions. 

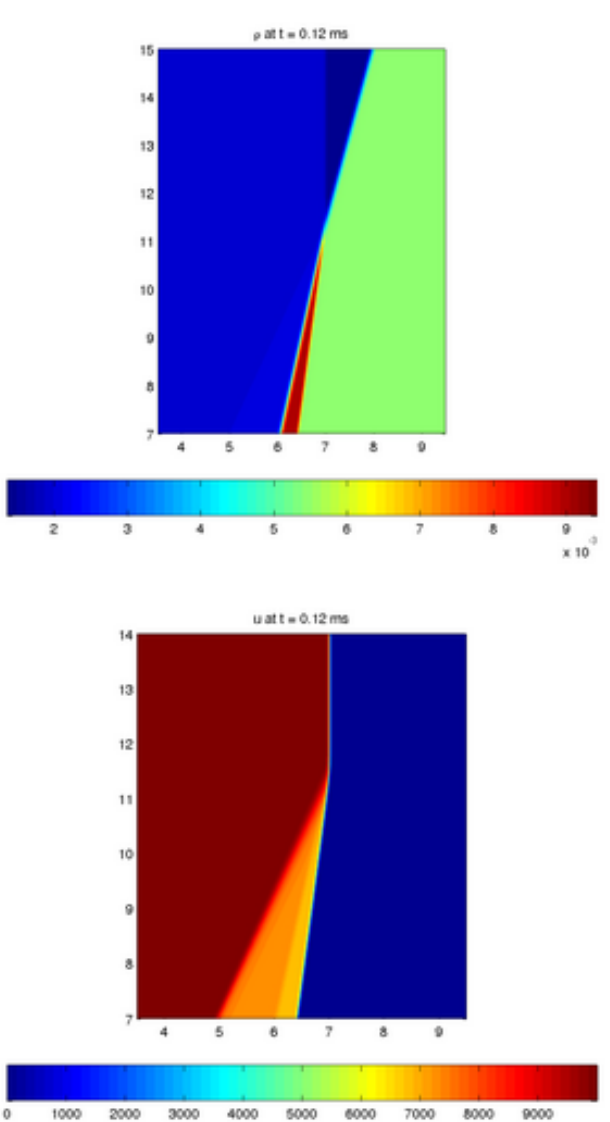
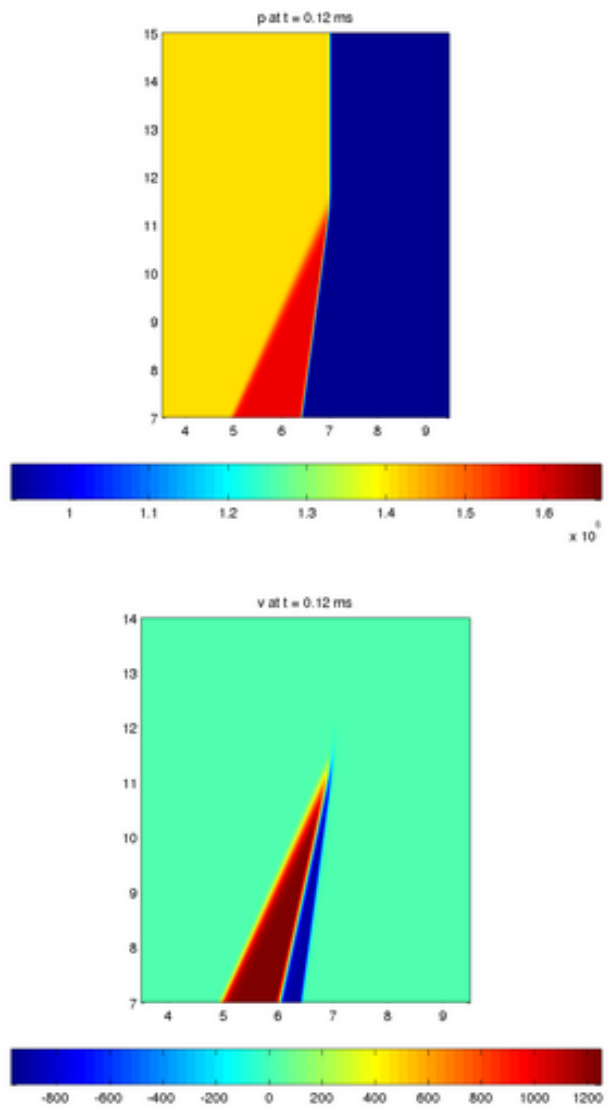

Figure 27: The density, pressure, and horizontal and vertical velocity from a WENO simulation observed when a $M a=1.21$ shock in air(acetone) refracts at an $\mathrm{SF}_{6}$ interface. The angle between the interface and the plane of motion of the shock is $\beta=75^{\circ}$. The density, pressure, horizontal velocity $u$, and vertical velocity $v$ are shown at $t=0.012 \mathrm{~ms}$. All of the waves coincide at the triple-point; therefore, each plot presents a view of all five refraction regions.

\begin{tabular}{|c|c|c|c|}
\hline & $M a$ & $\beta\left(^{\circ}\right)$ & $\theta\left(^{\circ}\right)$ \\
\hline \hline Incident shock & 4.6751 & 15 & 4.42 \\
Reflected rarefaction wave & & & 1.158 \\
Transmitted shock & 1.967348 & 32.14 & 5.579 \\
\hline
\end{tabular}

Table 17: Analytical results for the shock Mach numbers, shock-interface angles $\beta$, and flow turning angles $\theta$ for the system of waves observed during the oblique $\mathrm{SF}_{6}$-air(acetone) refraction. 


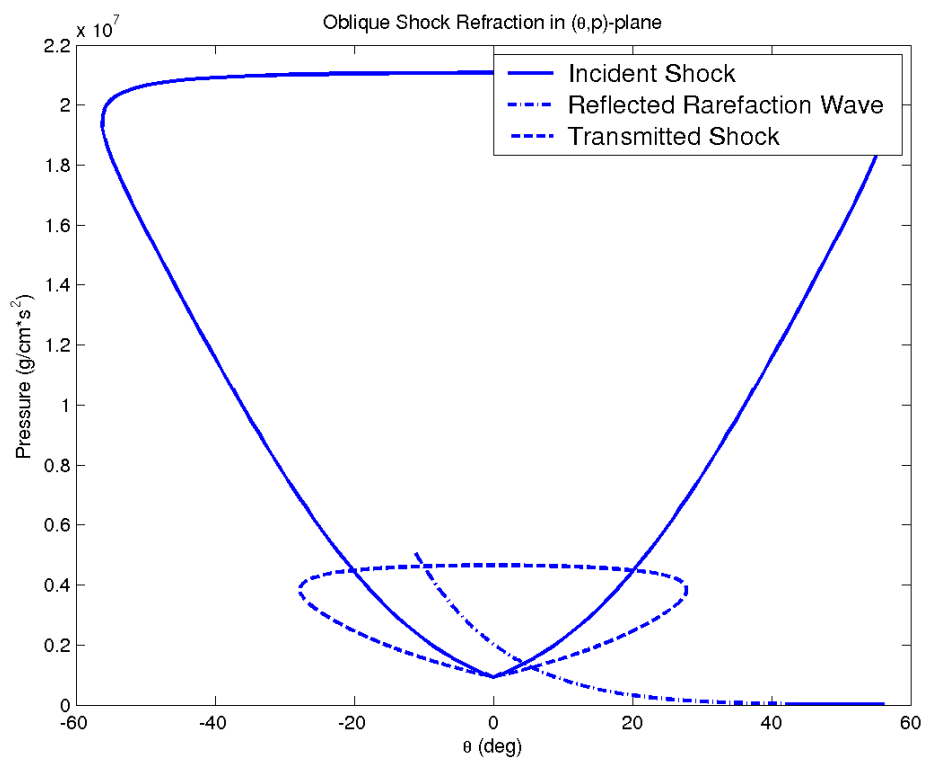

Figure 28: Shock polars for the incident shock, reflected rarefaction wave, and the transmitted shock for the $\mathrm{SF}_{6}$-air(acetone) oblique shock refraction process in the $(\theta, p)$-plane. The reflected rarefaction wave polar intersects the incident shock polar at the location $p_{3}, \theta_{i}$. The points where the polar for the reflected rarefaction wave and the transmitted shock polar intersect correspond to solution of the refraction problem. At those points, the pressure $p_{5}=p_{4}$ is matched and the flow turning angles satisfy $\theta_{t}=\theta_{i}+\theta_{r}$.

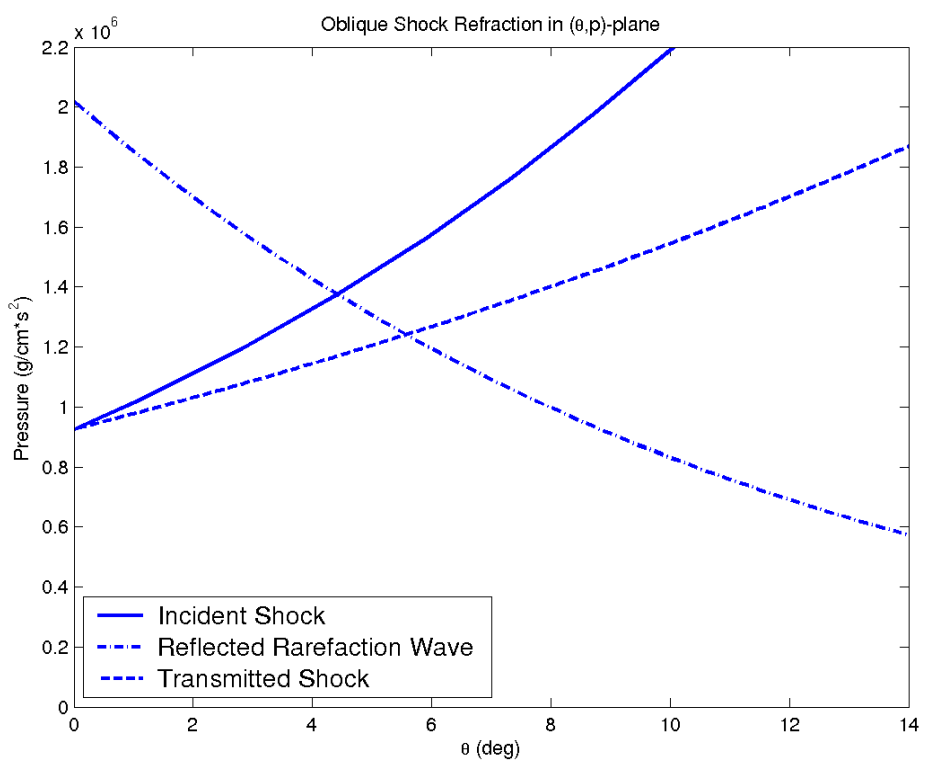

Figure 29: A close up of Fig. 28. The intersection of the polar for the reflected rarefaction wave with the transmitted shock polar constitutes the solution of the refraction process. 


\begin{tabular}{|c|c|c|}
\hline & $M a$ & $\Theta(M a)\left(^{\circ}\right)$ \\
\hline \hline Region 3 & 4.5155 & 126.16 \\
Region 5 & 4.5561 & 127.32 \\
\hline
\end{tabular}

Table 18: The flow Mach numbers ahead of and behind the reflected rarefaction wave, and the value of the Prandtl-Meyer angles determined analytically for the oblique $\mathrm{SF}_{6}$-air(acetone) refraction. Note that the flow is accelerated across a rarefaction wave, which is evident from the increase in Mach number.
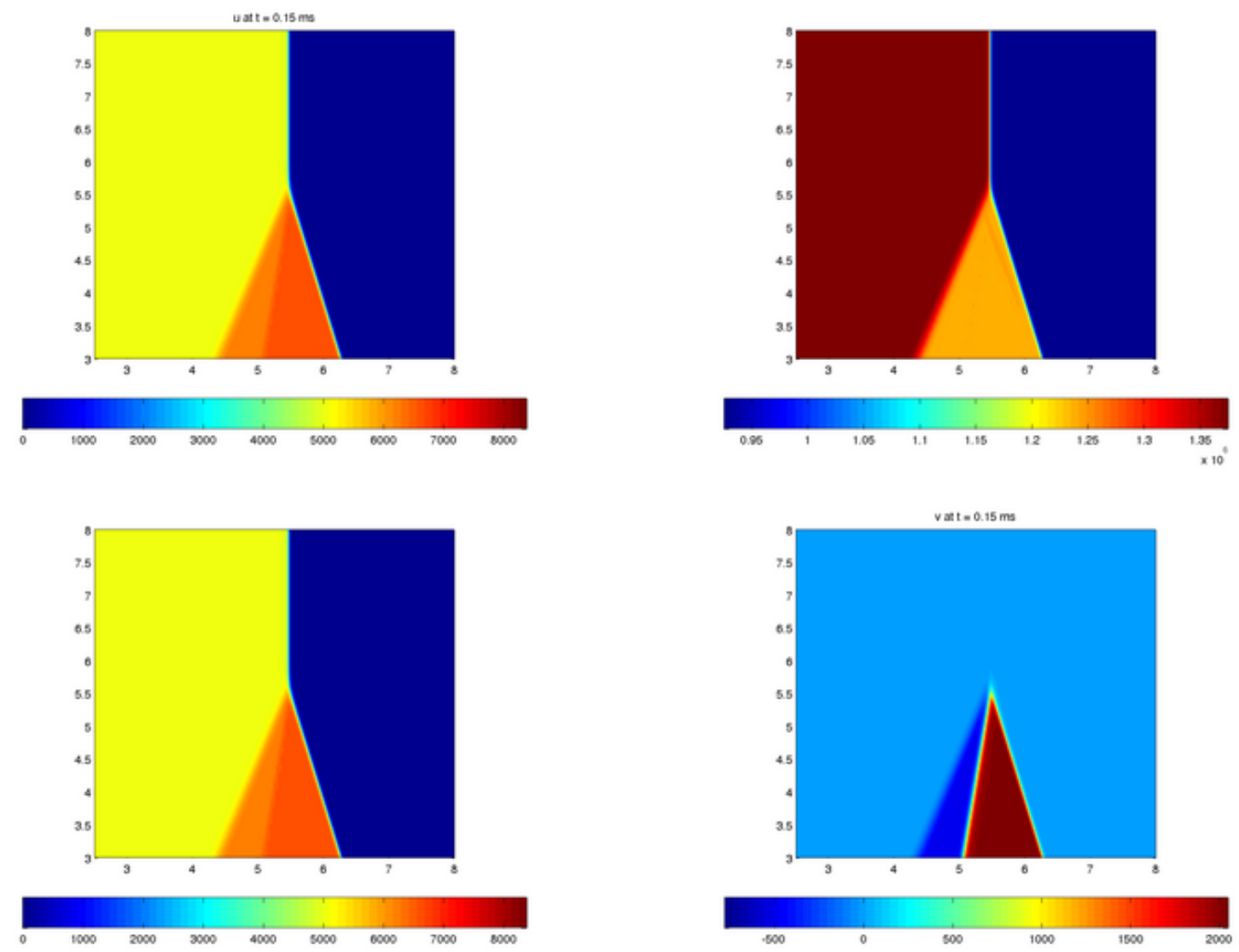

Figure 30: The density, pressure, horizontal velocity $u$, and vertical velocity $v$ at $t=0.01 \mathrm{~ms}$ from a WENO simulation of a $M a=1.21$ shock in $\mathrm{SF}_{6}$ refracting into air(acetone) when the interface is at angle of $\beta_{\text {interface }}=75^{\circ}$ relative to the plane of motion of the shock. All of the waves coincide at the triple-point. 


\begin{tabular}{|c|c|c|c|}
\hline \multicolumn{4}{|c|}{ Region 1 } \\
\hline \hline & Analytical values & WENO results & Error $(\%)$ \\
\hline$\rho\left(\mathrm{g} / \mathrm{cm}^{3}\right)$ & $5.494 \times 10^{-3}$ & $5.494 \times 10^{-3}$ & 0 \\
$p\left(\mathrm{~g} / \mathrm{cm} \mathrm{s}^{2}\right)$ & $9.257852 \times 10^{5}$ & $9.257852 \times 10^{5}$ & 0 \\
$c_{s}(\mathrm{~cm} / \mathrm{s})$ & 13571.28 & 13571.28 & 0 \\
$T(\mathrm{~K})$ & 296 & 296 & 0 \\
$u(\mathrm{~cm} / \mathrm{s})$ & & 0 & \\
$v(\mathrm{~cm} / \mathrm{s})$ & & 0 & \\
\hline \hline
\end{tabular}

\begin{tabular}{|c|c|c|c|}
\hline \hline \multicolumn{4}{|c|}{ Region 2 } \\
\hline \hline & Analytical values & WENO results & Error $(\%)$ \\
\hline$\rho\left(\mathrm{g} / \mathrm{cm}^{3}\right)$ & $1.351 \times 10^{-3}$ & $1.351 \times 10^{-3}$ & 0 \\
$p\left(\mathrm{~g} / \mathrm{cm} \mathrm{s}^{2}\right)$ & $9.257852 \times 10^{5}$ & $9.257852 \times 10^{5}$ & 0 \\
$c_{s}(\mathrm{~cm} / \mathrm{s})$ & 29570.1 & 29570.1 & 0 \\
$T(\mathrm{~K})$ & 296.09 & 296.09 & 0 \\
$u(\mathrm{~cm} / \mathrm{s})$ & & 0 & \\
$v(\mathrm{~cm} / \mathrm{s})$ & & 0 & \\
\hline \hline
\end{tabular}

\begin{tabular}{|c|c|c|c|}
\hline \hline \multicolumn{4}{|c|}{ Region 3 } \\
\hline \hline & Analytical values & WENO results & Error $(\%)$ \\
\hline$\rho\left(\mathrm{g} / \mathrm{cm}^{3}\right)$ & $7.8812 \times 10^{-3}$ & $7.8804 \times 10^{-3}$ & 0.01 \\
$p\left(\mathrm{~g} / \mathrm{cm} \mathrm{s}^{2}\right)$ & $1.3745 \times 10^{6}$ & $1.3744 \times 10^{6}$ & 0.007 \\
$c_{s}(\mathrm{~cm} / \mathrm{s})$ & 13806.77 & 13806.65 & 0.001 \\
$T(\mathrm{~K})$ & 306.36 & 306.37 & 0.001 \\
$u(\mathrm{~cm} / \mathrm{s})$ & & 4975.5 & \\
$v(\mathrm{~cm} / \mathrm{s})$ & & 0 & \\
\hline \hline
\end{tabular}

\begin{tabular}{|c|c|c|c|}
\hline \hline \multicolumn{4}{|c|}{ Region 4 } \\
\hline \hline & Analytical values & WENO results & Error $(\%)$ \\
\hline$\rho\left(\mathrm{g} / \mathrm{cm}^{3}\right)$ & $1.6979 \times 10^{-3}$ & $1.6974 \times 10^{-3}$ & 0.03 \\
$p\left(\mathrm{~g} / \mathrm{cm} \mathrm{s}^{2}\right)$ & $1.24024 \times 10^{6}$ & $1.24001 \times 10^{6}$ & 0.008 \\
$c_{s}(\mathrm{~cm} / \mathrm{s})$ & 30529.8 & 30529.7 & 0.001 \\
$T(\mathrm{~K})$ & 315.6 & 315.6 & 0 \\
$u(\mathrm{~cm} / \mathrm{s})$ & & 6586.08 & \\
$v(\mathrm{~cm} / \mathrm{s})$ & & 2030 & \\
\hline \hline
\end{tabular}

\begin{tabular}{|c|c|c|c|}
\hline \hline \multicolumn{4}{|c|}{ Region 5 } \\
\hline \hline & Analytical values & WENO results & Error $(\%)$ \\
\hline$\rho\left(\mathrm{g} / \mathrm{cm}^{3}\right)$ & $7.1741 \times 10^{-3}$ & $7.172 \times 10^{-3}$ & 0.03 \\
$p\left(\mathrm{~g} / \mathrm{cm} \mathrm{s}^{2}\right)$ & $1.24024 \times 10^{6}$ & $1.24001 \times 10^{6}$ & 0.008 \\
$c_{s}(\mathrm{~cm} / \mathrm{s})$ & 13746.5 & 13746.4 & 0.01 \\
$T(\mathrm{~K})$ & 303.69 & 303.68 & 0.001 \\
$u(\mathrm{~cm} / \mathrm{s})$ & & 6172 & \\
$v(\mathrm{~cm} / \mathrm{s})$ & & -500 & \\
\hline \hline
\end{tabular}

Table 19: Results computed analytically and from a WENO simulation for an oblique $M a=$ 1.21 shock refraction at an $\mathrm{SF}_{6}$-air(acetone) interface when the interface angle is $\beta=75^{\circ}$. A reflected rarefaction wave is observed. Results from all of the five refraction regions are shown. 


\section{Conclusions}

In the present work, shock refraction was chosen as an example of a two-fluid, complex hydrodynamic flow suitable for code validation. Shock refraction can be considered as a building block for the investigation of more complex shock-induced hydrodynamic instabilities such as the Richtmyer-Meshkov instability [24]. Furthermore, exact analytical results can be obtained for regular shock refraction using shock polar analysis and these results can be compared to those obtained from numerical simulations. Shock refraction is also an example of a complex flow for which experimental data is available for comparison to numerical simulation results [19].

A validation of the weighted essentially non-oscillatory (WENO) shock-capturing method (implemented in the HOPE code) based on shock refraction was performed as follows. Normal and oblique regular shock refraction were investigated when reflected shock waves (light-toheavy) and reflected rarefaction waves (heavy-to-light) are observed. Two sets of gases were considered: argon/xenon with the same adiabatic exponent $\gamma$ to validate the single-fluid implementation, and air(acetone) $/ \mathrm{SF}_{6}$ with different adiabatic exponents to validate the two-fluid ('gamma-blending') implementation. The method/code validation consisted of two steps:

1. for each of the eight cases considered, analytical methods based on shock polar analysis were applied to determine the state of the gases in each of the five regions observed in regular shock refraction, and;

2. numerical simulations were performed and values from the five refraction regions were compared with the analytical predictions.

In all cases, excellent agreement was observed between the numerical simulation results and the analytical results. To our knowledge, shock refraction has not been previously used as a validation of the WENO method. Subsequent reports will detail two- and three-dimensional simulations and analysis of other complex hydrodynamic flows using the HOPE code including the single-mode Richtmyer-Meshkov instability with reshock (Part 2 [24]), the oblique single-mode Richtmyer-Meshkov instability (Part 3 [25]), and multi-mode Richtmyer-Meshkov instability (Part 4 [26]).

\section{Acknowledgments}

The authors thank Dr. Wai-Sun Don of Brown University for assistance with all aspects of the HOPE code. Marco Latini also acknowledges support from the Air Force Office of Scientific Research through the National Defense Science and Engineering Graduate Fellowship. This work was performed under the auspices of the U.S. Department of Energy by the University of California, Lawrence Livermore National Laboratory under Contract No. W-7405-Eng-48. 


\section{References}

[1] A. M. Abd-El-Fattah and L. F. Henderson, Shock waves at a fast-slow gas interface, J. Fluid Mech. 86 (1978), 15-32.

[2] A. M. Abd-El-Fattah, L. F. Henderson, and A. Lozzi, Precursor shock waves at a slow-fast gas interface, J. Fluid Mech. 76 (1976), 157-176.

[3] R. Abgrall and S. Karni, Computations of compressible multifluids, J. Comput. Phys 169 (2001), 594-623.

[4] D. Balsara and C.-W Shu, Monotonicity preserving weighted essentially non-oscillatory schemes with increasingly high order of accuracy, J. Comput. Phys. 160 (2000), 405-452.

[5] G. Ben-Dor, I. Ozer, and T. Elperin (eds.), Handbook of Shock Waves. Vol. I, Academic Press, San Diego, 2001.

[6] G. Ben-Dor, I. Ozer, and T. Elperin (eds.), Handbook of Shock Waves. Vol. II, Academic Press, San Diego, 2001.

[7] P. Colella and P. R. Woodward, The piecewise parabolic method (PPM) for gas-dynamical simulations, J. Comput. Phys. 54 (1984), 174-201.

[8] B. D. Collins and J. W. Jacobs, PLIF flow visualization and measurements of the Richtmyer-Meshkov instability of an air/SF6 interface, J. Fluid Mech. 464 (2002), 113136.

[9] R. Courant and K. O. Friedrichs, Supersonic Flow and Shock Waves, Springer-Verlag, New York, 1992.

[10] W. S. Don, D. Gottlieb, and C.-W. Shu, High order accurate simulations and physics of shock-induced turbulent mixing, Contractor Report, Brown University (2004).

[11] R. P. Fedkiw, B. Merriman, and S. Osher, Efficient characteristic projection in upwind difference schemes for hyperbolic systems, J. Comput. Phys. 141 (1998), 22-36.

[12] Jahn R. G., The refraction of shock waves at a gaseous interface, J. Fluid Mech. 1 (1956), 457-489.

[13] A. Harten, High resolution schemes for hyperbolic conservation laws, J. Comput. Phys. 49 (1983), 357-393.

[14] A. Harten, B. Engquist, S. Osher, and S. R. Chakravarthy, Uniformly high order accurate essentially non-oscillatory schemes, III, J. Comput. Phys. 77 (1987), 231-303.

[15] L. F. Henderson, The refraction of shock waves at a gaseous interface, J. Fluid Mech. 1 (1956), 457-489.

[16] The refraction of a plane shock wave at a gas interface, J. Fluid Mech. 26 (1966), $607-637$.

[17]_ On the refraction of shock waves, J. Fluid Mech. 198 (1989), 365-386. 
[18] L. F. Henderson and Lozzi A., Experiments on transition of mach reflexion, J. Fluid Mech. 68 (1975), 139-155.

[19] L. F. Henderson, Colella P., and Puckett E. G., The refraction of shock waves at a slow-fast gas interface, J. Fluid Mech. 224 (1991), 1-27.

[20] D. J. Hill and D. I. Pullin, Hybrid tuned center-difference-WENO method for large eddy simulations in the presence of strong shocks, J. Comput. Phys. 194 (2004), 435-450.

[21] G.-S. Jiang and C.-W Shu, Efficient implementation of weighted ENO schemes, J. Comput. Phys. 126 (1996), 202-228.

[22] S. Karni, Hybrid multifluid algorithms, SIAM J. Sci. Comput. 17 (1996), 1019-1039.

[23] C. B. Laney, Computational Gasdynamics, Cambridge University Press, New York, 1998.

[24] M. Latini and O. Schilling, Weighted Essentially Non-Oscillatory Simulations and Modeling of Complex Hydrodynamic Flows. Part 2. Single-Mode Richtmyer-Meshkov Instability with Reshock, Tech. report, Lawrence Livermore National Laboratory, 2004.

[25] _ Weighted Essentially Non-Oscillatory Simulations and Modeling of Complex Hydrodynamic Flows. Part 3. The Oblique Shock Richtmyer-Meshkov Instability, Tech. report, Lawrence Livermore National Laboratory, 2004.

[26] _ Weighted Essentially Non-Oscillatory Simulations and Modeling of Complex Hydrodynamic Flows. Part 4. Multi-Mode Richtmyer-Meshkov Instability with Reshock, Tech. report, Lawrence Livermore National Laboratory, 2004.

[27] R. J. Leveque, Finite Volume Methods for Hyperbolic Problems, Cambridge University Press, New York, 2002.

[28] H. W. Liepmann and A. Roshko, Elements of Gasdynamics, Galcit aeronautical series, Dover, New York, 2002.

[29] X.-D. Liu, S. Osher, and T. Chan, Weighted essentially non-oscillatory schemes, J. Comput. Phys. 115 (1994), 200-212.

[30] Abd-El-Fattah A. M. and L. F. Henderson, Shock waves at a slow fast gas interface, J. Fluid Mech. 89 (1978), 79-95.

[31] A. Marquina and P. Mulet, A flux-split algorithm applied to conservative models for multicomponent compressible flows, J. Comput. Phys 185 (2003), 120-138.

[32] H. Polachek and R. J. Seeger, On shock-wave phenomena; refraction of shock waves at a gaseous interface, Phys. Rev. 84 (1951), 922-929.

[33] R. Samtaney and D. I. Pullin, Self-similar hypervelocity shock interactions with oblique contact discontinuities, Shock Waves 8 (1998), 299-310.

[34] R. Samtaney and N. J. Zabusky, On shock polar analysis and analytical expressions for vorticity deposition in shock-accelerated density-stratified interfaces, Phys. Fluids 5 (1993), $1285-1287$. 
[35] _ Circulation deposition on shock-accelerated planar and curved density stratified interfaces: models and scaling laws, J. Fluid Mech. 269 (1994), 45-78.

[36] C.-W Shu, High-order finite difference and finite volume WENO schemes and discontinuous Galerkin methods for CFD, Int. J. Comput. Fluid Dyn. 17 (2003), 107-118.

[37] C.-W Shu and S. Osher, Efficient implementation of essentially nonoscillatory shockcapturing schemes, J. Comput. Phys. 77 (1988), 439-471.

[38] _ Efficient implementation of essentially nonoscillatory shock-capturing schemes II, J. Comput. Phys. 88 (1989), 32-78.

[39] A. H. Taub, Refraction of plane shock waves, Phys. Rev. 72 (1947), 51-60.

[40] P. A. Thompson, Compressible Fluid Dynamics, McGraw-Hill Inc., New York, 1972. 